Maria do Socorro Martins Sampaio

\title{
O MÉTODO DE GALERKIN DESCONTÍNUO APLICADO NA INVESTIGAÇÃO DE UM PROBLEMA DE ELASTICIDADE ANISOTRÓPICA
}

Dissertação apresentada à Escola de Engenharia de São Carlos da Universidade de São Paulo, como parte dos requisitos para a obtenção do título de Mestre em Engenharia de Estruturas.

Orientador: Prof. Ph.D. Adair Roberto Aguiar

São Carlos

2009 
AUTORIZO A REPRODUÇÃO E DIVULGAÇĀO TOTAL OU PARCIAL DESTE TRABALHO, POR QUALQUER MEIO CONVENCIONAL OU ELETRÔNICO, PARA FINS DE ESTUDO E PESQUISA, DESDE QUE CITADA A FONTE.

Ficha catalográfica preparada pela Seção de Tratamento da Informação do Serviço de Biblioteca - EESC/USP

\begin{tabular}{|c|c|}
\hline \multirow[t]{3}{*}{$5192 \mathrm{~m}$} & $\begin{array}{l}\text { Sampaio, Maria do Socorro Martins } \\
\text { O método de Galerkin descontínuo aplicado na } \\
\text { investigação de um problema de elasticidade } \\
\text { anisotrópica / Marla do Socorro Martins Sampaio ; } \\
\text { orientador Adair Roberto Aguiar. - Sào Carlos, } 2009 \text {. }\end{array}$ \\
\hline & $\begin{array}{l}\text { Dissertaçăo (Mestrado-Programa de Pós-Graduaçăo e Area } \\
\text { de Concentraçăo en Engenharia de Estruturas - Escola de } \\
\text { Engenharia de Săo Carlos da Universidade de Săo Paulo, } \\
2009 \text {. }\end{array}$ \\
\hline & $\begin{array}{l}\text { 1. Elasticidade anisotrópica. 2. Problema de } \\
\text { equilibrio. 3. Método dos elementos finitos. 4. Método } \\
\text { de Galerkin descontinuo. I. Título. }\end{array}$ \\
\hline
\end{tabular}


FOLHA DE JULGAMENTO

Candidata: Engenheira MARIA DO SOCORRO MARTINS SAMPAIO

Dissertação defendida e julgada em 08/07/2009 perante a Comissão Julgadora:

Adair R. Aquiar $\quad$ Aprovado

P.of. Dr. ADAIR ROBERTO AGUIAR (Orientador)

(Escola de Engenharia de São Carlos/USP)

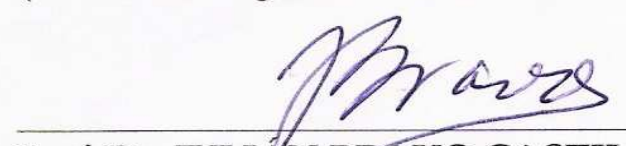

Aprovado

Prof. Dr. JULIANN BRAVO CASTILLERO (Universidade La Havana)

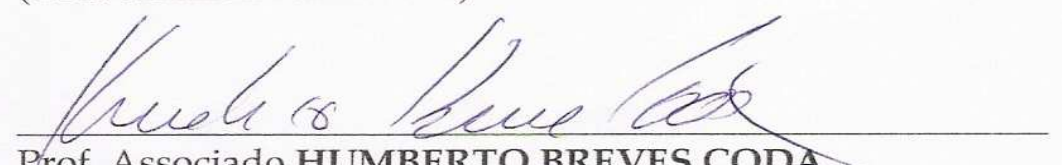

APROUACO

(Escola de Engenharia de São Carlos/USP)

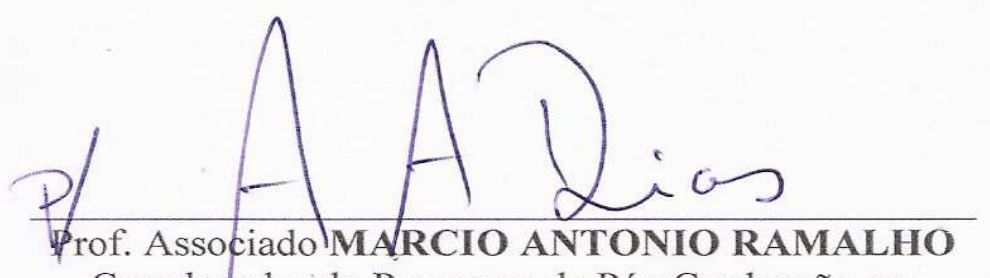

Coordenador do Programa de Pós-Graduação em Engenharia Civil (Engenharia de Estruturas)

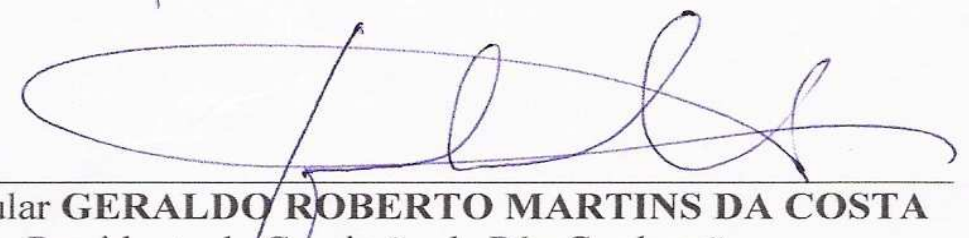

Presidente da Comissão de Pós-Graduação 

Aos meus avós paternos:

Geraldo Telesforo Sampaio (in memoriam)

Maria Lêda Teixeira de Sampaio

Aos meus avós maternos:

Francisco Pereira Martins Maria de Oliveira Martins (in memoriam)

Aos meus pais:

Antônio Carlos T. Sampaio

Izabel Martins Sampaio

Aos meus irmãos:

Diego Martins Sampaio Lorena Martins Sampaio

À minha família de coração:

Heinar de Souza Oliveira Maria de Souza Oliveira

Com todo o meu amor. 



\section{AGRADECIMENTOS}

Ao professor Ph. D. Adair Roberto Aguiar pela orientação e dedicação prestadas para o desenvolvimento desta pesquisa.

Aos professores e funcionários do Departamento de Engenharia de Estruturas (SET/EESC/USP). Em especial, à Rosi Aparecida Jordão Rodrigues e ao professor Wilson Sérgio Venturini.

Aos funcionários do Serviço de Biblioteca (EESC/USP) pelos excelentes serviços prestados e em especial à Adriana Coscia Perez Gomes que prontamente providenciou os artigos necessários para o desenvolvimento desta pesquisa.

Ao Conselho Nacional de Desenvolvimento Científico e Tecnológico (CNPq) pela bolsa de estudos concedida.

À Fundação de Amparo à Pesquisa do Estado de São Paulo (FAPESP) pela bolsa de estudos concedida.

Aos professores da Universidade Federal do Amazonas (UFAM) Raimundo Pereira de Vasconcelos pelos anos de orientação e Vilar Fiuza da Camara Júnior pelos muitos ensinamentos transmitidos.

Aos amigos do Departamento de Engenharia de Estruturas: Aref Kzan, Dorival Neto, Edmar Borges, Jesús Sánchez, Raimundo Amorim, Rodrigo Barros, Rodrigo Couto e Wesley Góis. Em especial aos grandes amigos: Fabio Rocha, Jesús Daniel, Isis Lima e Ieda Soraia.

Aos membros da banca, Professor Julián Bravo Castillero (Universidade de Havana/ Cuba) e Professor Humberto Breves Coda (EESC/USP) pelas valiosas sugestões e contribuições realizadas. 

(...)

"Ninguém se engane a si mesmo: se alguém dentre vós se tem por sábio neste mundo, faça-se louco para ser sábio. Porque a sabedoria deste mundo é loucura diante de Deus;

Portanto, ninguém se glorie nos homens; porque tudo é vosso;

Seja Paulo, seja Apolo, seja Cefas, seja o mundo, seja a vida, seja a morte, seja o presente, seja o futuro; tudo é vosso, e vós de Cristo, e Cristo de Deus”.

I Cor 3, 18-23. 



\section{RESUMO}

SAMPAIO, M. S. M. O Método de Galerkin Descontínuo Aplicado na Investigação de um Problema de Elasticidade Anisotrópica. 2009. 134 f. Dissertação (Mestrado) - Escola de Engenharia de São Carlos, Universidade de São Paulo, São Carlos, 2009.

Estuda-se o problema de equilíbrio sem força de corpo de uma esfera anisotrópica sob compressão radial uniformemente distribuída sobre o seu contorno no contexto da teoria da elasticidade linear clássica. A solução deste problema prediz o fenômeno inaceitável da autointersecção em uma região próxima ao centro da esfera para uma dada faixa de parâmetros materiais. Sob o contexto de uma teoria de minimização do funcional de energia potencial total da elasticidade linear clássica com a restrição de que o determinante do gradiente da função mudança de configuração seja injetivo, este fenômeno é eliminado. Aplicam-se duas formulações do Método dos Elementos Finitos de Galerkin Descontínuo (MEFGD) para obter soluções aproximadas para o problema de equilíbrio da esfera sem restrição. A primeira formulação do MEFGD aproxima diretamente os campos de deslocamento e deformação infinitesimal. A consideração do campo adicional de deformação na formulação do MEFGD aumenta o número de graus de liberdade associados aos nós da malha de elementos finitos e, consequentemente, o custo computacional. Com o objetivo de reduzir o número de graus de liberdade, introduz-se neste trabalho uma formulação alternativa do MEFGD. Nesta formulação, o campo de deformação infinitesimal não é obtido diretamente da inversão do sistema de equações resultante, mas sim por pós-processamento, a partir do campo de deslocamento aproximado. As soluções aproximadas obtidas com ambas as formulações do MEFGD são comparadas com a solução exata do problema sem restrição e com soluções aproximadas obtidas com o Método dos Elementos Finitos de Galerkin Clássico (MEFGC). Ambas as formulações do MEFGD fornecem melhores aproximações para a solução exata do que as aproximações obtidas com o MEFGC. Os erros entre a solução exata e as soluções aproximadas obtidas com a formulação alternativa do MEFGD são um pouco maiores do que os erros correspondentes obtidos com a formulação original do MEFGD. Este aumento nos erros é compensado pelo menor esforço computacional exigido pela formulação alternativa. Este trabalho serve de base para o estudo de problemas com restrição de injetividade utilizando o Método de Galerkin Descontínuo.

Palavras-chave: Elasticidade Anisotrópica, Problema de Equilíbrio, Método dos Elementos Finitos, Método de Galerkin Descontínuo. 



\section{ABSTRACT}

SAMPAIO, M. S. M. The Discontinuous Galerkin Method Applied to the Investigation of an Anisotropic Elasticity Problem. 2009. 134 f. Dissertation (M.Sc) - Escola de Engenharia de São Carlos, Universidade de São Paulo, São Carlos, 2009.

The equilibrium problem without body force of an anisotropic sphere under radial compression that is uniformly distributed on the sphere's boundary is investigated in the context of the classical linear elasticity theory. The solution of this problem predicts the unacceptable phenomenon of self-intersection in a vicinity of the center of the sphere for a given range of material parameters. This phenomenon can be eliminated in the context of a theory that minimizes the total potential energy of classical linear elasticity subjected to the restriction that the deformation field be injective. Two formulations of the Finite Element Method using Discontinuous Galerkin (MEFGD) are used to obtain approximate solutions for the unconstrained problem. The first formulation of the MEFGD approximates both the displacement and the strain fields. The consideration of the strain as an additional field in the formulation of the MEFGD increases the number of degrees of freedom associated to the finite elements and, therefore, the computational cost. With the objective of reducing the number of degrees of freedom, an alternative formulation of the MEFGD is introduced in this work. In this formulation, the strain field is not obtained directly from the inversion of the resulting linear system of equations, but from a post-processing calculation using the approximate displacement field. The approximate solutions obtained with both formulations of the MEFGD are compared with the exact solution of the problem without restriction and with approximate solutions obtained with the Finite Element Method using Classical Galerkin (MEFGC). Both formulations of the MEFGD yield better approximations for the exact solution than the approximations obtained with the MEFGC. The errors between the exact solution and the approximate solutions obtained with the alternative formulation of the MEFGD are slightly higher than the corresponding errors obtained with the original formulation of the MEFGD. These errors are compensated by the fact that the alternative formulation requires less computational effort than the computational effort required by the original formulation. This work serves as a basis for the study of problems with the injectivity restriction using the Discontinuous Galerkin Method.

Keywords: Anisotropic Elasticity, Equilibrium Problem, Finite Element Method, Discontinuous Galerkin Method. 



\section{LISTA DE FIGURAS}

Figura 2.1. Problema do disco sob compressão radial uniformemente distribuída ...................30

Figura 2.2. Problema do bloco colado, adaptado de Aguiar (2000) ........................................ 31

Figura 2.3. Problema do punção colado, adaptado de Aguiar e Fosdick (2001) ...................... 32

Figura 2.4. Problema do disco girando com velocidade angular constante e sob pressão radial

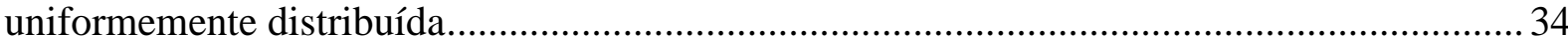

Figura 2.5. Problema do tubo sob pressão radial uniformemente distribuída ........................... 35

Figura 2.6. Simulação numérica mostrando o fenômeno da auto-intersecção, adaptado de

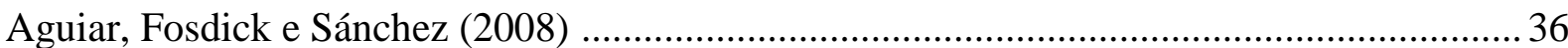

Figura 2.7. Problema estudado por Duarte, Rochinha e Carmo (2000) ................................... 40

Figura 3.1. Ponto no espaço em coordenadas esféricas........................................................ 47

Figura 3.2. Corpo seccionado sob a ação de forças internas e externas ................................... 48

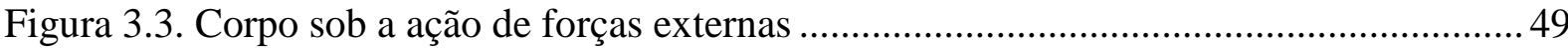

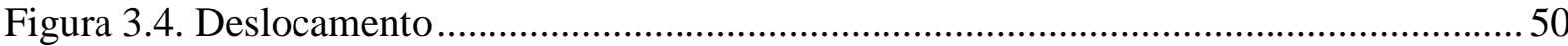

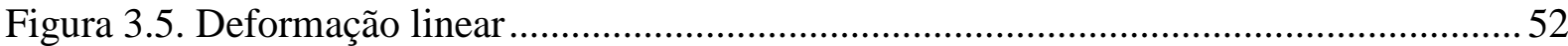

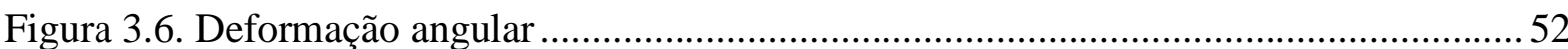

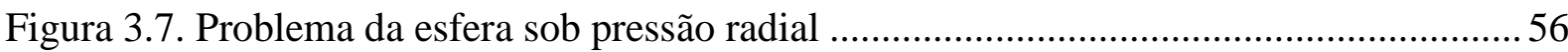

Figura 3.8. Configuração de referência e atual de um corpo.................................................... 63

Figura 5.1. Problema da esfera com regiões onde a restrição da injetividade está ativa, ou,

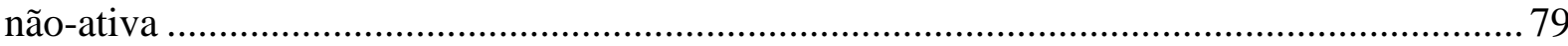

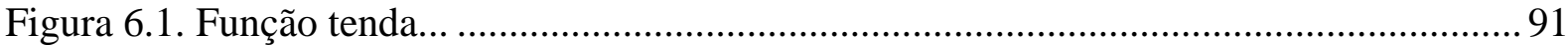

Figura 6.2. Graus de liberdade dos elementos intermediários na formulação original do MEFGD 98

Figura 6.3. Graus de liberdade do elemento 1 na formulação original do MEFGD.. 99

Figura 6.4. Graus de liberdade do elemento n na formulação original do MEFGD. 99

Figura 6.5. Graus de liberdade dos elementos intermediários na formulação alternativa do MEFGD 103

Figura 6.6. Graus de liberdade do elemento 1 na formulação alternativa do MEFGD .......... 103

Figura 6.7. Graus de liberdade do elemento n na formulação alternativa do MEFGD .......... 104

Figura 7.1. Solução exata u x solução $u_{h}$ com MEFGC para diferentes malhas .................... 108 
Figura 7.2. Solução exata u' x solução $\mathrm{q}_{\mathrm{h}}$ com MEFGC para diferentes malhas 109

Figura 7.3. Solução exata u x solução $u_{h}$ com MEFGD original para diferentes malhas..... 110

Figura 7.4. Solução exata u' x solução $q_{h}$ com MEFGD original para diferentes malhas......111

Figura 7.5. Solução exata $u$ x solução $u_{h}$ com MEFGD alternativo para diferentes malhas ..112

Figura 7.6. Solução exata u' x solução qh com MEFGD alternativo para diferentes malhas. 113

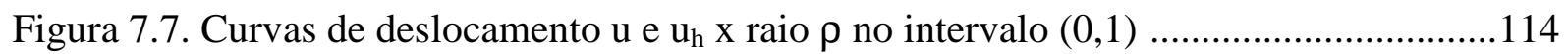

Figura 7.8. Curvas de deformação u' e q $\mathrm{x}$ raio $\rho$ no intervalo $(0,1)$

Figura 7.9. Curvas de erro obtidas de resultados gerados pelo MEFGC, MEFGD original e MEFGD alternativo 116

Figura 7.10. Curvas de erro obtidas de resultados gerados pelo MEFGC, MEFGD original e MEFGD alternativo 118

Figura 7.11. Curvas de convergência monótona e não monótona (Fonte: Soriano, 2003) .....119

Figura 7.12. Curvas deslocamento $x \log _{2} \mathrm{n}$ para ponto na região sem auto-intersecção ..........120

Figura 7.13. Curvas deslocamento $\mathrm{x} \log _{2} \mathrm{n}$ para ponto na região com auto-intersecção..........121

Figura 7.14. Curvas de deslocamento obtidas com MEFGD original e MEFGD alternativo 121

Figura 7.15. Curvas deformação $x \log _{2} \mathrm{n}$ para ponto na região sem auto-intersecção

Figura 7.16. Curvas deformação $\mathrm{x} \log _{2} \mathrm{n}$ ampliadas obtidas com MEFGD original e MEFGD alternativo 123

Figura 7.17. Curvas deformação $x \log _{2} \mathrm{n}$ para ponto na região com auto-intersecção 123

Figura 7.18. Curvas deformação x $\log _{2} \mathrm{n}$ ampliadas obtidas com MEFGD original e MEFGD alternativo. 


\section{SUMÁRIO}

CAPÍTULO 1 - INTRODUÇÃ̃O ....................................................................................... 21

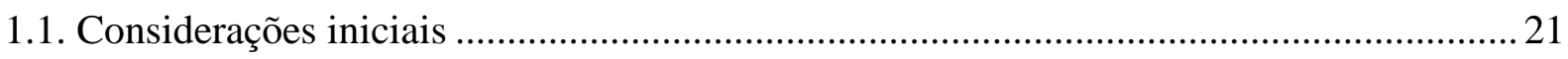

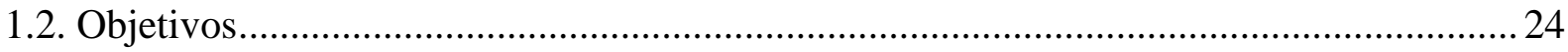

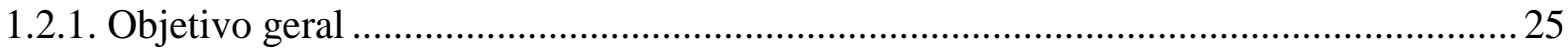

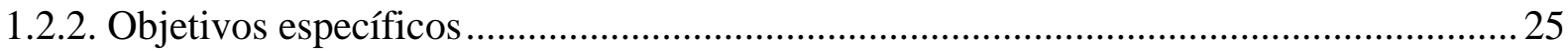

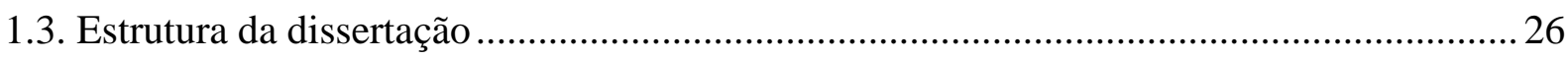

CAPÍTULO 2 - REVISÃO BIBLIOGRÁFICA .........................................................29

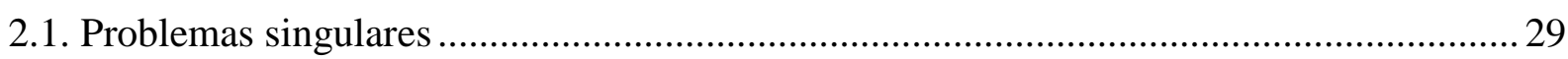

2.2. Método dos Elementos Finitos de Galerkin Descontínuo ................................................. 36

CAPÍTULO 3 - TEORIA DA ELASTICIDADE LINEAR CLÁSSICA ............................ 45

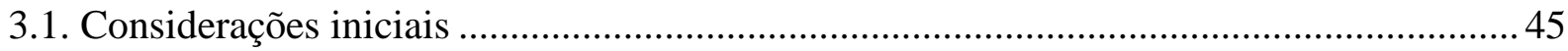

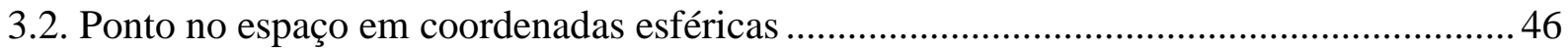

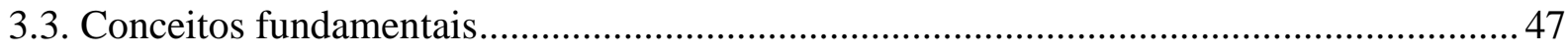

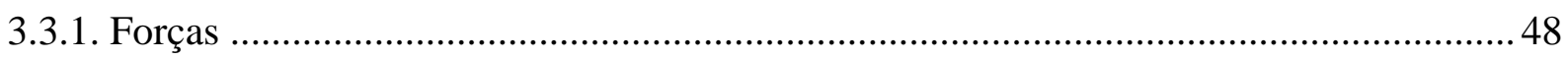

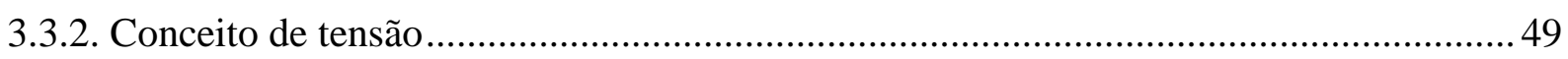

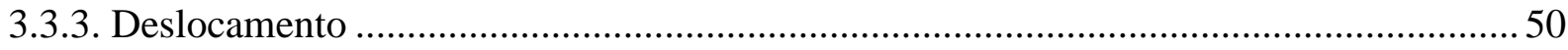

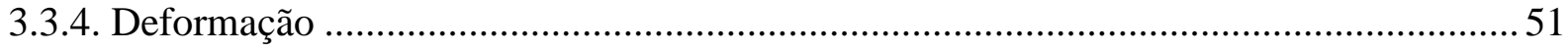

3.4. Equações básicas da teoria da elasticidade linear clássica .............................................53

3.4.1. Equações de equilíbrio em coordenadas esféricas.......................................................53

3.4.2. Relações deformação-deslocamento em coordenadas esféricas....................................54

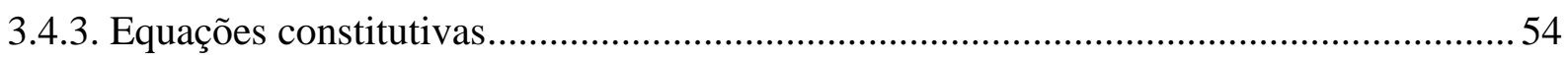

3.5. A teoria da elasticidade linear clássica aplicada ao problema da esfera ......................... 55

3.6. Obtenção da solução exata do problema da esfera .......................................................... 59

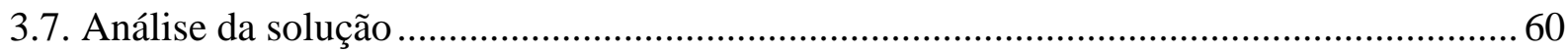

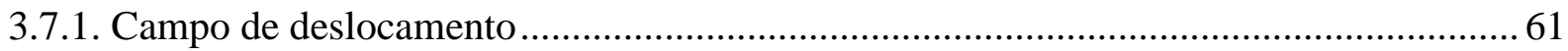

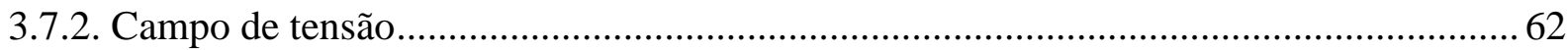

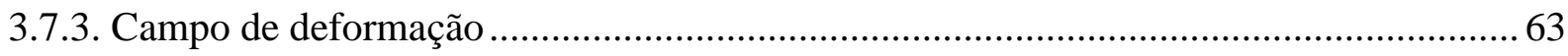




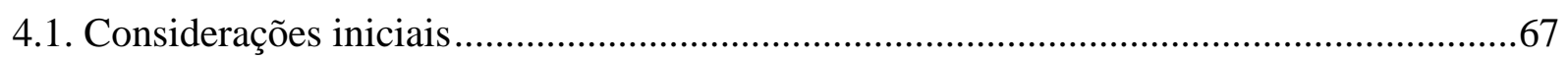

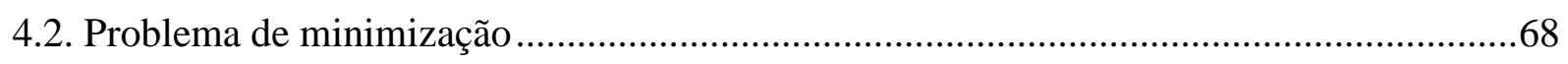

4.3. Princípio da mínima energia potencial.......................................................................68

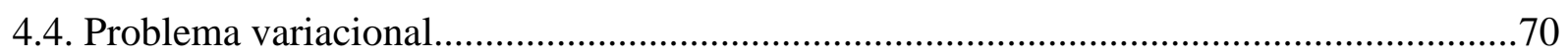

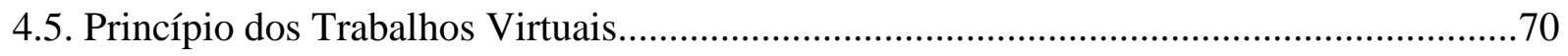

4.6. Energia de deformação da esfera elástica anisotrópica..................................................71

4.7. Energia potencial das forças externas que atuam na esfera elástica anisotrópica..............72

CAPÍTULO 5 - O PROBLEMA DE MINIMIZAÇÃO COM RESTRIÇÃO.....................75

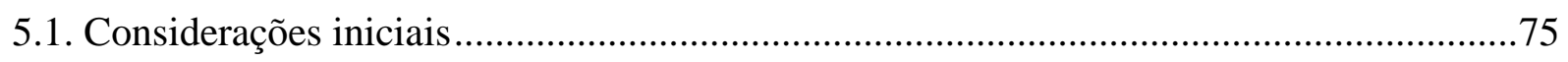

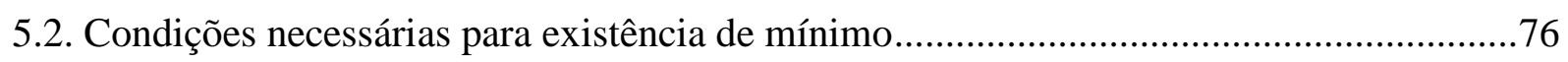

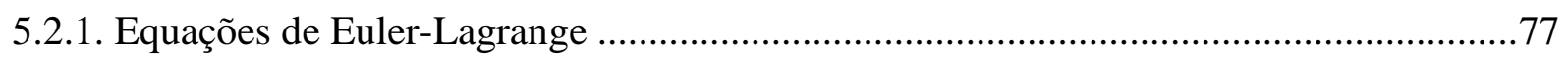

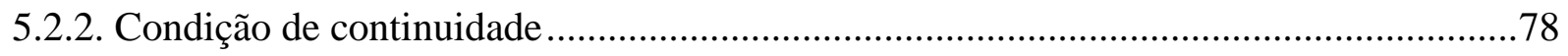

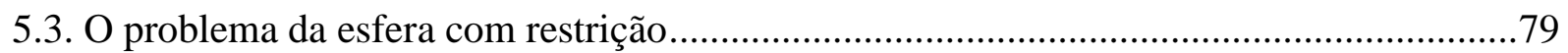

CAPÍTULO 6 - SOLUÇÃO DISCRETA DO PROBLEMA DA ESFERA OBTIDA VIA

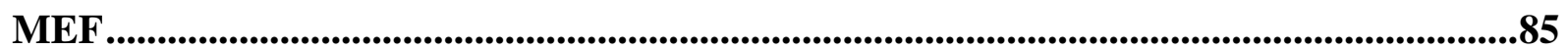

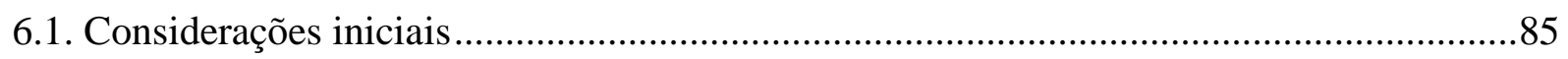

6.2. Características gerais do método dos elementos finitos de Galerkin clássico ....................86

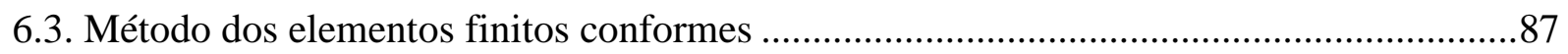

6.4. Método dos elementos finitos de Galerkin descontínuo ....................................................88

6.5. Formulação discreta para o problema da esfera sem restrição utilizando-se o MEFGC ...89

6.6. Formulação discreta para o problema da esfera sem restrição utilizando-se o MEFGD ..93

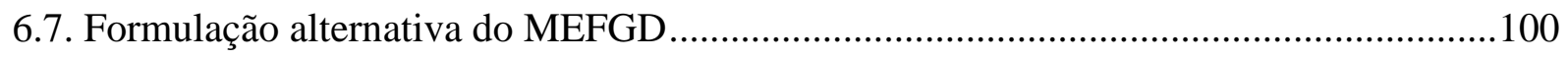

CAPÍTULO 7 - RESULTADOS ..............................................................................107

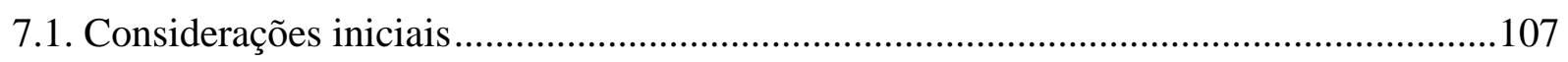

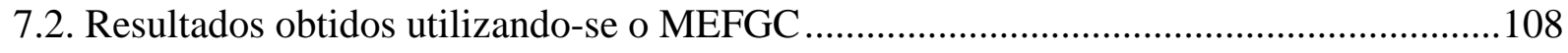

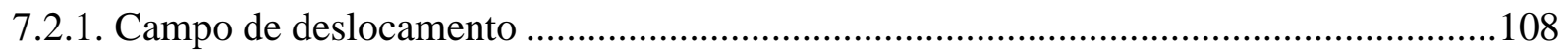

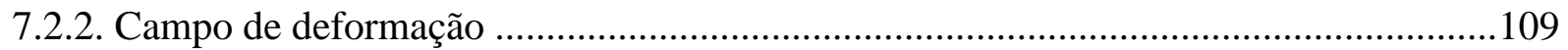

7.3. Resultados obtidos com o MEFGD - Formulação original ..............................................110

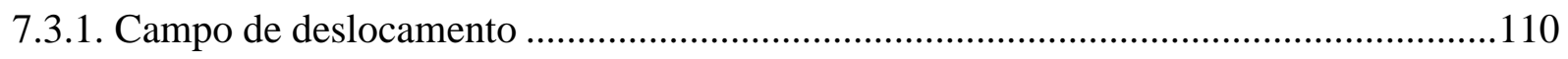

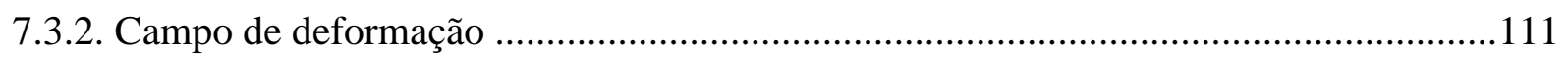

7.4. Resultados obtidos com o MEFGD - Formulação alternativa ......................................112

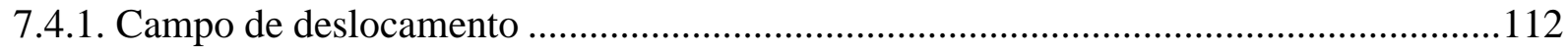

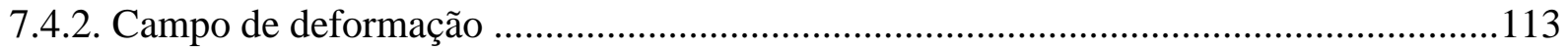


7.5. Comparação dos Resultados

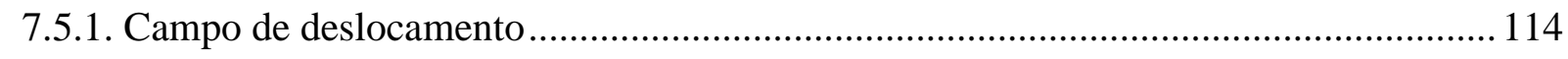

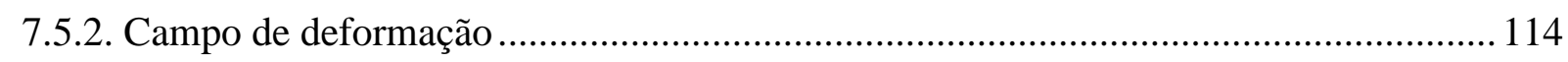

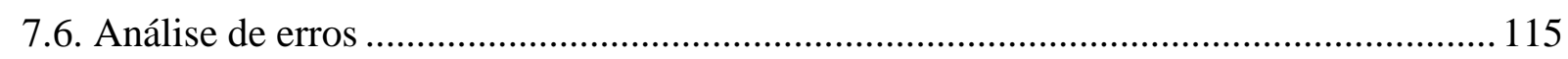

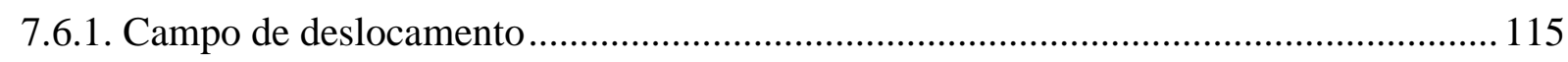

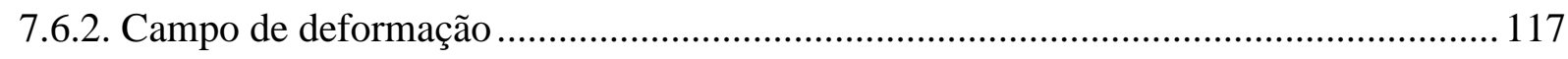

7.7. Análise de convergência dos métodos em pontos situados dentro e fora da região de auto-

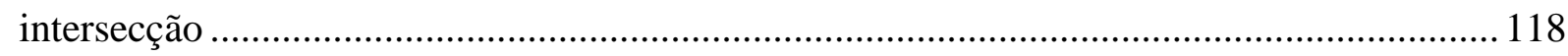

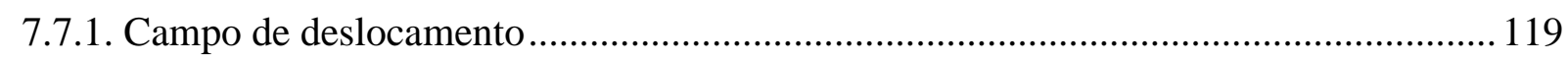

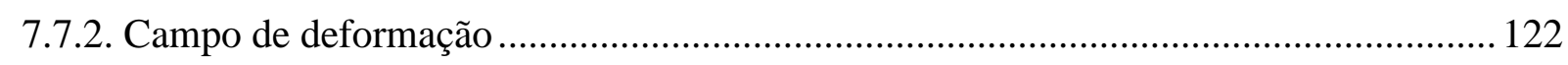

CAPÍTULO 8 - CONCLUSÕES ..................................................................................... 125

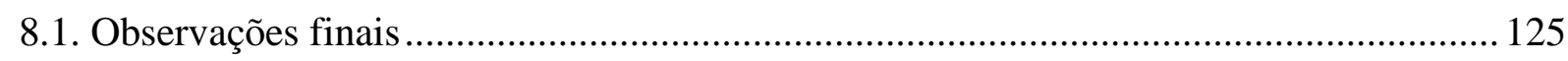

REFERÊNCIAS BIBLIOGRÁFICAS ............................................................................... 129 



\subsection{CONSIDERAÇÕES INICIAIS}

Estuda-se o problema de equilíbrio de uma esfera anisotrópica sob compressão radial uniformemente distribuída no contexto da teoria da elasticidade linear clássica. A solução deste problema prediz o fenômeno anômalo da auto-intersecção em uma região próxima ao centro da esfera. Este fenômeno é eliminado no contexto de uma teoria de minimização com restrição. Neste trabalho, aplica-se o Método dos Elementos Finitos de Galerkin Descontínuo (MEFGD) para obter soluções aproximadas para o problema de equilíbrio sem restrição.

O comportamento de um corpo em equilíbrio, para uma dada condição de carregamento externo e vinculação no contorno, fica completamente determinado conhecendo-se, em qualquer um de seus pontos, os campos de deslocamento, tensão e deformação (ODEN, 1967; PROENÇA, 2007). Os modelos matemáticos construídos para a determinação destes campos são governados por equações diferenciais parciais elípticas, cujas soluções são, em geral, suaves em todos os pontos interiores do corpo (DE GIORGI, 1968; FOSDICK; FREDDI; ROYER-CARFAGNI, 2007; NASH, 1958; WEINBERGER, 1965).

Embora a Teoria da Elasticidade Linear Clássica aproxime e prediga de forma 
satisfatória o comportamento de tais corpos, as soluções de alguns problemas governados por estas equações diferenciais podem, no entanto, apresentar singularidades que se manifestam de duas formas distintas. Na primeira forma, o gradiente de deslocamento tende ao infinito à medida que se aproxima de vértices de trincas, determinados pontos interiores e cantos no contorno de sólidos (AGUIAR; FOSDICK, 2001; ARAVAS; SHARMA, 1991; ATKINSON, 1977; COMNINOU, 1977; ENGLAND， 1965; KNOWLES，1981; WILLIAMS， 1959; MUSKHELISHVILI, 1933; etc.). A outra forma está associada à violação da condição cinemática de que o determinante do gradiente da função mudança de configuração é positivo em todos os pontos do sólido, caracterizando o fenômeno da auto-intersecção da matéria (AGUIAR, 2006b; FOSDICK; ROYER-CARFAGNI, 2001). Ambas as formas de singularidades são inaceitáveis do ponto de vista físico.

O que se observa nos problemas que apresentam os tipos de singularidades descritos acima é que as deformações não são infinitesimais, ou seja, em torno dos pontos singulares as deformações excedem os níveis aceitáveis do ponto de vista da Teoria da Elasticidade Linear Clássica e, em geral, o mapeamento deixa de ser injetivo.

Um possível caminho para preservar a injetividade consiste em assumir adequadamente propriedades elásticas não-lineares para descrever o comportamento do material na vizinhança destes pontos singulares (AGUIAR; FOSDICK, 2001).

Outra forma de tratar o fenômeno anômalo da auto-intersecção é apresentada por Fosdick e Royer-Carfagni (2001). Estes autores propõem minimizar o funcional de energia potencial total da elasticidade linear clássica submetido à restrição da injetividade e encontram as condições necessárias para a existência de mínimo do problema assim formulado. A restrição cinemática da injetividade é uma hipótese constitutiva (GURTIN, 1981). Os autores aplicam as condições necessárias obtidas para resolver o problema do disco de Lekhnitskii (1968) e verificam que o fenômeno anômalo da auto-intersecção da matéria é 
eliminado.

Aguiar (2006b) utiliza a teoria proposta por Fosdick e Royer-Carfagni (2001) para investigar o problema da esfera de Ting (1999) e encontra uma solução fechada que elimina a auto-intersecção da matéria predita pela solução obtida utilizando-se a teoria da elasticidade linear clássica. Como o problema de minimização com restrição oriundo da aplicação da teoria de Fosdick e Royer-Carfagni (2001) na investigação de problemas de elasticidade é altamente não-linear, em muitos casos é necessário utilizar uma ferramenta numérica para obter soluções aproximadas para o problema. Aguiar (2006b) propõe, então, uma formulação de penalidades juntamente com o método dos elementos finitos utilizando funções contínuas e lineares por partes para investigar numericamente o problema da esfera e obtém boas soluções aproximadas para o problema em relação aos resultados analíticos correspondentes.

Com base no exposto acima, estuda-se o problema da esfera de Ting (1999) no contexto da teoria da elasticidade linear clássica e da teoria de minimização com restrição proposta por Fosdick e Royer-Carfagni (2001). Aplica-se o MEFGD para se obter soluções aproximadas para o problema de equilíbrio sem restrição.

O MEFGD é um método para resolução aproximada de equações diferenciais, no qual as funções aproximativas e ponderadoras adotadas são descontínuas entre elementos adjacentes, sendo, portanto, diferente do Método dos Elementos Finitos de Galerkin Clássico (MEFGC) em que se assume a continuidade destas funções admissíveis. O MEFGD foi introduzido em 1973 por Reed e Hill para a resolução numérica de sistemas hiperbólicos de primeira ordem. No mesmo período, mas de forma independente, o método foi introduzido como um esquema não-convencional para a resolução aproximada de equações diferenciais parciais elípticas de segunda ordem (COCKBURN; KARNIADAKIS; SHU, 2000).

A principal vantagem do MEFGD é que ele permite tratar problemas cujas soluções apresentam descontinuidades no interior do domínio em que estão definidas, sem deteriorar a 
qualidade da solução na vizinhança da região onde ocorrem estas descontinuidades. Outro atrativo do método para problemas de elasticidade é que ele é localmente conservativo, garantindo que todos os elementos estão em equilíbrio, mesmo aqueles próximos a singularidades (FORTI, 2005).

Neste trabalho, empregam-se duas formulações do MEFGD para obter soluções aproximadas para o problema de equilíbrio da esfera sem restrição. A primeira formulação do MEFGD baseia-se no procedimento utilizado por Castillo (2003) e consiste em aproximar diretamente os campos de deslocamento e de deformação infinitesimal da esfera. A consideração do campo adicional de deformação infinitesimal na formulação variacional do MEFGD aumenta o número de graus de liberdade associados aos nós da malha de elementos finitos utilizada para discretizar o domínio e, consequentemente, o custo computacional. Com o objetivo de reduzir o número de graus de liberdade, introduz-se uma formulação alternativa do MEFGD, onde o campo de deformação infinitesimal não é obtido diretamente da inversão do sistema de equações lineares resultante, mas por pós-processamento, a partir do campo de deslocamento.

Os resultados numéricos obtidos para o problema de equilíbrio da esfera anisotrópica sob compressão radial uniformemente distribuída sem restrição utilizando-se o MEFGD são comparados com a solução analítica do problema e com resultados numéricos obtidos utilizando-se o MEFGC. Análises de erros e de convergência das soluções aproximadas são realizadas.

\subsection{OBJETIVOS}

Estudar e aplicar uma formulação do Método dos Elementos Finitos de Galerkin Descontínuo (MEFGD) na resolução de um problema de elasticidade anisotrópica de grande 
interesse na Mecânica Aplicada.

A pesquisa tem por base os trabalhos de Ting (1999) e de Aguiar (2006b), que estudam o problema da esfera anisotrópica sob compressão radial uniformemente distribuída, e de Castillo (2003), que apresenta uma formulação do MEFGD para a resolução de equações diferencias parciais elípticas.

\subsubsection{OBJETIVO GERAL}

Estudar o problema da esfera anisotrópica sob compressão radial uniformemente distribuída no contexto da teoria da elasticidade linear clássica, cuja solução prediz o fenômeno da auto-intersecção em uma região próxima ao centro da esfera, e no contexto de uma teoria de minimização com restrição, que elimina este fenômeno. Aplicar o MEFGD para obter soluções aproximadas para o problema de equilíbrio sem restrição.

\subsubsection{OBJETIVOS ESPECÍFICOS}

Calcular o campo de deslocamento aproximado da esfera anisotrópica sob compressão radial uniformemente distribuída utilizando-se o MEFGC e o MEFGD. Comparar os resultados obtidos numericamente utilizando-se o MEFGD com a solução exata do problema e com os resultados numéricos obtidos utilizando-se o MEFGC.

Gerar sequiências de campos aproximados parametrizados pelo tamanho característico da malha de elementos finitos e verificar os erros obtidos à medida que o tamanho característico da malha tende a zero.

Analisar a convergência das soluções aproximadas obtidas com ambos os métodos à medida que se refina a malha de elementos finitos. 


\subsection{ESTRUTURA DA DISSERTAÇÃO}

No Capítulo 2 apresenta-se a revisão bibliográfica relacionada ao estudo do fenômeno anômalo da auto-intersecção e ao estudo do MEFGD aplicado na busca de soluções aproximadas de equações diferenciais elípticas, ou, de equações que contenham operadores elípticos em sua estrutura.

No Capítulo 3 descrevem-se alguns conceitos da Teoria da Elasticidade Linear Clássica e apresenta-se a solução do problema da esfera anisotrópica sob compressão radial uniformemente distribuída e a caracterização do fenômeno da auto-intersecção da matéria predito por esta solução.

No Capítulo 4 descrevem-se o Princípio dos Trabalhos Virtuais (PTV) e o Método da Energia que permitem obter diretamente a formulação variacional fraca de um problema sem a necessidade de se conhecer previamente a forma forte deste problema.

No Capítulo 5 apresenta-se a teoria de minimização com restrição proposta por Fosdick e Royer-Carfagni (2001) e a sua a aplicação ao problema da esfera.

No Capítulo 6 apresentam-se alguns fundamentos do MEFGC utilizados para obter soluções aproximadas para o problema da esfera e as duas formulações do MEFGD estudadas.

No Capítulo 7 apresentam-se as soluções numéricas obtidas para o problema da esfera utilizando-se as formulações apresentadas no Capítulo 6. Os resultados obtidos utilizando-se as formulações apresentadas são comparados à solução exata do problema apresentada no Capítulo 3 e com resultados numéricos obtidos utilizando-se o MEFGC. Curvas de erros entre as soluções aproximadas e a solução exata e curvas de convergência das soluções aproximadas são apresentadas.

No Capítulo 8 relacionam-se algumas conclusões realizadas a partir dos resultados 
obtidos com o desenvolvimento do trabalho.

Por fim, listam-se, em ordem alfabética, as referências bibliográficas consultadas durante o desenvolvimento deste trabalho. 


\subsection{PROBLEMAS SINGULARES}

As soluções obtidas com a utilização da teoria da elasticidade linear clássica para problemas de cilindros, esferas, sólidos com cantos, etc., podem apresentar singularidades que se manifestam de duas formas distintas. Na primeira, o gradiente de deslocamento tende ao infinito à medida que se aproxima de vértices de trincas, determinados pontos interiores e cantos no contorno de sólidos. A outra forma está associada à violação da condição cinemática de que o determinante do gradiente da função mudança de configuração é positivo em todos os pontos do sólido. Esta violação caracteriza o fenômeno anômalo da autointersecção da matéria.

Os estudos realizados para identificar tais fenômenos em problemas de elasticidade e propor novas formas de tratamento são numerosos. Sendo assim, buscou-se relacionar alguns dos principais trabalhos desenvolvidos, no sistema autor-data, que melhor descrevem os avanços realizados no estudo dos problemas que apresentam estes tipos de singularidades.

Lekhnitskii (1968) observa que a tensão no centro de um disco homogêneo de raio $\rho_{\mathrm{e}}$ com anisotropia cilíndrica pode ser infinita quando o disco é submetido a uma pressão radial 
p uniformemente distribuída sobre o contorno do disco (Fig. 2.1). Fosdick e Royer-Carfagni (2001) observam que a solução deste problema sem restrição também prediz a autointersecção do material em uma região central do disco.

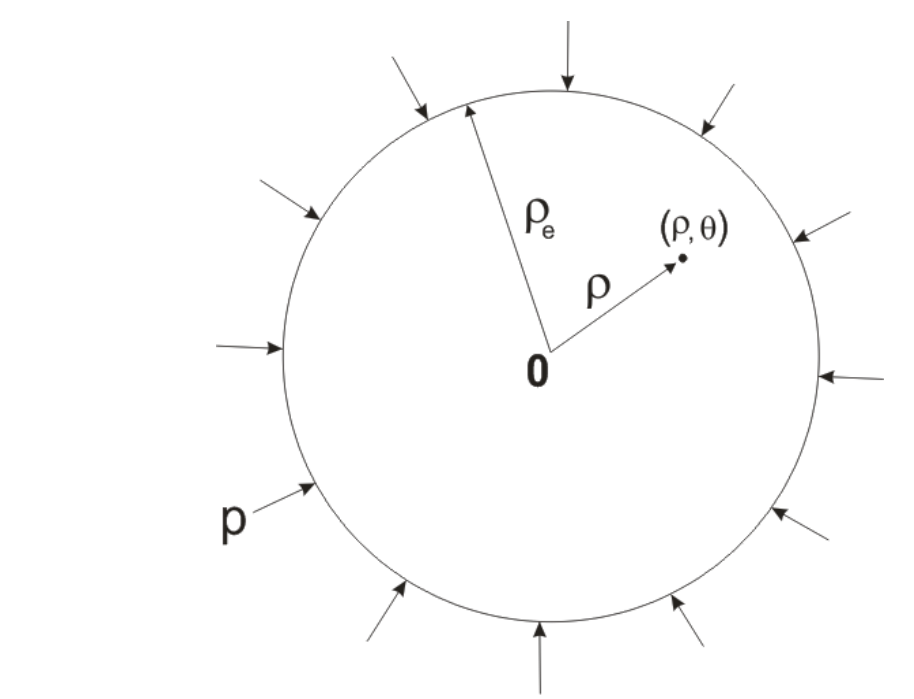

Figura 2.1. Problema do disco sob compressão radial uniformemente distribuída.

Antman e Negron Marrero (1987) estudam uma esfera de material elástico não-linear submetida a uma pressão radial uniformemente distribuída por unidade de área e mostram que quando a pressão aplicada na superfície externa da esfera excede um valor crítico a tensão no centro da esfera é infinita.

Ting (1999) estuda o problema de Antman e Negron Marrero (1987) considerando uma esfera de material elástico linear com anisotropia esférica. Ting também observa a existência de tensão infinita no centro da esfera, porém, diferentemente do problema estudado por Antman e Negron Marrero, no problema de Ting a existência de tensão infinita independe da magnitude da pressão aplicada na superfície externa da esfera, dependendo somente das propriedades elásticas do material.

Aguiar e Fosdick (2000) empregam um método de elementos finitos baseado em uma técnica de integração seletiva e reduzida com penalização para construir soluções numéricas para o problema do bloco colado. O problema consiste em um prisma de seção transversal quadrada e comprimento infinito. A seção transversal do prisma é constituída por duas 
superfícies opostas engastadas, unidas a duas superfícies laterais opostas livres. Impõe-se um deslocamento compressivo $\Delta$ da direita para a esquerda do bloco (Fig. 2.2). Considera-se que o bloco é incompressível. A deformação da seção transversal é descrita pelas funções

$$
\mathrm{x}_{1}=\mathrm{y}_{1}\left(\mathrm{X}_{1}, \mathrm{X}_{2}\right), \mathrm{x}_{2}=\mathrm{y}_{2}\left(\mathrm{X}_{1}, \mathrm{X}_{2}\right),
$$

em relação a uma base ortornormal $\left\{i_{1}, i_{2}\right\}$ com origem no canto inferior esquerdo do bloco. A solução exata para este problema de valor de contorno não-linear não é conhecida. As soluções obtidas por Aguiar e Fosdick (2000) convergem para a solução do problema em todos os pontos, exceto em uma pequena vizinhança do canto do bloco colado.

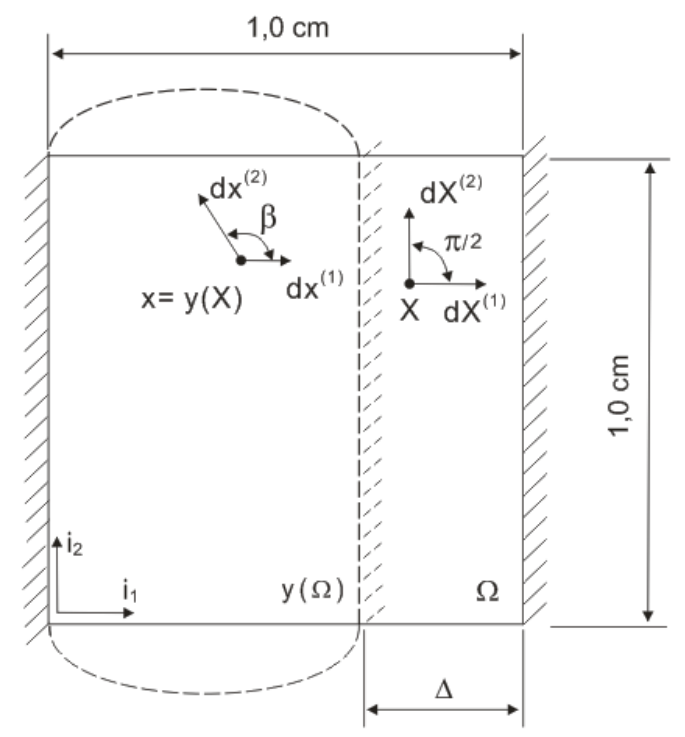

Figura 2.2. Problema do bloco colado, adaptado de Aguiar (2000).

Um importante avanço no tratamento do fenômeno anômalo da auto-intersecção foi alcançado com o trabalho de Fosdick e Royer-Carfagni (2001), que propõem minimizar o funcional quadrático da energia potencial da elasticidade linear clássica impondo a restrição de que o campo de deslocamento é injetivo. Um teorema de existência para minimizadores de problemas planos é apresentado. Os autores aplicam as condições necessárias para existência de mínimo encontradas para resolver o problema do disco de Lekhnitskii (1968) e encontram 
uma solução exata do problema, que não apresenta o fenômeno anômalo da auto-intersecção.

Aguiar e Fosdick (2001) apresentam uma importante contribuição no tratamento da auto-intersecção na vizinhança de cantos de sólidos através do estudo do problema do punção colado. O problema consiste em achar o campo de deslocamento de um meio elástico semiinfinito submetido a uma força de compressão $\mathrm{P}$ por meio de um punção colado sobre uma parte da superfície plana do contorno deste meio elástico (Fig. 2.3). A região do meio semiinfinito próxima aos cantos do punção apresenta comportamento singular. Os autores realizam uma análise assintótica juntamente com uma investigação numérica utilizando o método dos elementos finitos nos contextos das teorias de elasticidade linear e não-linear e verificam que no contexto da teoria não-linear é possível obter um comportamento assintótico que, enquanto ainda singular, previne a auto-intersecção da matéria.

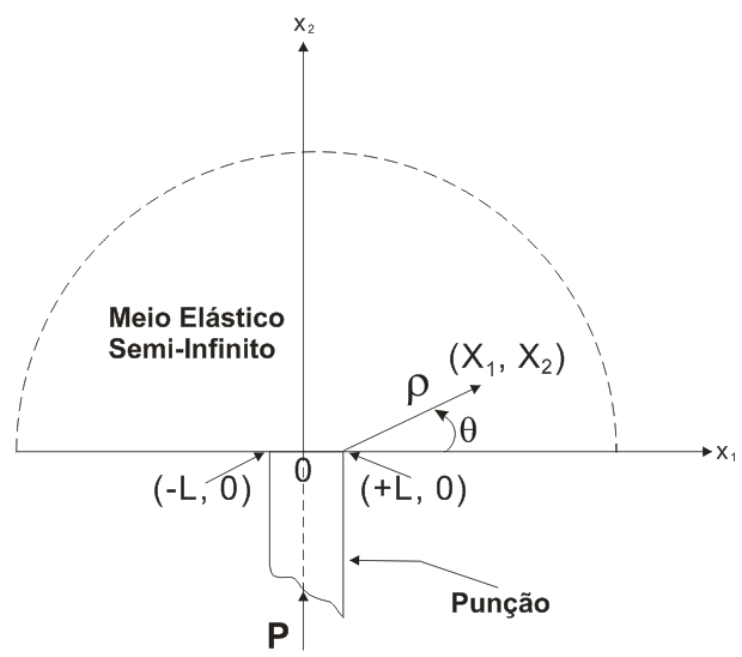

Figura 2.3. Problema do punção colado, adaptado de Aguiar e Fosdick (2001).

Tarn (2002) estuda a singularidade no campo de tensão de um cilindro elástico com anisotropia cilíndrica submetido à pressão radial uniformemente distribuída. $\mathrm{O}$ autor realiza seu estudo partindo do pressuposto que a singularidade no campo de tensão pode ser atribuída a uma definição contraditória de anisotropia no centro do cilindro, e considera que o mesmo é composto por duas regiões, uma externa constituída de material cilindricamente anisotrópico e a outra interna constituída de material transversalmente isotrópico. $\mathrm{O}$ autor obtém a solução 
fechada para o problema. As análises realizadas mostram que os campos de deslocamento e tensão dependem somente das propriedades elásticas do material. O autor observa que quando o raio da região interna, contendo o material transversalmente isotrópico, diminui, a magnitude das tensões no centro do cilindro diminui se $\kappa>1$ e aumentam se $\kappa<1$. Ainda assim, as tensões são finitas e o campo de deslocamento descreve um mapeamento injetivo, levando o autor a concluir que a singularidade em tensões não ocorre se a anisotropia cilíndrica for definida apropriadamente na região central do cilindro.

Aguiar (2004) propõe uma formulação de penalidades interiores para investigar numericamente o problema do disco de Lekhnitskii (1968) no contexto da teoria proposta por Fosdick e Royer-Carfagni (2001). As soluções aproximadas obtidas com a formulação apresentada pelo autor confirmam numericamente a solução analítica encontrada por Fosdick e Royer-Carfagni (2001).

Aguiar e Sánchez (2005) empregam o Método dos Elementos Finitos com refinamento p para resolver numericamente o problema do disco de Lekhnitskii (1968) e obtêm resultados que indicam que a solução numérica converge lentamente para a solução analítica do problema.

Aguiar (2006a) propõe uma formulação de penalidades exteriores para investigar numericamente o problema do disco de Lekhnitskii (1968) sob o ponto de vista da teoria proposta por Fosdick e Royer-Carfagni (2001) e obtém resultados que convergem para a solução analítica do problema.

Aguiar (2006b) utiliza a teoria proposta por Fosdick e Royer-Carfagni (2001) para investigar o problema da esfera de Ting (1999) e encontra uma solução fechada para o problema que elimina a auto-intersecção da matéria predita pela teoria da elasticidade linear clássica. Como o problema de minimização com restrição oriundo da aplicação da teoria de Fosdick e Royer-Carfagni (2001) na investigação de problemas de elasticidade é altamente 
não-linear, em muitos casos é necessário utilizar uma ferramenta numérica para obter soluções aproximadas para o problema. Nesse sentido, Aguiar (2006b) propõe uma formulação de penalidades interiores para investigar numericamente o problema da esfera de Ting. O autor utiliza o método dos elementos finitos com funções contínuas, lineares por partes, para aproximar as soluções do problema de minimização. Esta metodologia fornece boas aproximações para o problema.

Aguiar e Silva (2007) estudam o problema de um disco cilindricamente anisotrópico que gira em torno do seu centro com velocidade angular $\omega$ constante (Fig. 2.4). O disco é comprimido radialmente ao longo do seu contorno externo por uma força normal uniformemente distribuída $\mathrm{p}$. Para o caso particular de velocidade angular nula, $\omega=0$, temse o problema do disco de Lekhnitskii (1968), cuja solução prediz a auto-intersecção da matéria para qualquer valor da força compressiva aplicada no contorno e para um intervalo de valores do parâmetro $\kappa$ que contém as propriedades elásticas do material. Para o disco com velocidade angular não-nula, $\omega \neq 0$, os autores mostram que a auto-intersecção da matéria não ocorre apenas para valores de força compressiva que são pequenos quando comparados com o valor de $\omega^{2}$. Para outros valores de força compressiva a auto-intersecção ocorre e os autores utilizam a teoria de Fosdick e Royer-Carfagni (2001) para eliminar este fenômeno.

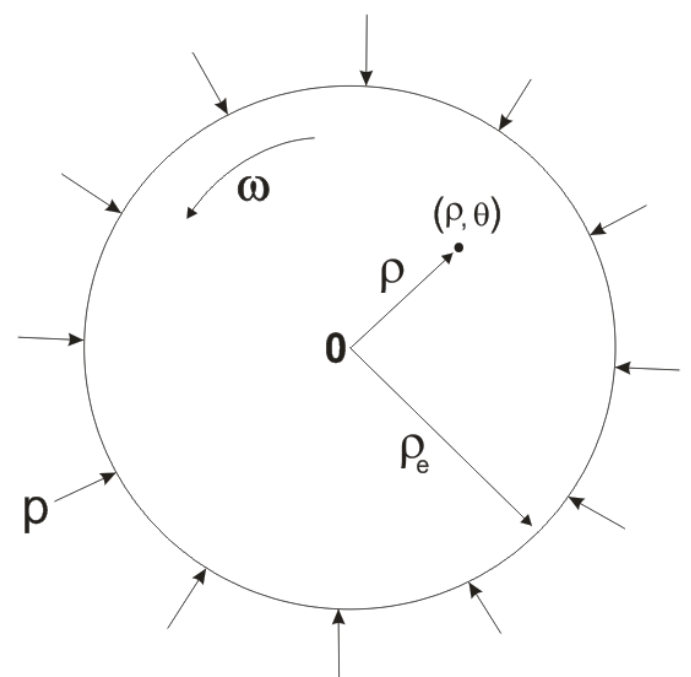

Figura 2.4. Problema do disco girando com velocidade angular constante e sob pressão radial uniformemente distribuída. 
Silva (2007) aplica o método de Galerkin descontínuo para obter soluções aproximadas para o problema do disco girando a uma velocidade angular constante. A partir da análise de convergência dos resultados obtidos o autor conclui que o método de Galerkin descontínuo fornece melhores aproximações para a solução exata do problema do que o método de Galerkin clássico.

Aguiar e Fosdick (2008) estudam o equilíbrio de um tubo circular anisotrópico, homogêneo, com superfície interna fixa, comprimido radialmente por uma força normal uniformemente distribuída $\mathrm{p}$ no seu contorno externo (Fig. 2.5). As soluções obtidas para este problema, considerando-se deformações radialmente simétricas em relação ao centro do tubo, predizem a auto-intersecção da matéria na região próxima à superfície interna do tubo. Os autores analisam o comportamento da solução do problema à medida que variam o raio interno do tubo e observam que o fenômeno da auto-intersecção ainda persiste, contrariando os resultados obtidos por Tarn (2002). Aguiar e Fosdick (2008a) utilizam a teoria de minimização com restrição proposta for Fosdick e Royer-Carfagni (2001) e obtêm a solução exata para o problema, eliminando a auto-intersecção do material.

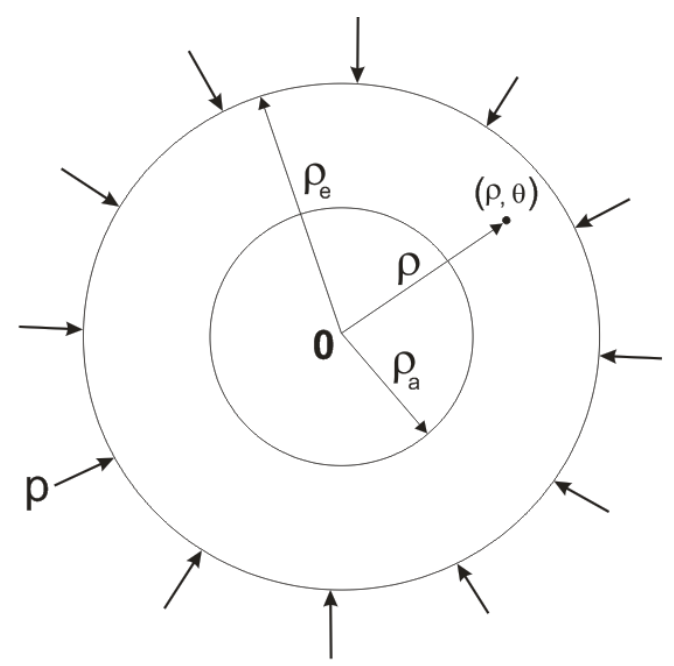

Figura 2.5. Problema do tubo sob pressão radial uniformemente distribuída.

Aguiar, Fosdick e Sánchez (2008) utilizam uma formulação das penalidades interiores juntamente com o método dos elementos finitos para obter soluções aproximadas para o 
problema do tubo estudado por Aguiar e Fosdick (2008a). Os autores modelam ainda o problema do tubo utilizando o software ANSYS 10.0 e observam que o programa fornece soluções que também predizem o fenômeno da auto-intersecção (Fig. 2.6).

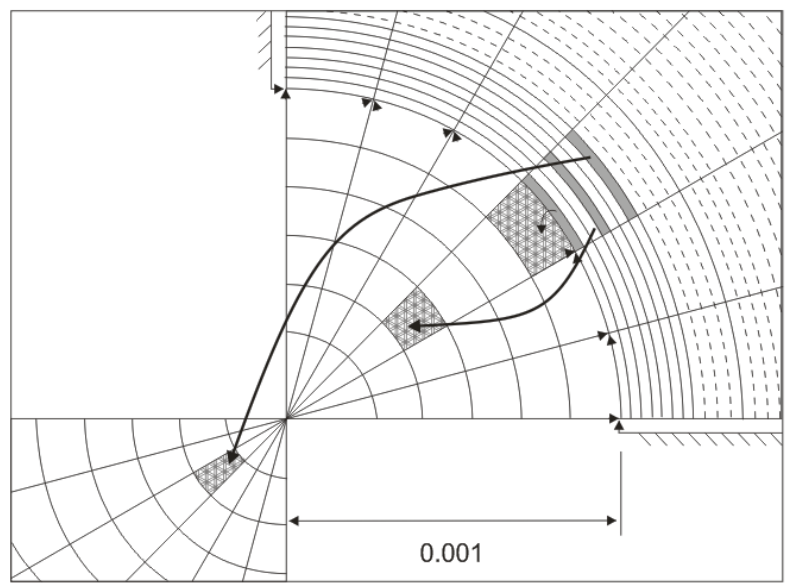

Figura 2.6. Simulação numérica mostrando o fenômeno da auto-intersecção, adaptado de Aguiar, Fosdick e Sánchez (2008).

Fosdick, Freddi e Royer-Carfagni (2008) estudam numericamente o problema do disco de Lekhnitskii (1968) utilizando ambos, a teoria de Fosdick e Royer-Carfagni (2001) e o método das penalidades interiores proposto por Aguiar (2006b), sem considerar que o campo de deslocamento é radialmente simétrico. Eles resolvem um problema plano e encontram resultados numéricos que indicam a existência de uma segunda solução para o problema, ou seja, o campo de deslocamento deixa de ser único, podendo ser radialmente simétrico, ou, rotacionalmente simétrico no contexto da teoria plana com restrição.

\subsection{MÉTODO DOS ELEMENTOS FINITOS DE GALERKIN DESCONTÍNUO}

O estudo dos métodos de Galerkin descontínuo tem motivação nos problemas da mecânica dos fluidos cujas soluções apresentam elevados gradientes, ou, descontinuidades. Diferentemente do método de Galerkin clássico, o método de Galerkin descontínuo evita, ou, reduz o aparecimento de oscilações introduzidas nas soluções numéricas por estas 
características das soluções analíticas dos problemas. O Método dos Elementos Finitos de Galerkin Descontínuo (MEFGD) foi introduzido por Reed e Hill em 1973 para resolver a equação de transporte de nêutrons. Uma variedade de métodos de Galerkin descontínuo foi desde então proposta para a resolução de problemas hiperbólicos e quase-hiperbólicos (REED; HILL, 1973; LESAINT; RAVIART, 1974, etc.).

Para equações diferenciais elípticas, o desenvolvimento dos métodos de Galerkin descontínuo deu-se de forma independente, sendo inicialmente chamado de Método das Penalidades Interiores (ARNOLD, 1976, BAKER, 1977, DOUGLAS; DUPONT, 1976; WHEELER, 1978). As penalidades foram introduzidas ao método de elementos finitos como um meio de impor fracamente as condições de contorno de Dirichlet, em vez de incorporá-las diretamente no espaço de elementos finitos (ARNOLD; BREZZI; COCKBURN; MARINI, 2000).

No método de Galerkin descontínuo a continuidade da solução nas interfaces dos elementos do domínio não é imposta diretamente através do espaço de interpolação, mas de forma fraca, através de fluxos numéricos, na formulação variacional (FORTI, 2005).

Os fluxos numéricos são responsáveis pelas propriedades numéricas do método, tais como estabilidade, ordem de convergência, simetria, etc., (CASTILLO, 2003) e é a partir deles que se definem os diferentes métodos de Galerkin descontínuo introduzidos para a resolução aproximada de equações diferenciais elípticas. Como exemplo, pode-se citar o Método das Penalidades Interiores (MPI), o Método de Elemento Global (MEG), o Método de Bassi e Rebay, o Método de Galerkin Descontínuo Local (MGDL), o Método de Baumann e Oden, introduzidos respectivamente por (WHEELER, 1978, DELVES; HALL, 1979, BASSI; REBAY, 1997, COCKBURN; SHU, 1998, BAUMANN; ODEN, 1999), entre outros.

Os métodos de Galerkin descontínuo são localmente conservativos, estáveis, paralelizáveis, apresentam alta ordem de precisão, adaptam-se facilmente a geometrias 
complexas e malhas irregulares e suportam aproximações com polinômios de graus diferentes em elementos diferentes, tornando-os ideais para o uso em estratégias hp-adaptativas (COCKBURN, 2003).

A literatura disponível sobre os métodos de Galerkin descontínuo é vastíssima. Cockburn, Karniadakis e Shu (2000) apresentam um histórico da evolução destes métodos desde a sua introdução em 1973. Os autores apresentam aspectos teóricos e numéricos dos métodos aplicados na resolução aproximada de equações diferenciais hiperbólicas, parabólicas e elípticas.

Arnold, Brezzi, Cockburn e Marini (2000) e Arnold, Brezzi, Cockburn e Marini (2002) apresentam uma análise unificada das características de vários métodos de Galerkin descontínuo propostos para o tratamento numérico de problemas elípticos.

Relacionam-se abaixo, em um sistema autor-data, alguns trabalhos aplicados ao estudo de equações diferenciais elípticas, ou, de equações que contenham operadores elípticos em sua estrutura. Objetiva-se descrevê-los e evidenciar que embora alguns autores afirmem a superioridade dos métodos de Galerkin descontínuo aplicados ao estudo de problemas de elasticidade, poucos trabalhos desta natureza estão disponíveis na literatura.

Wheeler (1978) propõe um método das penalidades interiores para a resolução aproximada de equações elípticas utilizando espaços de funções polinomiais descontínuas. A formulação apresentada por Wheeler apresenta as seguintes desvantagens: perda da propriedade da conservação ao nível do elemento, as taxas de convergência e a estabilidade do método dependem do parâmetro de penalização e as matrizes resultantes são mal condicionadas.

Delves e Hall (1979) apresentam o Método de Elemento Global (MEG), que consiste em uma formulação híbrida clássica para um problema de Poisson onde o multiplicador de Lagrange é substituído pela média do fluxo nas fronteiras entre elementos. A maior 
desvantagem desta formulação é que a matriz resultante é indefinida, o que torna o método inadequado para resolver problemas de difusão dependentes do tempo. Além disto, sendo a matriz indefinida, os sistemas lineares associados ao problema de difusão em regime permanente precisam de esquemas iterativos especiais (BABUSKA; BAUMANN; ODEN, 1999).

Babuska, Baumann e Oden (1999) apresentam uma análise matemática para uma variação do método de Galerkin descontínuo aplicado na resolução numérica de problemas de difusão. O método proposto é uma modificação do MEG apresentado por Delves e Hall (1979) e não apresenta as deficiências do método de Wheeler (1978). A partir da análise de estabilidade os autores concluem que o método é robusto e fazem estimativa de erro a priori nos espaços finito e infinito-dimensional e obtêm taxa de convergência ótima.

Brezzi, Manzini, Marini e Russo (2000) analisam o método dos elementos finitos descontínuos para resolução de equações elípticas proposto por Bassi e Rebay (1997). Os autores estudam o problema modelo

$$
-\Delta \mathrm{u}=\mathrm{f} \text { em } \Omega, \mathrm{u}=\mathrm{g} \text { sobre } \partial_{1} \Omega, \frac{\partial \mathrm{u}}{\partial \mathrm{n}}=\mathrm{g} \cdot \mathrm{n} \text { sobre } \partial_{2} \Omega, \partial \Omega=\partial_{1} \Omega \cup \partial_{2} \Omega,
$$

para o qual provam estabilidade do método e fazem análise de convergência em várias normas.

Castillo, Cockburn, Perugia e Schötzau (2000) apresentam a primeira análise de erro $a$ priori para o método de Galerkin descontínuo local (MGDL) introduzido por Cockburn e Shu (1998) para a resolução de sistemas convecção-difusão. Os autores analisam o método aplicado ao problema modelo puramente elíptico (2.2) e obtêm taxas de convergência ótimas para os parâmetros estudados e os experimentos numéricos realizados confirmam os resultados teóricos obtidos. 
Duarte, Rochinha e Carmo (2000) utilizam o método dos elementos finitos descontínuos para aproximar a solução de um problema de valor de contorno da elasticidade que possui domínio fissurado (Fig. 2.7). Em geral, as soluções de tais problemas não são suaves na vizinhança da fissura. A partir dos excelentes resultados numéricos obtidos, os autores confirmam que as formulações com elementos finitos descontínuos são muito apropriadas para o estudo de problemas com tais características e que se mostram promissores para o estudo de problemas que requerem esquemas adaptativos.
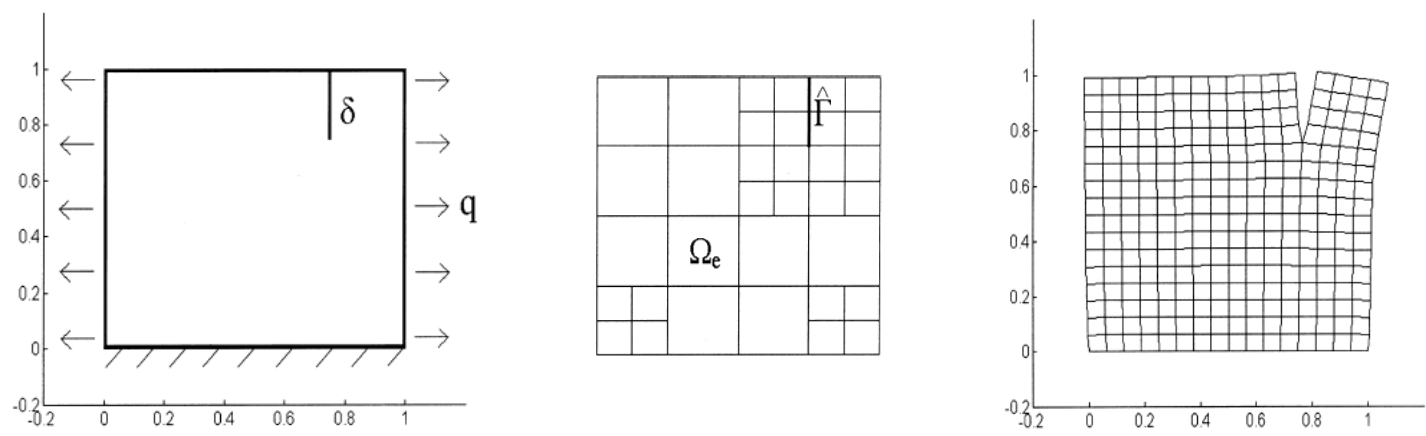

Figura 2.7. Problema estudado por Duarte, Rochinha e Carmo (2000).

Rivière, Wheeler e Girault (2001) analisam três aproximações do método de Galerkin descontínuo para problemas elípticos bi e tridimensionais. As formas bilineares destas aproximações são não-simétricas. Os autores obtêm estimativas de erros ótimas para as três formulações nas normas $\mathrm{H}_{1}$ e $\mathrm{L}_{2}$.

Cockburn, Kanschat, Perugia e Schötzau (2001) estudam o método de Galerkin descontínuo local aplicado ao problema modelo puramente elíptico (2.2) em domínio Cartesiano. Os autores identificam um fluxo especial para o qual a taxa de convergência do gradiente na norma $\mathrm{L}_{2}$ é $\mathrm{k}+1 / 2$ quando polinômios de grau $\mathrm{k}$ são utilizados.

Castillo, Cockburn, Perugia e Schötzau (2002) revisam o método de Galerkin descontínuo local aplicado ao problema modelo puramente elíptico (2.2). Os autores apresentam a formulação do método e um estudo de erros. Como principais vantagens do 
MGDL aplicado ao estudo de problemas puramente elípticos podem-se citar a facilidade com que o método se adapta a geometrias gerais e a flexibilidade com que suporta aproximações com polinômios de graus diferentes em elementos diferentes. Estas propriedades tornam o MGDL ideal para o desenvolvimento de estratégias numéricas hp-adaptativas e para o acoplamento com outros métodos. Neste sentido, os autores mostram como acoplar o MGDL com o método de Galerkin clássico explorando as vantagens do MGDL com um custo computacional reduzido. O esquema apresentado pelos autores fornece boas taxas de convergência. Exemplos numéricos confirmam os resultados teóricos obtidos pelos autores.

Cockburn, Kanschat e Schötzau (2002) propõem e analisam um novo método de Galerkin descontínuo local para as equações de Navier-Stokes incompressíveis estacionárias. O método proposto pelos autores é estável, localmente conservativo, apresenta alta ordem de precisão e satisfaz exatamente a restrição da incompressibilidade. Além disso, prova-se estimativa de erro ótima e o procedimento iterativo resultante converge para a solução exata do problema.

Castillo (2003) apresenta um estudo teórico e numérico de uma classe de métodos de Galerkin descontínuo aplicados ao problema de valor de contorno da forma

$$
-\mathrm{u}^{\prime \prime}=\mathrm{f} \text { em } \Omega=(0,1), \mathrm{u}(0)=\mathrm{u}_{0}, \mathrm{u}(1)=\mathrm{u}_{1},
$$

onde $\mathrm{u}_{0}$ e $\mathrm{u}_{1}$ são valores conhecidos. Utilizando experimentos numéricos o autor mostra que a aproximação do gradiente $\mathrm{q}=\mathrm{u}^{\prime}$ introduzida ao problema superconverge para os zeros do polinômio de Legendre, confirmando os resultados teóricos obtidos.

Krindges (2004) estuda quatro variações do método de Galerkin descontínuo aplicadas a equações lineares elípticas de segunda ordem em domínio bidimensional. As variações estudadas pelo autor são o Método de Elementos Global (MEG), Método de Galerkin 
Simétrico com Penalização Interior (MGSPI), hp-Método de Galerkin Descontínuo (GD) e Método de Galerkin Não-Simétrico com Penalização Interior (MGPIN). O autor obtém estimativas de erro a priori na norma de energia e na norma $\mathrm{L}_{2}$ para três destas formulações, (MGSPI), (GD) e (MGPIN), e, a partir de experimentos numéricos realizados, o autor obtém taxas de convergência ótimas nas normas consideradas.

Mozolevski e Bösing (2005) apresentam uma formulação do método de Galerkin descontínuo para as equações de Navier-Stokes incompressíveis, bidimensionais em regime permanente. Para resolver esta equação os autores combinam duas formulações, uma para a parte elíptica e outra para a parte hiperbólica do problema. Esta combinação resulta em um método de elementos finitos de Galerkin descontínuo com penalidade interior. Resultados numéricos obtidos confirmam a eficiência do método para uma ampla faixa de números de Reynolds.

Forti (2005) aplica o método de Galerkin descontínuo no estudo de problemas de convecção-difusão e obtém bons resultados. Em particular, o autor aplica o método para resolução de problemas de camada limite e observa a redução da propagação de oscilações para além da região de elevados gradientes. O autor observa ainda que ao se alinhar as interfaces entre elementos finitos com a descontinuidade da solução as oscilações desaparecem. Considerando a desvantagem do grande número de graus de liberdade requeridos no método de Galerkin descontínuo, o autor acopla o método de Galerkin clássico com o método de Galerkin descontínuo objetivando extrair as melhores características de cada um, ou seja, na região de elevados gradientes o autor utiliza o método de Galerkin descontínuo buscando a estabilidade da solução e na região de solução suave utiliza o método de Galerkin clássico para reduzir o número de graus de liberdade. $\mathrm{O}$ acoplamento adotado pelo autor mostrou-se satisfatório.

Bösing (2006) estuda o método de Galerkin Descontínuo aplicado a equações elípticas 
de alta ordem. O autor apresenta as formulações com penalização interior, simétrica, nãosimétrica e duas semi-simétricas e desenvolve análise de estabilidade e estimativa de erro $a$ priori na norma de energia para todas elas. $\mathrm{O}$ autor desenvolve duas formulações de Galerkin descontínuo para uma equação de quarta ordem não-linear obtida das equações de NavierStokes bidimensionais, incompressíveis, em regime estacionário. O autor emprega o método de Newton-Raphson e observa que ambas as formulações desenvolvidas são eficientes na resolução numérica de alguns problemas clássicos da mecânica dos fluidos computacional.

Castillo (2006) apresenta uma revisão do método de Galerkin descontínuo local (MGDL) aplicado ao problema elíptico modelo

$$
-\nabla \cdot \sigma \nabla \mathrm{u}=\mathrm{f} \text { em } \Omega, \mathrm{u}=\mathrm{g} \text { sobre } \partial \Omega
$$

e inicia o estudo de existência de pontos convergentes. Os resultados numéricos obtidos pelo autor mostram que a posição destes pontos depende de um parâmetro $\beta$. A análise teórica destes resultados permanece um problema em aberto.

Schneider, Xu e Zhou (2006) estudam o comportamento global e local de três tipos de métodos de Galerkin descontínuo para equações elípticas de segunda ordem. Estimativas de erro a posteriori são apresentadas. Os autores aplicam os resultados teóricos no desenvolvimento de um esquema numérico adaptativo-paralelo.

Mozolevski, Bösing e Schuh (2007) introduzem uma nova formulação do método de Galerkin descontínuo para problemas elípticos de segunda ordem que penaliza simultaneamente os saltos da solução e os saltos dos fluxos da solução numérica. A análise de erro a priori do método introduzido mostra taxa de convergência ótima em h e quase-ótima em p. Experimentos numéricos confirmam as taxas de convergência obtidas.

Gudi, Nataraj e Pani (2008) analisam uma família de métodos de Galerkin descontínuo 
hp-adaptativos para problemas de valor de contorno elípticos altamente não-lineares. A partir da análise de erro, os autores mostram que tanto para o problema linear quanto para o problema não-linear a estimativa de erro é ótima em p e sub-ótima em h. Experimentos numéricos confirmam os resultados teóricos obtidos. 


\subsection{CONSIDERAÇÕES INICIAIS}

Por elasticidade entende-se a propriedade que um meio material apresenta de restaurar a sua condição inicial indeformada quando cessada a ação de um sistema de forças externas ao qual foi submetido. Corpos que apresentam este tipo de comportamentamento são chamados perfeitamente elásticos. Muitos materiais utilizados em engenharia apresentam até certo grau a propriedade da elasticidade (TIMOSHENKO; GOODIER, 1980).

Seja $\Omega \subset \mathrm{R}^{3}$ a configuração de referência natural indeformada de um corpo e $\partial \Omega$ o seu contorno, de tal forma que $\Omega \cap \partial \Omega=\varnothing$. Os pontos $\mathrm{x} \in \Omega$ são mapeados em pontos $\hat{\mathrm{x}}=\mathrm{f}(\mathrm{x}) \equiv \mathrm{x}+\mathrm{u}(\mathrm{x}) \in \mathrm{R}^{3}$, onde $\mathrm{u}(\mathrm{x})$ é o deslocamento de $\mathrm{x}$. O contorno de $\Omega$, $\partial \Omega$, é composto de duas partes complementares $\partial_{1} \Omega$ e $\partial_{2} \Omega$, de tal forma que $\partial_{1} \Omega \cup \partial_{2} \Omega=\partial \Omega$ e $\partial_{1} \Omega \cap \partial_{2} \Omega=\varnothing$. As condições de contorno em deslocamento $\overline{\mathrm{u}}(\mathrm{x})$, ou condições de contorno de Dirichlet, são prescritas em pontos $x \in \partial_{1} \Omega$ e as condições de contorno em forças $\overline{\mathfrak{t}}(\mathrm{x})$, ou, condições de contorno de Newmann são prescritas em pontos $\mathrm{x} \in \partial_{2} \Omega$. A força de corpo $\mathrm{b}(\mathrm{x})$ por unidade de volume de $\Omega$ atua em pontos $\mathrm{x} \in \Omega$.

Aplica-se a Teoria da Elasticidade Linear Clássica no estudo de corpos elásticos 
submetidos à ação de sistemas de forças externas conhecidas com o objetivo de determinar os campos de deslocamento, tensão e deformação, que descrevem completamente o comportamento destes corpos. A forma, as dimensões e as constantes elásticas do material do qual o corpo é constituído também são conhecidos.

Considera-se ainda que o material é homogêneo, ou seja, todos os seus pontos possuem as mesmas propriedades físicas específicas e que a matéria é distribuída continuamente no seu volume, ou seja, não há vazios.

Para determinar as três componentes de deslocamento, as seis componentes de tensão e as seis componentes de deformação em um ponto de um corpo tridimensional, têm-se três equações de equilíbrio, seis relações deformação-deslocamento e seis relações tensãodeformação. Estas equações são apresentadas neste capítulo, bem como alguns conceitos necessários para a obtenção das mesmas (ODEN, 1967; VALLIAPPAN, 1981; VILLAÇA; GARCIA, 2000; SOKOLNIKOFF, 1956; etc.). Devido à natureza do problema aqui estudado, as equações básicas da elasticidade são apresentadas no sistema de coordenadas esféricas.

Por fim, apresenta-se a aplicação da teoria no estudo do problema da esfera anisotrópica sob compressão radial uniformemente distribuída considerando-se deformações radialmente simétricas e material esfericamente uniforme (AGUIAR, 2006b; TING, 1999).

\subsection{PONTO NO ESPAÇO EM COORDENADAS ESFÉRICAS}

Seja $\mathrm{P}=\mathrm{P}(\mathrm{x}, \mathrm{y}, \mathrm{z})$ um ponto no espaço euclidiano tridimensional (Fig. 3.1). A posição do ponto em relação à origem pode ser descrita em termos das coordenadas esféricas, $\mathrm{P}=\mathrm{P}(\rho, \phi, \theta)$, onde

$$
\rho=\sqrt{x^{2}+y^{2}+z^{2}}, \phi=\operatorname{arctg} \frac{y}{x}, \theta=\operatorname{arctg}\left(\frac{\sqrt{x^{2}+y^{2}}}{z}\right),
$$


sendo $\rho>0,0<\phi \leq 360^{\circ}$ e $0<\theta<180^{\circ}$.

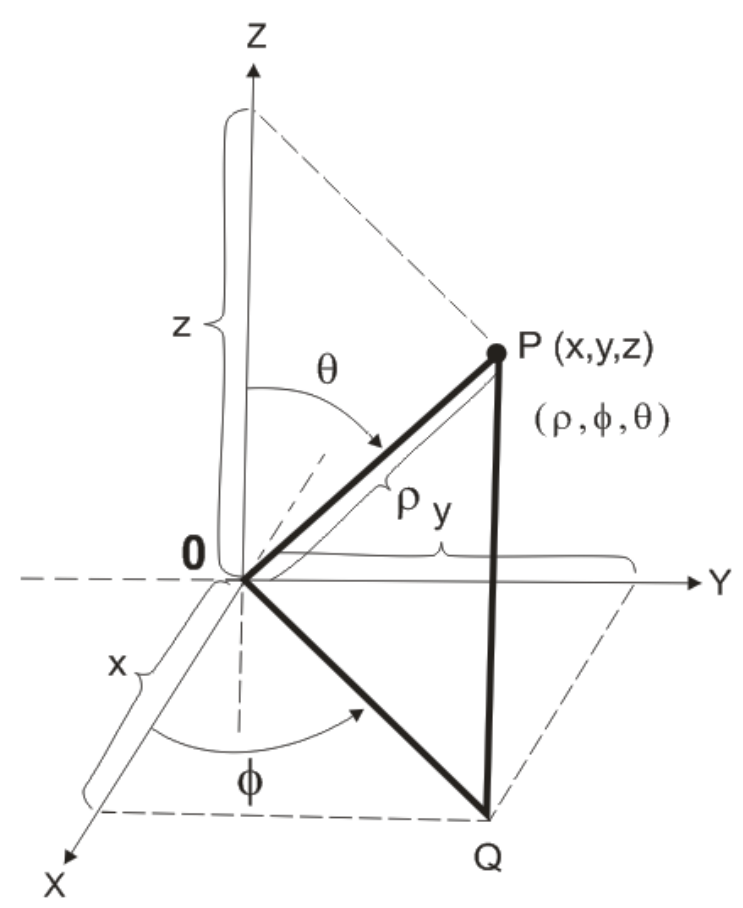

Figura 3.1. Ponto no espaço em coordenadas esféricas.

Invertendo as relações (3.1), obtém-se

$$
x=\rho \operatorname{sen} \theta \cos \phi, y=\rho \operatorname{sen} \theta \operatorname{sen} \phi, z=\rho \cos \theta .
$$

\subsection{CONCEITOS FUNDAMENTAIS}

Ao se estudar um determinado sistema tem-se por objetivo descrever o seu comportamento. Por comportamento entende-se a forma como este sistema responde a um determinado estímulo. Aqui, o estímulo será a ação das forças externas atuantes e o comportamento que se deseja determinar é descrito pelos campos de tensão, deslocamento e deformação resultantes da ação destas forças externas. 
Descrevem-se então cada uma destas grandezas, forças, deslocamentos, tensão e deformação, independentemente, para posteriormente, descrever a forma como elas interagem entre si.

\subsubsection{FORÇAS}

As forças são responsáveis por alterar o estado de repouso, ou, movimento de um corpo, gerar o estado de tensão interna e mudar a forma do corpo devido aos deslocamentos das suas partículas.

Basicamente, para o tipo de sistema aqui estudado, estas forças podem ser internas, ou, externas. As forças externas podem ser de superfície, ou, de volume.

Seja um corpo em equilíbrio sob a ação de um sistema de forças externas aplicadas (Fig. 3.2a). Seccionando-se este corpo por um plano $\mathrm{S}$ e analisando-se cada uma das partes separadamente (Fig. 3.2b) observa-se que ambas estão em equilíbrio devido ao surgimento de forças internas interativas entre as partículas do corpo e da ação de uma parte sobre a outra. Considera-se que a força interna $\mathrm{F}$ é a resultante das forças continuamente distribuídas que atuam sobre S. Estas resultantes internas $\mathrm{F}$ possuem a mesma intensidade, a mesma direção e sentidos opostos (Fig. 3.2b).
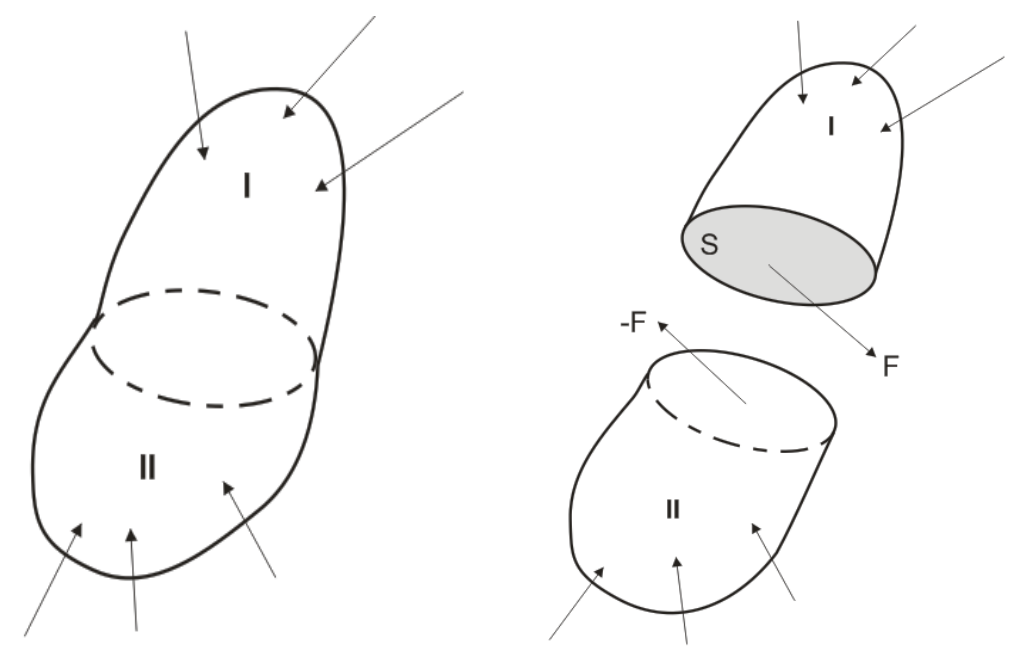

Figura 3.2. Corpo seccionado sob a ação de forças internas e externas. 


\subsubsection{CONCEITO DE TENSÃO}

A ação das forças externas em um corpo gera interação interna entre suas partículas, que são descritas por tensões.

Para introduzir o conceito de tensão considera-se um plano $\mathrm{S}$ que intersecciona um corpo em duas partes, I e II (Fig. 3.3). Seja $\Delta S$ um elemento de área contido no plano $\mathrm{S}$ e que contém o ponto $\mathrm{O}$ (Fig. 3.3). Seja $\Delta \mathrm{F}$ a resultante das forças internas que atuam em $\Delta \mathrm{S}$. A tensão média resultante das forças internas que atuam em $\Delta \mathrm{S}$ é dada por:

$$
\sigma_{\mathrm{m}}=\frac{\Delta \mathrm{F}}{\Delta \mathrm{S}}
$$

Fazendo-se a área $\Delta \mathrm{S}$ diminuir indefinidamente, no limite, chega-se à tensão que atua no ponto $\mathrm{O}$ dada por:

$$
\sigma=\frac{\mathrm{dF}}{\mathrm{dS}}=\lim _{\Delta \mathrm{S} \rightarrow 0} \frac{\Delta \mathrm{F}}{\Delta \mathrm{S}}
$$

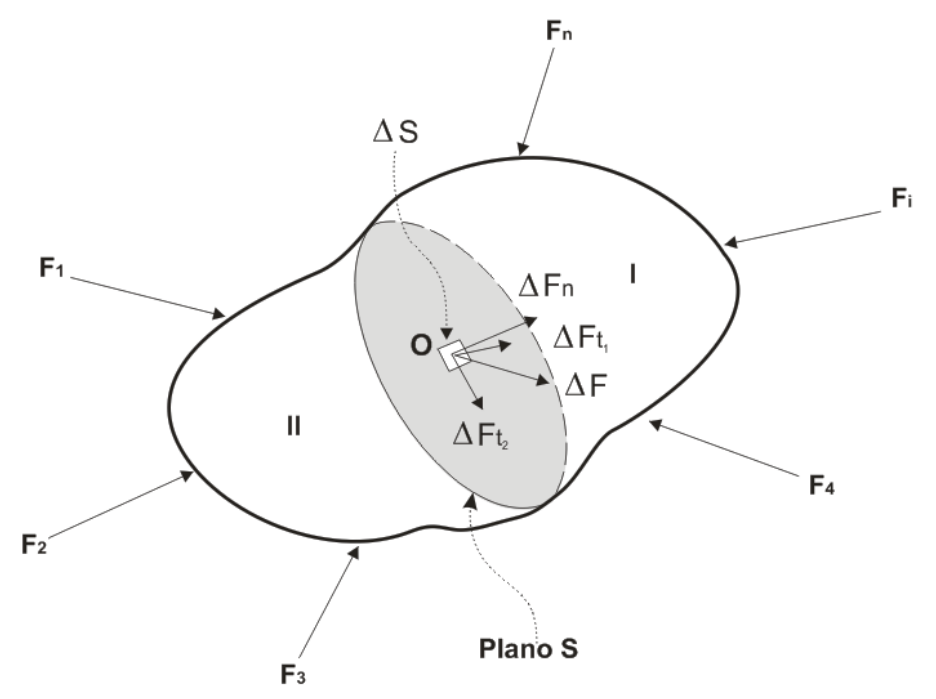

Figura 3.3. Corpo sob a ação de forças externas. 
Decompondo-se o vetor $\Delta \mathrm{F}$ nas direções normal e tangenciais ao plano $\mathrm{S}$ (Fig. 3.3) e utilizando-se a definição (3.4) chega-se às componentes de tensão normal, $\sigma_{n}$, e tangenciais, $\tau_{1}$ e $\tau_{2}$, dadas, respectivamente, por:

$$
\sigma_{\mathrm{n}}=\lim _{\Delta S \rightarrow 0} \frac{\Delta \mathrm{F}_{\mathrm{n}}}{\Delta \mathrm{S}}, \tau_{1}=\lim _{\Delta \mathrm{S} \rightarrow 0} \frac{\Delta \mathrm{F}_{\mathrm{t}_{1}}}{\Delta \mathrm{S}}, \tau_{2}=\lim _{\Delta S \rightarrow 0} \frac{\Delta \mathrm{F}_{\mathrm{t}_{2}}}{\Delta \mathrm{S}}
$$

\subsubsection{DESLOCAMENTO}

A posição ocupada simultaneamente por todas as partículas de um sistema, em um dado tempo $\mathrm{t}$, define a configuração do sistema.

Seja A um ponto de um corpo na sua configuração inicial indeformada (Fig. 3.4). com coordenadas $\left(\mathrm{x}_{0}, \mathrm{y}_{0}, \mathrm{z}_{0}\right)$.

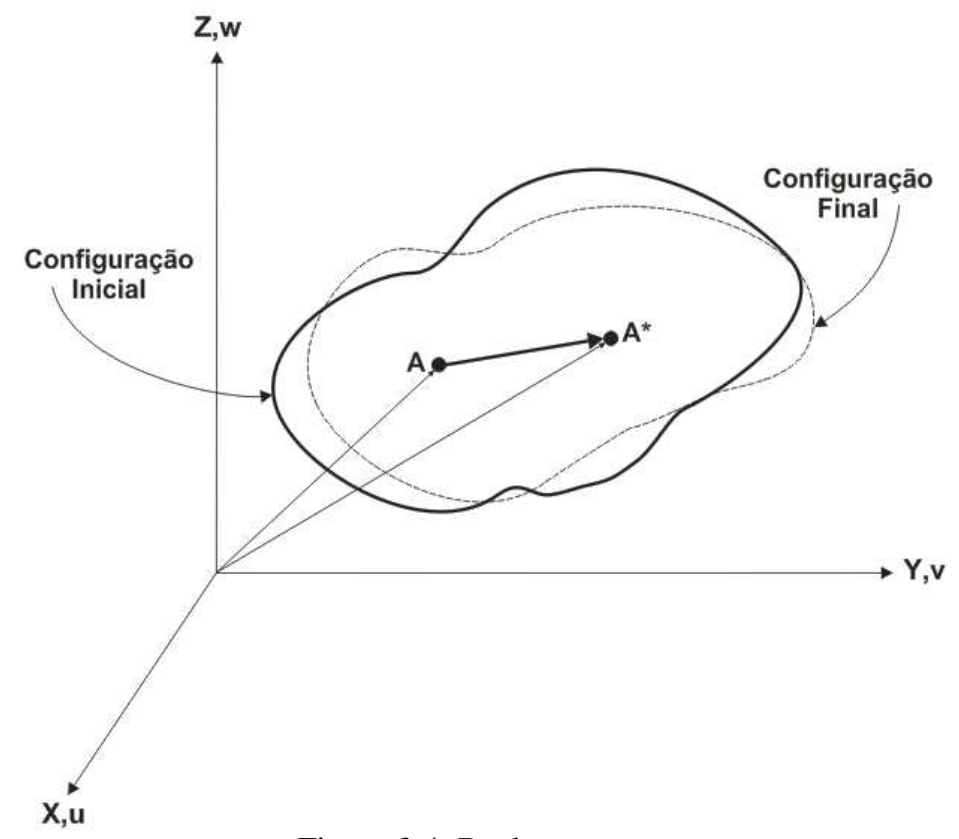

Figura 3.4. Deslocamento.

Sob a ação de um sistema de forças externas aplicadas, o corpo muda de configuração. 
Em particular, o ponto A desloca-se para um ponto $\mathrm{A}^{*}$, com coordenadas $\left(\mathrm{x}_{0}+\mathrm{u}, \mathrm{y}_{0}+\mathrm{v}, \mathrm{z}_{0}+\mathrm{w}\right)$

O deslocamento $(\mathrm{u}, \mathrm{v}, \mathrm{w})$ de um ponto pode, então, ser definido como uma mudança de posição deste ponto em relação a um sistema de referência fixo no espaço e é uma grandeza vetorial caracterizada por uma direção, um sentido e uma intensidade.

Em um sistema de coordenadas esféricas o campo de deslocamento, $\left(\mathrm{u}_{\rho}, \mathrm{u}_{\phi}, \mathrm{u}_{\theta}\right)$, é descrito pelas funções

$$
\mathrm{u}_{\rho}=\mathrm{u}_{\rho}(\rho, \phi, \theta), \mathrm{u}_{\theta}=\mathrm{u}_{\theta}(\rho, \phi, \theta), \mathrm{u}_{\phi}=\mathrm{u}_{\phi}(\rho, \phi, \theta)
$$

\subsubsection{DEFORMAÇÃO}

A mudança na forma de um corpo entre uma configuração inicial e uma configuração final é conhecida como deformação. À medida que o corpo é transformado da configuração inicial para a configuração final, a matéria na vizinhança de cada ponto do corpo é transladada e rotacionada e consequentemente deformada.

As componentes da deformação em um ponto são descritas pelos alongamentos $\varepsilon$ e distorções $\gamma_{\mathrm{ts}}$ sofridos por este ponto em relação a um sistema de referência.

O alongamento ou deformação linear específica $\varepsilon$ é dado pelo quociente da deformação linear de um segmento elementar pelo comprimento inicial desse segmento (Fig. 3.5) e pode ser expresso por

$$
\varepsilon=\frac{\overline{A^{*} B^{*}}-\overline{A B}}{\overline{A B}}=\frac{d_{s}^{*}-d_{s}}{d_{s}} .
$$




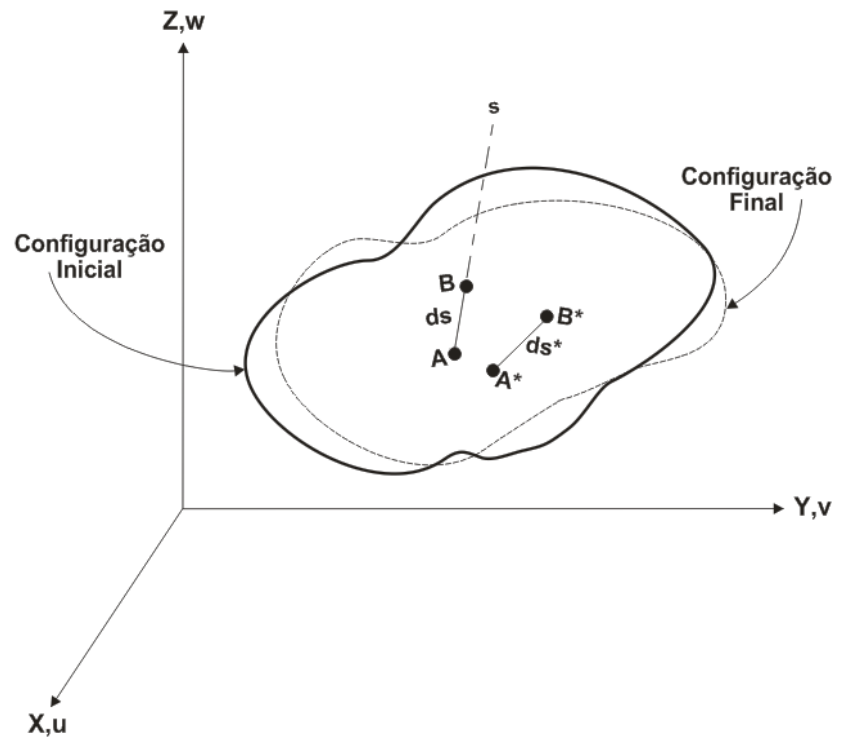

Figura 3.5. Deformação linear.

A distorção, ou, deformação angular $\gamma_{\mathrm{ts}}$ representa a variação do ângulo formado por duas direções inicialmente perpendiculares, definidas em um ponto de um corpo, após a deformação deste corpo (Fig. 3.6). A deformação angular é expressa por:

$$
\gamma_{\mathrm{ts}}=\frac{\pi}{2}-\mathrm{d} \hat{\mathrm{t}}
$$

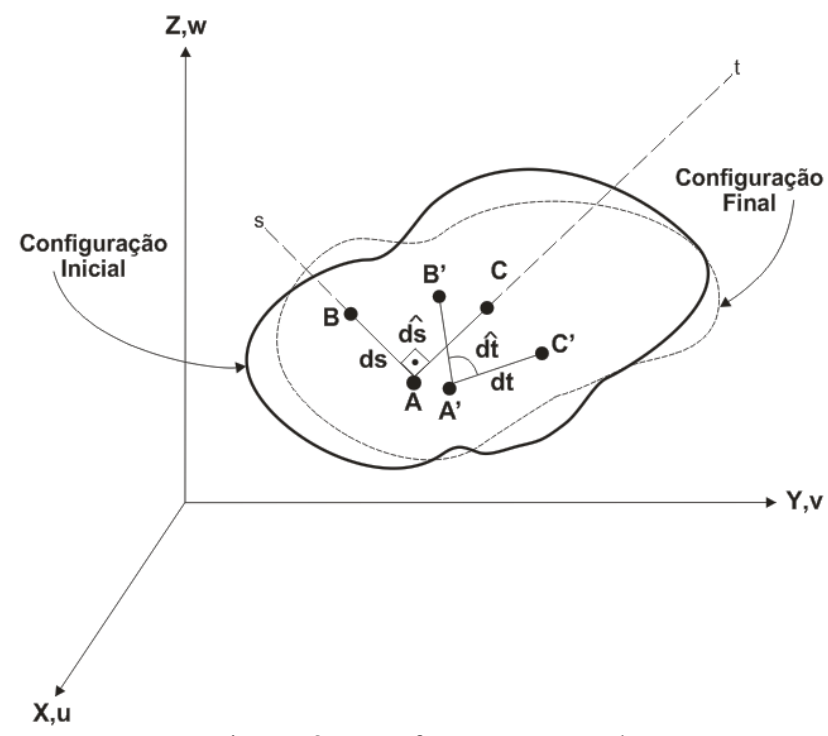

Figura 3.6. Deformação angular.

A partir das definições (3.7) e (3.8) determinam-se, respectivamente, as expressões das três componentes lineares e das três componentes angulares de deformação associadas a um 
ponto de um corpo. Em um sistema de coordenadas esféricas, estas componentes $\left(\varepsilon_{\rho \rho}, \varepsilon_{\phi \phi}, \varepsilon_{\theta \theta}, \varepsilon_{\theta \phi}, \varepsilon_{\phi \rho}, \varepsilon_{\rho \theta}\right)$ ficam completamente descritas pelas funções

$$
\begin{aligned}
& \varepsilon_{\rho \rho}=\varepsilon_{\rho \rho}(\rho, \phi, \theta), \varepsilon_{\phi \phi}=\varepsilon_{\phi \phi}(\rho, \phi, \theta), \varepsilon_{\theta \theta}=\varepsilon_{\theta \theta}(\rho, \phi, \theta), \\
& \varepsilon_{\rho \theta}=\varepsilon_{\rho \theta}(\rho, \phi, \theta), \varepsilon_{\rho \phi}=\varepsilon_{\rho \phi}(\rho, \phi, \theta), \varepsilon_{\theta \phi}=\varepsilon_{\theta \phi}(\rho, \phi, \theta) .
\end{aligned}
$$

\subsection{EQUAÇÕES BÁSICAS DA TEORIA DA ELATICIDADE LINEAR CLÁSSICA}

Uma vez conhecidos os conceitos das grandezas, tensão, deslocamento e deformação, descrevem-se como estas grandezas interagem entre si. Estas iterações são descritas pelas equações de equilíbrio, pelas equações deslocamento-deformação e pelas equações constitutivas apresentadas nesta seção.

\subsubsection{EQUAÇÕES DE EQUILÍBRIO EM COORDENADAS ESFÉRICAS}

Em um sistema de coordenadas esféricas, $(\rho, \phi, \theta)$, as equações de equilíbrio são dadas por

$$
\begin{aligned}
& \frac{\partial \sigma_{\rho \rho}}{\partial \rho}+\frac{1}{\rho} \frac{\partial \sigma_{\theta \rho}}{\partial \theta}+\frac{1}{\rho \operatorname{sen} \theta} \frac{\partial \sigma_{\phi \rho}}{\partial \phi}+\frac{1}{\rho}\left(2 \sigma_{\rho \rho}-\sigma_{\theta \theta}-\sigma_{\phi \phi}+\sigma_{\theta \rho} \cot \theta\right)+b_{\rho}=0, \\
& \frac{\partial \sigma_{\rho \phi}}{\partial \rho}+\frac{1}{\rho} \frac{\partial \sigma_{\theta \phi}}{\partial \theta}+\frac{1}{\rho \operatorname{sen} \theta} \frac{\partial \sigma_{\phi \phi}}{\partial \phi}+\frac{1}{\rho}\left(2 \sigma_{\theta \phi} \cot \theta+3 \sigma_{\rho \phi}\right)+b_{\phi}=0, \\
& \frac{\partial \sigma_{\rho \theta}}{\partial \rho}+\frac{1}{\rho} \frac{\partial \sigma_{\theta \theta}}{\partial \theta}+\frac{1}{\rho \operatorname{sen} \theta} \frac{\partial \sigma_{\phi \theta}}{\partial \phi}+\frac{1}{\rho}\left[\left(\sigma_{\theta \theta}-\sigma_{\phi \phi}\right) \cot \theta+3 \sigma_{\rho \theta}\right]+b_{\theta}=0,
\end{aligned}
$$

onde $b \equiv\left(b_{\rho}, b_{\phi}, b_{\theta}\right)$ é a força de corpo por unidade de volume. 


\subsubsection{RELAÇÕES DEFORMAÇÃO-DESLOCAMENTO EM COORDENADAS ESFÉRICAS}

As relações entre as três componentes de deslocamento $\left(\mathrm{u}_{\rho}, \mathrm{u}_{\phi}, \mathrm{u}_{\theta}\right)$ e as seis componentes de deformação $\left(\varepsilon_{\rho \rho}, \varepsilon_{\phi \phi}, \varepsilon_{\theta \theta}, \varepsilon_{\theta \phi}, \varepsilon_{\phi \rho}, \varepsilon_{\rho \theta}\right)$ são dadas por

$$
\begin{aligned}
& \varepsilon_{\rho \rho}=\frac{\partial u_{\rho}}{\partial \rho}, \varepsilon_{\phi \phi}=\frac{1}{\rho \operatorname{sen} \theta} \frac{\partial u_{\phi}}{\partial \phi}+u_{\theta} \frac{\cot \theta}{\rho}+\frac{u_{\rho}}{\rho}, \varepsilon_{\theta \theta}=\frac{1}{\rho} \frac{\partial u_{\theta}}{\partial \theta}+\frac{u_{\rho}}{\rho}, \\
& 2 \varepsilon_{\theta \phi}=\frac{1}{\rho \operatorname{sen} \theta} \frac{\partial u_{\theta}}{\partial \phi}+\frac{1}{\rho} \frac{\partial u_{\phi}}{\partial \theta}-u_{\phi} \frac{\cot \theta}{\rho} \\
& 2 \varepsilon_{\rho \theta}=\frac{1}{\rho} \frac{\partial u_{\rho}}{\partial \theta}+\frac{\partial u_{\theta}}{\partial \rho}-\frac{u_{\theta}}{\rho}, 2 \varepsilon_{\phi \rho}=\frac{1}{\rho \operatorname{sen} \theta} \frac{\partial u_{\rho}}{\partial \phi}-\frac{u_{\phi}}{\rho}+\frac{\partial u_{\phi}}{\partial \rho} .
\end{aligned}
$$

\subsubsection{EQUAÇÕES CONSTITUTIVAS}

Considerando-se que as equações de equilíbrio (3.10) e as relações deformaçãodeslocamento (3.11) são válidas para qualquer material e que dois corpos, de mesmo tamanho e forma, compostos por materiais diferentes, quando submetidos ao mesmo estímulo, geralmente não apresentam a mesma distribuição de forças resultantes, faz-se necessário introduzir informações a estas relações que permitam distinguir as diferentes respostas para diferentes materiais dos quais os corpos possam ser constituídos e assim, caracterizar completamente o seu comportamento.

Estas informações relacionadas à resposta do material são introduzidas ao problema por meio das equações constitutivas. Estas equações estabelecem relação entre o regime das tensões internas e das deformações, cuja intensidade depende das propriedades mecânicas do material. 
Quando o regime das tensões é proporcional ao regime das deformações, o comportamento do corpo é dito elástico-linear e pode ser descrito pela Lei de Hooke Generalizada, dada por

$$
\left\{\begin{array}{c}
\sigma_{\rho \rho} \\
\sigma_{\phi \phi} \\
\sigma_{\theta \theta} \\
\sigma_{\rho \theta} \\
\sigma_{\rho \phi} \\
\sigma_{\theta \phi}
\end{array}\right\}=\left[\begin{array}{llllll}
\mathrm{C}_{11} & \mathrm{C}_{12} & \mathrm{C}_{13} & \mathrm{C}_{14} & \mathrm{C}_{15} & \mathrm{C}_{16} \\
\mathrm{C}_{21} & \mathrm{C}_{22} & \mathrm{C}_{23} & \mathrm{C}_{24} & \mathrm{C}_{25} & \mathrm{C}_{26} \\
\mathrm{C}_{31} & \mathrm{C}_{32} & \mathrm{C}_{33} & \mathrm{C}_{34} & \mathrm{C}_{35} & \mathrm{C}_{36} \\
\mathrm{C}_{41} & \mathrm{C}_{42} & \mathrm{C}_{43} & \mathrm{C}_{44} & \mathrm{C}_{45} & \mathrm{C}_{46} \\
\mathrm{C}_{51} & \mathrm{C}_{52} & \mathrm{C}_{53} & \mathrm{C}_{54} & \mathrm{C}_{55} & \mathrm{C}_{56} \\
\mathrm{C}_{61} & \mathrm{C}_{62} & \mathrm{C}_{63} & \mathrm{C}_{64} & \mathrm{C}_{65} & \mathrm{C}_{66}
\end{array}\right]\left\{\begin{array}{c}
\varepsilon_{\rho \rho} \\
\varepsilon_{\phi \phi} \\
\varepsilon_{\theta \theta} \\
\varepsilon_{\rho \theta} \\
\varepsilon_{\rho \phi} \\
\varepsilon_{\theta \phi}
\end{array}\right\},
$$

onde $C_{\alpha \beta}, \alpha, \beta=1, \ldots, 6$ são as constantes elásticas do material.

Observa-se de (3.12) que são necessárias 36 constantes elásticas para especificar completamente as propriedades mecânicas de um material elástico-linear, mas que devido à simetria, $\mathrm{C}_{\alpha \beta}=\mathrm{C}_{\beta \alpha}$, somente 21 constantes elásticas são independentes entre si. Um material com tal característica é chamado de anisotrópico, ou seja, suas propriedades elásticas variam em todas as direções. Quando as propriedades apresentam o mesmo valor, independente da direção, o material é denominado isotrópico e as constantes elásticas reduzem-se a 2.

\subsection{A TEORIA DA ELASTICIDADE LINEAR CLÁSSICA APLICADA AO PROBLEMA DA ESFERA}

Seja uma esfera homogênea de raio $\rho_{\mathrm{e}}$ radialmente comprimida ao longo do seu contorno externo por uma força normal uniformemente distribuída por unidade de área $\mathrm{p}$ (Fig. 3.7). Em um sistema de coordenadas esféricas, $(\rho, \phi, \theta)$, as equações de equilíbrio, as relações deformação-deslocamento e as relações tensão-deformação são dadas 
respectivamente, por (3.10), (3.11) e (3.12).

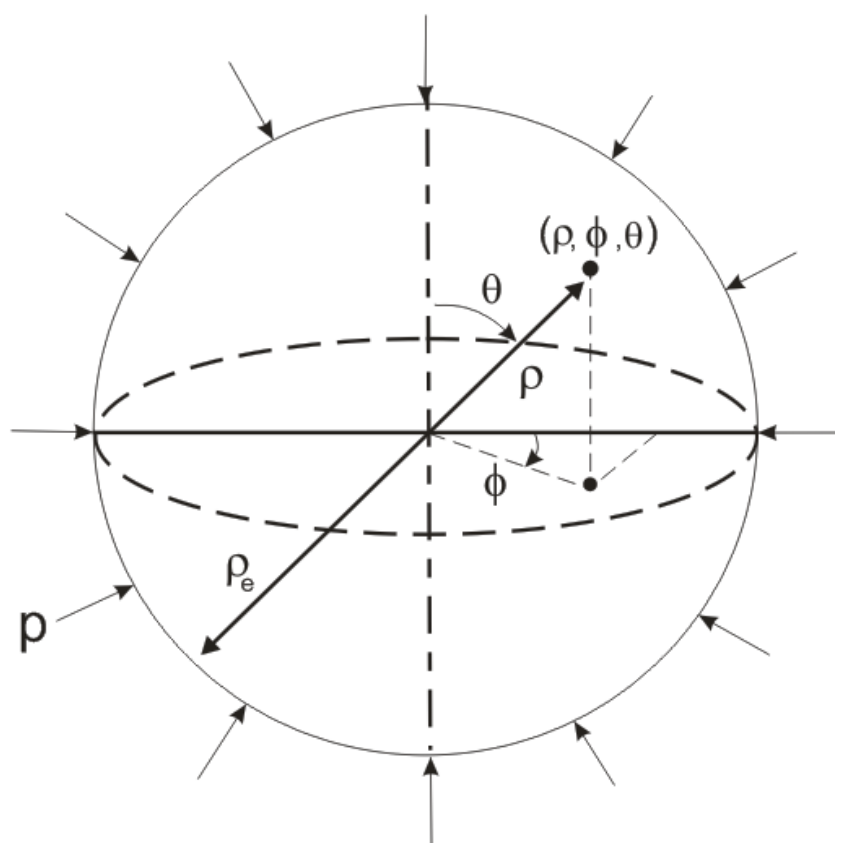

Figura 3.7. Problema da esfera sob pressão radial.

Considera-se que a esfera é constituída pelo material esfericamente uniforme investigado por Ting (1999). Para este material tem-se que

$$
\mathrm{C}_{13}=\mathrm{C}_{12}, \mathrm{C}_{22}=\mathrm{C}_{33} \text { e } \mathrm{C}_{\alpha \beta}=0 \text { para } 1 \leq \alpha \leq 3 \text { e } 4 \leq \beta \leq 6 \text {. }
$$

Desta forma, as relações tensão-deformação dadas por (3.12) passam a ser expressas por

$$
\left\{\begin{array}{c}
\sigma_{\rho \rho} \\
\sigma_{\phi \phi} \\
\sigma_{\theta \theta} \\
\sigma_{\rho \theta} \\
\sigma_{\rho \phi} \\
\sigma_{\theta \phi}
\end{array}\right\}=\left[\begin{array}{cccccc}
C_{11} & C_{12} & C_{12} & 0 & 0 & 0 \\
C_{12} & C_{22} & C_{23} & 0 & 0 & 0 \\
C_{12} & C_{32} & C_{22} & 0 & 0 & 0 \\
0 & 0 & 0 & C_{44} & C_{45} & C_{46} \\
0 & 0 & 0 & C_{54} & C_{55} & C_{56} \\
0 & 0 & 0 & C_{64} & C_{65} & C_{66}
\end{array}\right]\left\{\begin{array}{c}
\varepsilon_{\rho \rho} \\
\varepsilon_{\phi \phi} \\
\varepsilon_{\theta \theta} \\
\varepsilon_{\rho \theta} \\
\varepsilon_{\rho \phi} \\
\varepsilon_{\theta \phi}
\end{array}\right\},
$$

onde $\mathrm{C}_{\alpha \beta}=\mathrm{C}_{\beta \alpha}, \alpha, \beta=1, \ldots, 6$ são as constantes elásticas do material. 
Para que a matriz $\left[\mathrm{C}_{\alpha \beta}\right]$ seja positiva definida ${ }^{1}$, as constantes elásticas da relação (3.14) devem satisfazer as seguintes desigualdades:

$$
\mathrm{C}_{11}>0, \mathrm{C}_{22}>0,\left|\mathrm{C}_{23}\right|<\mathrm{C}_{22}, \mathrm{C}_{11}>\frac{2 \mathrm{C}_{12}^{2}}{\mathrm{C}_{22}+\mathrm{C}_{23}}
$$

Assume-se que o campo de deslocamentos é radialmente simétrico em relação ao centro da esfera, de modo que as componentes de deslocamento de um ponto da esfera no sistema de coordenadas esféricas são dadas por

$$
\mathrm{u}_{\rho}(\rho, \phi, \theta)=\mathrm{u}(\rho), \quad \mathrm{u}_{\phi}=\mathrm{u}_{\theta}=0
$$

Esta consideração reduz o problema original, tridimensional, a um problema unidimensional. Substituindo-se (3.16) nas expressões (3.11), obtêm-se

$$
\varepsilon_{\rho \rho}=\frac{\mathrm{du}}{\mathrm{d} \rho}, \quad \varepsilon_{\phi \phi}=\varepsilon_{\theta \theta}=\frac{\mathrm{u}}{\rho}, \quad \varepsilon_{\theta \phi}=\varepsilon_{\rho \theta}=\varepsilon_{\phi \rho}=0
$$

Utilizando-se (3.17), segue de (3.14) que

$$
\sigma_{\rho \rho}=C_{11} \frac{d u}{d \rho}+2 C_{12} \frac{u}{\rho}, \sigma_{\theta \theta}=\sigma_{\phi \phi}=C_{12} \frac{d u}{d \rho}+\left(C_{22}+C_{23}\right) \frac{u}{\rho}
$$

A consideração de deformações radialmente simétricas implica que $\sigma_{\theta \phi}=\sigma_{\phi \rho}=\sigma_{\rho \theta}=0$ e a consideração de material esfericamente uniforme implica que

\footnotetext{
${ }^{1}$ Ting (1996) apresenta as condições necessárias para que a matriz 6x6 em (3.14) seja positiva definida.
} 
$\sigma_{\theta \theta}=\sigma_{\phi \phi}$, o que reduz as equações de equilíbrio (3.10) a uma única equação não trivial dada por

$$
\frac{\mathrm{d} \sigma_{\rho \rho}}{\mathrm{d} \rho}+\frac{2}{\rho}\left(\sigma_{\rho \rho}-\sigma_{\theta \theta}\right)=0 \text { em } \Omega
$$

Substituindo-se as expressões de $\sigma_{\rho \rho}$ e $\sigma_{\theta \theta}$ dadas por (3.18) em (3.19) chega-se à equação diferencial do problema da esfera anisotrópica sob pressão radial, que é dada por

$$
\frac{d^{2} u}{d \rho^{2}}+\frac{2}{\rho} \frac{d u}{d \rho}-2 \gamma \frac{u}{\rho^{2}}=0, \quad 0<\rho<\rho_{e},
$$

onde

$$
\gamma=\frac{\mathrm{C}_{22}+\mathrm{C}_{23}-\mathrm{C}_{12}}{\mathrm{C}_{11}}
$$

As condições de contorno essencial e natural deste problema são dadas, respectivamente, por

$$
u(0)=0, \quad \sigma_{\rho \rho}\left(\rho_{\mathrm{e}}\right)=-p
$$

onde a tensão radial $\sigma_{\rho \rho}$ é dada por (3.18a). 


\subsection{OBTENÇÃO DA SOLUÇÃO EXATA DO PROBLEMA DA ESFERA}

A solução geral da equação diferencial (3.20) é da forma

$$
u(\rho)=\alpha^{+} \rho^{\lambda^{+}}+\alpha^{-} \rho^{\lambda^{-}},
$$

onde $\lambda^{+}, \lambda^{-}, \alpha^{+}$e $\alpha^{-}$são constantes a determinar a partir das condições de contorno (3.22) do problema. Derivando-se a solução (3.23) duas vezes e substituindo-se o resultado em (3.20), tem-se que

$$
\lambda^{ \pm} \equiv \frac{1}{2}(-1 \pm 3 \kappa), \kappa \equiv \frac{1}{3} \sqrt{1+8 \gamma}
$$

onde $\gamma$ é dado por (3.21).

Utilizando-se a condição $\mathrm{u}(0)=0$, obtém-se que $\alpha^{-}=0$. Utilizando-se agora a condição de contorno natural (3.22b), determina-se o valor da constante $\alpha^{+}$, que é dada por

$$
\alpha^{+}=-q \rho_{e}{ }^{1-\lambda^{+}}, q \equiv \frac{p}{C_{11} \lambda^{+}+2 C_{12}}
$$

Portanto, a solução exata do problema da esfera, considerando-se deformações radialmente simétricas e material esfericamente uniforme, é dada por

$$
u(\rho)=-q \rho_{e}\left(\frac{\rho}{\rho_{e}}\right)^{\lambda^{+}}
$$


Utilizando-se (3.26) juntamente com (3.25), (3.24) e (3.21), obtém-se de (3.18) que as tensões $\sigma_{\rho \rho}$ e $\sigma_{\theta \theta}=\sigma_{\phi \phi}$ são dadas por

$$
\sigma_{\rho \rho}=-p\left(\frac{\rho}{\rho_{\mathrm{e}}}\right)^{3(\kappa-1) / 2}, \sigma_{\theta \theta}=\sigma_{\phi \phi}=-\mathrm{p}\left(\frac{1+3 \kappa}{4}\right)\left(\frac{\rho}{\rho_{\mathrm{e}}}\right)^{3(\kappa-1) / 2}
$$

Observa-se por fim que

$$
\frac{\sigma_{\rho \rho}}{\sigma_{\theta \theta}}=\frac{4}{1+3 \kappa}
$$

ou seja, as tensões são proporcionais.

\subsection{ANÁLISE DA SOLUÇÃO}

Utilizando-se a restrição (3.15d) juntamente com as definições (3.21) e (3.24), chegase a

$$
\left(\mathrm{C}_{11} \lambda^{+}+2 \mathrm{C}_{12}\right)\left(\mathrm{C}_{11} \lambda^{-}+2 \mathrm{C}_{12}\right)=-2\left[\mathrm{C}_{11}\left(\mathrm{C}_{22}+\mathrm{C}_{23}\right)-2 \mathrm{C}_{12}^{2}\right]<0,
$$

o que implica que

$$
\mathrm{C}_{11} \lambda^{+}+2 \mathrm{C}_{12}>0,-\left(\mathrm{C}_{11} \lambda^{-}+2 \mathrm{C}_{12}\right)<0 .
$$


Reescrevendo-se (3.29) na forma alternativa

$$
-\left(\frac{\mathrm{C}_{11}}{2}\right)^{2}\{[3 \kappa+(4 \eta-1)][3 \kappa-(4 \eta-1)]\}<0,
$$

conclui-se que $\kappa$ é uma constante positiva e diferente de zero,

$$
0<\kappa<\infty .
$$

\subsubsection{CAMPO DE DESLOCAMENTO}

Analisando-se a expressão (3.23) juntamente com (3.24), observa-se que o problema (3.20)-(3.22) não possui solução para $\kappa \leq 1 / 3$. Neste trabalho, assume-se que $\kappa>1 / 3$, de modo que a solução (3.26) satisfaz a condição de compatibilidade $u(0)=0$ e a condição de contorno $\sigma_{\rho \rho}\left(\rho_{\mathrm{e}}\right)=-\mathrm{p}<0$.

Além disto, observa-se de (3.25b) que para $\mathrm{p}>0$ e para a condição (3.29), q >0, implicando que o deslocamento das partículas da esfera descrito por (3.26) acontece no sentido do raio para o centro, ou seja, a esfera contrai.

Em particular, se $-\mathrm{u}\left(\rho_{\mathrm{e}}\right)=\rho_{\mathrm{e}}$ observa-se de (3.26) que $\mathrm{q}=1$, ou seja, para $\mathrm{q}=1 \mathrm{o}$ encurtamento em $\rho_{\mathrm{e}}$ é igual ao próprio raio, significando que a superfície externa da esfera é mapeada em um único ponto no seu centro. Como este mapeamento não é fisicamente admissível, devem ser impostas restrições nos valores de $\mathrm{p}$ que podem ser aplicados na superfície da esfera. Para $q>1$, observa-se que o deslocamento em $\rho_{\mathrm{e}}$ é maior que o raio, $-u\left(\rho_{e}\right)>\rho_{e}$, implicando que o deslocamento em $\rho_{e}$, para $\kappa \geq 1$, deve satisfazer a 
desigualdade

$$
\frac{-\mathrm{u}\left(\rho_{\mathrm{e}}\right)}{\rho_{\mathrm{e}}}=\mathrm{q}<1
$$

Com (3.33) e (3.25b) encontram-se os valores de $\mathrm{p}$ que podem ser aplicados na superfície da esfera para que isso seja evitado. Estes valores devem satisfazer a desigualdade

$$
\mathrm{C}_{11} \lambda^{+}+2 \mathrm{C}_{12}>\mathrm{p}
$$

Reescrevendo a expressão (3.26) para $\rho \in\left(0, \rho_{\mathrm{e}}\right)$, obtém-se:

$$
\frac{u(\rho)}{\rho}=-q\left(\frac{\rho}{\rho_{e}}\right)^{3(\kappa-1) / 2},
$$

de onde se observa que para $0<\rho<\rho_{\mathrm{e}}$ e $\kappa \geq 1$,

$$
-\frac{u(\rho)}{\rho}<q
$$

\subsubsection{CAMPO DE TENSÃO}

Observa-se de (3.27) que se $\kappa>1$ as tensões $\sigma_{\rho \rho}$ e $\sigma_{\theta \theta}=\sigma_{\phi \phi}$ são nulas no centro da esfera $(\rho=0)$. Para qualquer $p \neq 0$ e para qualquer $0<\kappa<1$, observa-se que as tensões, $\sigma_{\rho \rho}$ e $\sigma_{\theta \theta}=\sigma_{\phi \phi}$ são singulares, ou seja, tendem ao infinito no centro da esfera $(\rho=0)$ e que se 
$\kappa=1$ as tensões são constantes e iguais, $\sigma_{\rho \rho}=\sigma_{\theta \theta}=\sigma_{\phi \phi}$, correspondendo ao problema da esfera isotrópica.

\subsubsection{CAMPO DE DEFORMAÇÃO}

Seja $\Omega_{R}$ a configuração de referência natural indeformada de um corpo, $\Omega_{D}$ a configuração atual deformada e f um mapeamento, chamado função mudança de configuração, que leva os pontos $\mathrm{x} \in \Omega_{\mathrm{R}}$ a pontos $\mathrm{X} \in \Omega_{\mathrm{D}}$ (Fig. 3.8). Um mapeamento $\mathrm{f}$ é injetivo quando mapeia um ponto x da configuração de referência $\Omega_{\mathrm{R}}$ em um, e apenas um ponto $\mathrm{X}$ da configuração deformada $\Omega_{\mathrm{D}}$.

Do ponto $\mathrm{x} \in \Omega_{\mathrm{R}}$ emanam os vetores $\mathrm{dx}$, $\mathrm{dy}, \mathrm{dz}$ que são mapeados por $\mathrm{f}$ nos vetores $\mathrm{dX}, \mathrm{dY}, \mathrm{dZ}$ que emanam do ponto $\mathrm{X} \in \Omega_{\mathrm{D}}$ (Fig. 3.8).

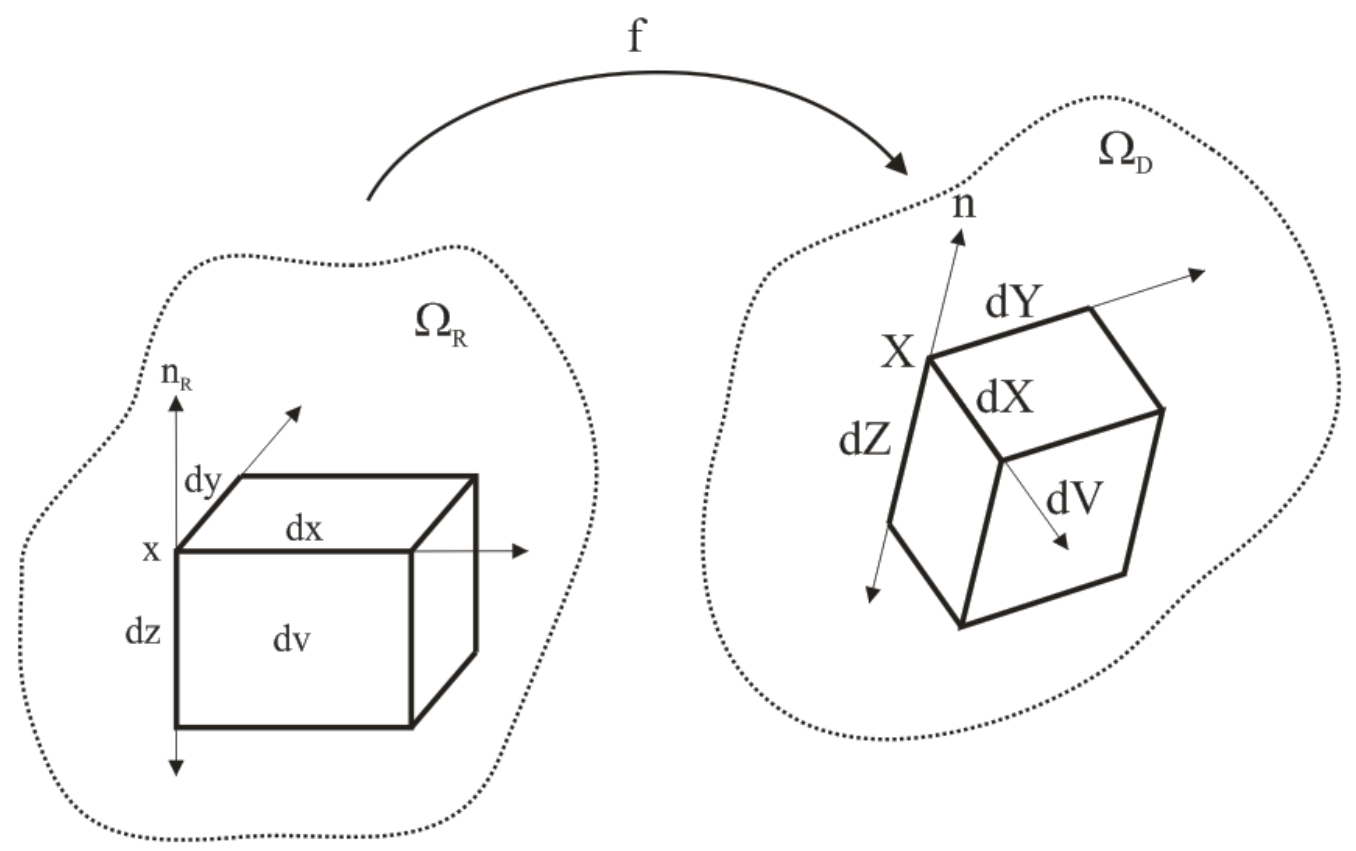

Figura 3.8. Configuração de referência e atual de um corpo. 
Os vetores da configuração de referência $\mathrm{dx}$, dy, $\mathrm{dz}$ relacionam-se aos vetores da configuração deformada $\mathrm{dX}, \mathrm{dY}, \mathrm{dZ}$ por

$$
\mathrm{dx}=\nabla \mathrm{f} d X, \mathrm{dy}=\nabla \mathrm{f} \mathrm{dY}, \mathrm{dz}=\nabla \mathrm{f} \mathrm{dZ}
$$

Os elementos de volume $d v \in \Omega_{R}$ e $d V \in \Omega_{D}$ estão relacionados por

$$
\mathrm{dV}=(\operatorname{det} \nabla f) \mathrm{dv}
$$

De (3.38) observa-se que o determinante do gradiente da função mudança de configuração, det $\nabla f$, representa, localmente, o volume de um corpo depois da deformação por unidade de volume do corpo na configuração de referência natural indeformada (GURTIN, 1981).

Desta forma, é razoável assumir que $\operatorname{det} \nabla f \neq 0$, pois se $\operatorname{det} \nabla f=0$, ou seja, se o volume $\mathrm{dV}$ desaparece após a mudança de configuração significa dizer que um corpo tridimensional foi mapeado por $\mathrm{f}$, em um plano, uma reta, ou um ponto, sendo todas estas possibilidades inadmissíveis do ponto de vista físico. Uma vez que det $\nabla f=1$ para $f(x)=x$, deseja-se que

$$
\operatorname{det} \nabla f>0 \text {. }
$$

A desigualdade (3.39) é a restrição que deve ser satisfeita para que um mapeamento seja localmente injetivo.

O gradiente da função mudança de configuração na expressão (3.39), $\nabla f$, mede alterações no tamanho e na forma dos elementos de um corpo quando ele passa da 
configuração de referência $\Omega_{\mathrm{R}}$ para a configuração deformada $\Omega_{\mathrm{D}}$ e é definido por

$$
\nabla \mathrm{f}=\mathrm{I}+\nabla \mathrm{u}
$$

onde I é o tensor identidade e $\nabla \mathbf{u}$ é o gradiente de deslocamento.

Analisando-se a solução (3.26) reescrita na forma alternativa

$$
-u(\rho)=\rho \frac{q}{\left(\frac{\rho}{\rho_{\mathrm{e}}}\right)^{3(1-\kappa) / 2}}
$$

observa-se que para $\kappa<1$ e se $q>\left(\rho / \rho_{\mathrm{e}}\right)^{3(1-\kappa) / 2}$, que $\frac{\mathrm{q}}{\left(\rho / \rho_{\mathrm{e}}\right)^{3(1-\kappa) / 2}}>1$, implicando que $-\mathrm{u}(\rho)>\rho$, ou seja, existe uma região central da esfera delimitada por

$$
0<\left(\frac{\rho}{\rho_{\mathrm{e}}}\right)^{3(1-\kappa) / 2}<\mathrm{q},
$$

para a qual o campo de deformação não é localmente injetivo. Então, para $\kappa<1$ existe um raio $\rho_{c}$ que delimita esta região central onde o material se auto-intersecciona. Para determinálo, faz-se $u\left(\rho_{c}\right)=-\rho_{c}$ em (3.26), de onde se obtém que

$$
\left(\frac{\rho_{\mathrm{c}}}{\rho_{\mathrm{e}}}\right)^{3(1-\kappa) / 2}=\mathrm{q} .
$$


Por fim, observa-se que se

$$
\left(\frac{\rho}{\rho_{\mathrm{e}}}\right)^{3(1-\kappa) / 2}<\lambda^{+} \mathrm{q}
$$

o determinante do gradiente de deformação, dado por

$$
\operatorname{det} \nabla f=\left[1-\lambda^{+} \mathrm{q}\left(\frac{\rho}{\rho_{\mathrm{e}}}\right)^{3(\kappa-1) / 2}\right]\left[1-\mathrm{q}\left(\frac{\rho}{\rho_{\mathrm{e}}}\right)^{3(\kappa-1) / 2}\right]^{2},
$$

é negativo, ou seja, para $\kappa<1$ a teoria da elasticidade linear clássica fornece uma solução para o problema da esfera que não é fisicamente admissível. 


\subsection{CONSIDERAÇÕES INICIAIS}

O modelo matemático descrito pelas equações de equilíbrio (3.10), relações deformação-deslocamento (3.11) e equações constitutivas (3.12) acrescidas das condições de contorno em deslocamento $\overline{\mathrm{u}}(\mathrm{x})$ e das condições de contorno em carregamento $\overline{\mathrm{t}}(\mathrm{x})$ é conhecido como Problema de Valor de Contorno (PVC) da elasticidade linear clássica. Como estas relações devem ser satisfeitas para qualquer ponto do domínio do problema estudado, o modelo também é conhecido como formulação forte ou local.

Uma vez conhecida a formulação forte de um problema, é possível escrevê-la em uma forma alternativa, dita variacional, mediante integração ponderada da equação, ou, conjunto de equações que a constituem. Integrando-se por partes a integral ponderada da formulação variacional, obtém-se a formulação fraca. A partir da formulação fraca é possível obter soluções aproximadas para o problema de valor de contorno que a originou.

Observa-se, porém, que a obtenção da formulação fraca da forma como exposto acima depende do conhecimento da equação diferencial que descreve o problema, o que pode se tornar um inconveniente no estudo de problemas mais complexos. No entanto, é possível 
gerar a forma fraca de um problema sem conhecer a equação diferencial que o descreve empregando-se princípios variacionais fundamentados em conceitos de energia, ou, através do Princípio dos Trabalhos Virtuais (CIARLET, 1979).

\subsection{PROBLEMA DE MINIMIZAÇÃO}

Seja V um espaço vetorial normado, a[ $\cdot \cdot]: \mathrm{V} x \mathrm{~V} \rightarrow \mathrm{R}$ uma forma bilinear contínua, $1: \mathrm{V} \rightarrow \mathrm{R}$ uma forma linear contínua e $\mathrm{U}$ um subconjunto não vazio do espaço $\mathrm{V}$. Um problema de minimização consiste em encontrar $\mathrm{u} \in \mathrm{U}$ tal que

$$
\mathrm{J}(\mathrm{u})=\inf _{\mathrm{v} \in \mathrm{U}} \mathrm{J}(\mathrm{v})
$$

onde o funcional $\mathrm{J}: \mathrm{V} \rightarrow \mathrm{R}$ é definido por

$$
\mathrm{J}: \mathrm{v} \in \mathrm{V} \rightarrow \mathrm{J}(\mathrm{v})=\frac{1}{2} \mathrm{a}[\mathrm{v}, \mathrm{v}]-\mathrm{l}[\mathrm{v}]
$$

Este problema pode ser obtido empregando-se conceitos de energia e corresponde ao problema da mínima energia potencial em mecânica (CIARLET, 1979).

\subsection{PRINCÍPIO DA MÍNIMA ENERGIA POTENCIAL}

O princípio da mínima energia potencial diz que de todos os possíveis deslocamentos que satisfazem as condições de contorno de um sistema, aquele que corresponde à configuração de equilíbrio estável faz da energia potencial um mínimo relativo, ou seja, $\mathrm{J}=0$ 
(ODEN, 1967; SOKOLNIKOFF, 1956).

A presença de energia em um sistema está relacionada à capacidade de realização de trabalho. Para que um sistema realize trabalho é necessário que haja deslocamento das partículas que o constituem e força, ou, componente de força, na direção deste deslocamento. Pode-se então definir trabalho como uma medida das transformações de energia causadas por uma força, ou, conjunto de forças sobre um sistema. Assim, a energia potencial das forças externas relacionada ao trabalho realizado pelas forças externas pode ser convertida em energia de deformação relacionada à capacidade das forças internas de realizar trabalho e vice-versa (ODEN, 1967).

Na expressão (4.2), a forma bilinear $\mathrm{a}[\mathrm{v}, \mathrm{v}] / 2$ corresponde à energia de deformação relacionada ao trabalho realizado pelas forças internas que atuam em um sistema e é dada, por

$$
\frac{1}{2} \mathrm{a}[\mathrm{v}, \mathrm{v}]=\int_{\Omega}\left(\int_{0}^{\varepsilon_{\mathrm{ij}}} \sigma_{\mathrm{ij}} \mathrm{d} \varepsilon_{\mathrm{ij}}\right) \mathrm{d} \Omega, \quad \mathrm{i}, \mathrm{j}=1,2,3,
$$

onde $\sigma_{\mathrm{ij}}$ são as componentes de tensão e $\varepsilon_{\mathrm{ij}}$ são as componentes de deformação. A expressão (4.3) pode ser utilizada para calcular a energia de deformação em qualquer estrutura elástica, linear ou não-linear, e a integração é realizada do estado inicial ao estado final de deformação do corpo.

A forma linear $1[\mathrm{v}]$ na expressão (4.2) corresponde ao trabalho realizado pelas forças externas e é dada por

$$
1[v]=\int_{\Omega} b^{\mathrm{T}} \mathrm{vd} \Omega+\int_{\partial_{2} \Omega} \overline{\mathrm{t}}^{\mathrm{T}} \mathrm{v} \mathrm{d} S+\sum_{\mathrm{i}=1}^{\mathrm{n}} \overline{\mathrm{q}}_{\mathrm{i}} \mathrm{v}_{\mathrm{i}},
$$


onde $\overline{\mathrm{q}}$ são forças concentradas que podem atuar no sistema.

Define-se a energia potencial total de um sistema como a soma das energias de deformação interna e das forças externas (ODEN, 1967; TAUCHERT, 1974).

\subsection{PROBLEMA VARIACIONAL}

Utilizando as definições de $\mathrm{V}, \mathrm{a}[\cdot, \cdot]: \mathrm{V} \times \mathrm{V} \rightarrow \mathrm{R}$ e $1: \mathrm{V} \rightarrow \mathrm{R}$ introduzidas na Seção 4.2, tem-se que um problema variacional consiste em encontrar $\mathrm{u} \in \mathrm{V}$ tal que

$$
\mathrm{a}[\mathrm{u}, \mathrm{v}]=1[\mathrm{v}], \quad \forall \mathrm{v} \in \mathrm{V},
$$

Este problema variacional pode ser obtido a partir da integração ponderada da equação diferencial que descreve o problema, quando esta é conhecida, ou, empregando-se o Princípio dos Trabalhos Virtuais.

\subsection{PRINCÍPIO DOS TRABALHOS VIRTUAIS}

O Princípio dos Trabalhos Virtuais (PTV) diz que se um corpo está em equilíbrio e permanece em equilíbrio enquanto é submetido a um deslocamento virtual, o trabalho virtual externo realizado pelas forças externas atuantes no corpo é igual ao trabalho virtual interno realizado pelas forças internas (ODEN, 1967).

A forma bilinear $\mathrm{a}[\mathrm{u}, \mathrm{v}]$ em (4.5) corresponde ao trabalho virtual realizado pelas forças internas e a forma linear $1[\mathrm{v}]$ corresponde ao trabalho virtual realizado pelas forças externas, que são dadas, respectivamente, pelas expressões (4.3) e (4.4). Pode-se mostrar que o problema de minimização e o problema variacional são equivalentes (JOHNSON, 1987). 
Em particular, é possível mostrar que a formulação do PTV pode ser obtida a partir da expressão da energia total do sistema empregando-se o princípio variacional que diz que na configuração de equilíbrio a primeira variação do potencial possui valor estacionário, ou seja, $\delta \mathrm{J}=0$.

\subsection{ENERGIA DE DEFORMAÇÃO DA ESFERA ELÁSTICA ANISOTRÓPICA}

A partir da expressão (4.3) é possível escrever a expressão da energia de deformação da esfera em coordenadas esféricas como

$$
\mathrm{a}[\mathrm{v}, \mathrm{v}]=\int_{\Omega}\left(\sigma_{\rho \rho} \mathrm{e}_{\rho \rho}+\sigma_{\phi \phi} \mathrm{e}_{\phi \phi}+\sigma_{\theta \theta} \mathrm{e}_{\theta \theta}+\sigma_{\rho \phi} \mathrm{e}_{\rho \phi}+\sigma_{\rho \theta} \mathrm{e}_{\rho \theta}+\sigma_{\theta \phi} \mathrm{e}_{\theta \phi}\right) \mathrm{d} \Omega
$$

Lembrando-se da Seção 3.5 que $\sigma_{\theta \phi}=\sigma_{\phi \rho}=\sigma_{\rho \theta}=0$ e substituindo-se as expressões de $\sigma_{\rho \rho}, \sigma_{\phi \phi}$ e $\sigma_{\theta \theta}$ dadas na Eq. (3.18) na expressão (4.6), tem-se

$$
a[v, v]=\int_{\Omega}\left\{\left(C_{11} \frac{d v}{d \rho}+2 C_{12} \frac{v}{\rho}\right) \frac{d v}{d \rho}+2\left[C_{21} \frac{d v}{d \rho}+\left(C_{22}+C_{23}\right) \frac{v}{\rho}\right] \frac{v}{\rho}\right\} d \Omega .
$$

Substituindo-se $\mathrm{d} \Omega=\rho^{2} \operatorname{sen} \theta \mathrm{d} \theta \mathrm{d} \phi \mathrm{d} \rho$ e rearranjando-se os termos no integrando de (4.7), tem-se que

$$
\mathrm{a}[\mathrm{v}, \mathrm{v}]=\int_{0}^{\rho_{\mathrm{e}}} \int_{0}^{2 \pi} \int_{0}^{\pi}\left[\mathrm{C}_{11} \frac{\mathrm{dv} \mathrm{v}^{2}}{\mathrm{~d} \rho}+4 \mathrm{C}_{12} \frac{\mathrm{v}}{\rho} \frac{\mathrm{dv}}{\mathrm{d} \rho}+2\left(\mathrm{C}_{22}+\mathrm{C}_{23}\right) \frac{\mathrm{v}^{2}}{\rho^{2}}\right] \rho^{2} \operatorname{sen} \theta \mathrm{d} \theta \mathrm{d} \phi \mathrm{d} \rho
$$

Uma vez que v só depende de $\rho$, segue de (4.8) que 


$$
\mathrm{a}[\mathrm{v}, \mathrm{v}]=4 \pi \int_{0}^{\rho_{\mathrm{e}}}\left[\mathrm{C}_{11}\left(\frac{\mathrm{dv}}{\mathrm{d} \rho}\right)^{2} \rho^{2}+2 \mathrm{C}_{12} \frac{\mathrm{dv} \mathrm{v}^{2}}{\mathrm{~d} \rho} \rho+2\left(\mathrm{C}_{22}+\mathrm{C}_{23}\right) \mathrm{v}^{2}\right] \mathrm{d} \rho
$$

\subsection{ENERGIA POTENCIAL DAS FORÇAS EXTERNAS QUE ATUAM NA ESFERA ELÁSTICA ANISOTRÓPICA}

A energia potencial das forças externas que atuam na esfera é dada por

$$
1[v]=\int_{\partial_{2} \Omega} p v\left(\rho_{\mathrm{e}}\right) \mathrm{d} S
$$

onde $\mathrm{p}$ é a força compressiva uniformemente distribuída sobre a superfície externa da esfera, $\mathrm{v}\left(\rho_{\mathrm{e}}\right)$ é o deslocamento na direção da força dos pontos materiais pertencentes à superfície externa da esfera e dS é o elemento diferencial da superfície externa da esfera.

Sabendo-se que $\mathrm{dS}=\rho_{\mathrm{e}}^{2} \operatorname{sen} \theta \mathrm{d} \theta \mathrm{d} \phi$, a expressão (4.10) pode ser reescrita como

$$
\mathbb{1}[\mathrm{v}]=\int_{0}^{2 \pi} \int_{0}^{\pi} \mathrm{pv}\left(\rho_{\mathrm{e}}\right) \rho_{\mathrm{e}}^{2} \operatorname{sen} \theta \mathrm{d} \theta \mathrm{d} \phi
$$

Uma vez que $\operatorname{pv}\left(\rho_{\mathrm{e}}\right) \rho_{\mathrm{e}}^{2}$ é constante, segue de (4.11) que:

$$
1[v]=4 \pi p v\left(\rho_{e}\right) \rho_{e}^{2}
$$

Portanto, segue de (4.2), (4.9) e (4.12) que a energia potencial total $\mathrm{J}$ da esfera é dada por: 


$$
J=2 \pi \int_{0}^{\rho_{\mathrm{e}}}\left[\mathrm{C}_{11}\left(\frac{d v}{d \rho}\right)^{2} \rho^{2}+2 C_{12}\left(\frac{d v^{2}}{d \rho}\right) \rho+2\left(C_{22}+C_{23}\right) v^{2}\right] d \rho+4 \pi p v\left(\rho_{e}\right) \rho_{e}{ }^{2}
$$




\subsection{CONSIDERAÇÕES INICIAIS}

Fosdick e Royer-Cargfagni (2001) propõem minimizar o funcional quadrático da energia potencial total submetido à restrição da injetividade det $\nabla f>0$ e mostram a existência de solução para o problema assim formulado no caso plano.

O problema consiste em

$$
\min _{\mathrm{v} \in \mathrm{A}_{\varepsilon}} \mathrm{J}[\mathrm{v}], \mathrm{J}[\mathrm{v}] \equiv \frac{1}{2} \mathrm{a}[\mathrm{v}, \mathrm{v}]-1[\mathrm{v}]
$$

onde $\mathrm{J}[\mathrm{v}]$ é o funcional quadrático da energia potencial total, $\mathrm{a}[\mathrm{v}, \mathrm{v}]$ é a forma bilinear e $1[\mathrm{v}]$ é a forma linear, dadas, respectivamente, por

$$
\mathrm{a}[\mathrm{v}, \mathrm{v}] \equiv \int_{\Omega} \mathrm{C}[\mathrm{E}] \cdot[\mathrm{E}] \mathrm{dx}, 1[\mathrm{v}] \equiv \int_{\Omega} \mathrm{b} \cdot \mathrm{vdx}+\int_{\partial_{2} \Omega} \overline{\mathrm{t}} \cdot \mathrm{vdx}
$$

onde $\mathrm{C}=\mathrm{C}(\mathrm{x})$ é o tensor elasticidade e $\mathrm{E} \equiv\left[\nabla \mathrm{v}+(\nabla \mathrm{v})^{\mathrm{T}}\right] / 2$ é o tensor das deformações 
infinitesimais.

Em (5.1), o conjunto $A_{\varepsilon}$, definido por

$$
\mathrm{A}_{\varepsilon} \equiv\left\{\mathrm{v}: \mathrm{W}^{1,2}(\Omega) \rightarrow \mathrm{R}^{3} \mid \operatorname{det}(1+\nabla \mathrm{v}) \geq \varepsilon>0, \mathrm{v}=0 \text { sobre } \partial_{1} \Omega\right\}
$$

é uma classe de campos de deslocamento admissível que satisfazem a restrição da injetividade $\operatorname{det}(1+\nabla v) \geq \varepsilon>0$, onde $\varepsilon$ é um valor positivo suficientemente pequeno.

\subsection{CONDIÇÕES NECESSÁRIAS PARA EXISTÊNCIA DE MÍNIMO}

Apresentam-se as condições necessárias de primeira ordem para a existência de um mínimo $\mathrm{u} \in \mathrm{A}_{\varepsilon}$ de J[·] em (5.1) deduzidas por Fosdick e Royer-Carfagni (2001).

Considera-se o problema descrito no segundo parágrafo da Seção 3.1 e seja um conjunto de deslocamentos admissíveis definido por:

$$
\mathrm{V} \equiv\left\{\mathrm{v}: \mathrm{W}^{1,2}(\Omega) \rightarrow \mathrm{R}^{3}: \mathrm{v}=0 \text { sobre } \partial_{1} \Omega\right\}
$$

A primeira variação de $J[\cdot]$ em função de u é dada por:

$$
\lim _{\tau \rightarrow 0} \frac{\mathrm{J}[\mathrm{u}+\tau \mathrm{u}]-\mathrm{J}[\mathrm{u}]}{\tau} \equiv\langle\mathrm{DJ}[\mathrm{u}], \mathrm{v}\rangle \equiv \mathrm{a}[\mathrm{u}, \mathrm{v}]-\mathrm{l}[\mathrm{v}], \quad \forall \mathrm{v} \in \mathrm{V},
$$

onde $\mathrm{a}[\cdot \cdot \cdot]$ e $1[\cdot]$ são, respectivamente, as formas bilinear e linear do problema de minimização (5.1) e são dadas por (5.2). 
Os autores mostram que existe um campo escalar de multiplicadores de Lagrange $\lambda: \mathrm{L}^{2}(\Omega) \rightarrow \mathrm{R}$, de forma que a primeira variação de (5.5) pode ser representada equivalentemente por

$$
\langle\mathrm{DJ}[\mathrm{u}], \mathrm{v}\rangle \equiv \int_{\mathrm{B}} \lambda(\operatorname{cof} \nabla \mathrm{f}) \cdot \nabla \mathrm{vdx}, \quad \forall \mathrm{v} \in \mathrm{V},
$$

onde $\operatorname{cof} \nabla f=(\operatorname{det} \nabla f)\left[(\nabla f)^{-1}\right]^{T}$ é o cofator do gradiente da função mudança de configuração.

Definindo-se as regiões onde a restrição da injetividade está ativa e não ativa, respectivamente, por:

$$
\begin{aligned}
& \Omega_{=} \equiv \operatorname{int}[\{x \in \Omega: \operatorname{det} \nabla f=\varepsilon\}], \\
& \Omega_{>} \equiv \operatorname{int}[\{x \in \Omega: \operatorname{det} \nabla f>\varepsilon\}],
\end{aligned}
$$

onde int[·] representa o interior de um conjunto, as condições necessárias de primeira ordem são dadas pelas equações de Euler-Lagrange definidas em cada região e pela condição de continuidade entre as duas regiões que devem ser satisfeitas para que um mínimo $u \in A_{\varepsilon}$ exista. Estas condições são apresentadas a seguir.

\subsubsection{EQUAÇÕES DE EULER-LAGRANGE}

As equações de Euler-Lagrange definidas para a região $\Omega_{>}$, onde a restrição da injetividade não está ativa, e para a região $\Omega_{=}$, onde a restrição está ativa, são dadas, respectivamente, por: 


$$
\begin{aligned}
& \operatorname{Div}+\mathrm{b}=0 \quad \text { em } \Omega_{>}, \\
& \operatorname{Div}\left(\mathrm{T}-\varepsilon \lambda(\nabla \mathrm{f})^{-\mathrm{T}}\right)+\mathrm{b}=0, \quad \lambda \geq 0, \quad \text { em } \Omega_{=},
\end{aligned}
$$

onde $\mathrm{T}$ é o tensor tensão que em relação a uma base esférica ortonormal $\left(\mathrm{e}_{\rho}, \mathrm{e}_{\phi}, \mathrm{e}_{\theta}\right)$ é dado por

$$
\begin{gathered}
\mathrm{T}=\sigma_{\rho \rho} \mathrm{e}_{\rho} \otimes \mathrm{e}_{\rho}+\sigma_{\phi \phi} \mathrm{e}_{\phi} \otimes \mathrm{e}_{\phi}+\sigma_{\theta \theta} \mathrm{e}_{\theta} \otimes \mathrm{e}_{\theta}+\sigma_{\rho \phi}\left(\mathrm{e}_{\rho} \otimes \mathrm{e}_{\phi}+\mathrm{e}_{\phi} \otimes \mathrm{e}_{\rho}\right)+ \\
\sigma_{\rho \theta}\left(\mathrm{e}_{\rho} \otimes \mathrm{e}_{\theta}+\mathrm{e}_{\theta} \otimes \mathrm{e}_{\rho}\right)+\sigma_{\theta \phi}\left(\mathrm{e}_{\theta} \otimes \mathrm{e}_{\phi}+\mathrm{e}_{\phi} \otimes \mathrm{e}_{\theta}\right)
\end{gathered}
$$

As condições de contorno são dadas por

$$
\begin{aligned}
& \text { Tn }=\overline{\mathrm{t}} \quad \text { sobre } \partial \Omega_{>} \cap \partial_{2} \Omega, \\
& \left(\mathrm{T}-\varepsilon \lambda(\nabla \mathrm{f})^{-\mathrm{T}}\right) \mathrm{n}=\overline{\mathrm{t}} \quad \text { sobre } \partial \Omega_{=} \cap \partial_{2} \Omega,
\end{aligned}
$$

onde n é a normal unitária a $\partial_{2} \Omega$.

\subsubsection{CONDIÇÃO DE CONTINUIDADE}

Seja $\sum \equiv \bar{\Omega}_{>} \cap \bar{\Omega}_{=}$a interface suficientemente suave entre as regiões $\Omega_{=}$e $\Omega_{>}$. A condição de continuidade das tensões na direção normal a esta interface é dada por

$$
\left.\left(\mathrm{T}-\varepsilon \lambda(\nabla \mathrm{f})^{-\mathrm{T}}\right)\right|_{\sum \cap \bar{\Omega}_{=}} \mathrm{n}=\left.\mathrm{T}\right|_{\sum \cap \bar{\Omega}_{>}} \mathrm{n},
$$

onde $\mathrm{n}$ é a normal unitária à interface $\sum$ e os termos $(\cdot) \mid \sum \cap \bar{\Omega}_{=}$e $(\cdot) \mid \sum \cap \bar{\Omega}_{>} \quad$ são avaliados 
no limite, ou seja, à medida que se aproxima da interface $\sum$ pelo interior de $\Omega_{=}$e $\Omega_{>}$, respectivamente.

O termo $\mathrm{T}-\varepsilon \lambda(\nabla f)^{-\mathrm{T}}$ presente nas equações (5.10), (5.13) e (5.14) representa a tensão total que atua em $\Omega_{=}$e o multiplicador de Lagrange $\lambda$ pode ser interpretado como uma pressão reativa que surge devido à imposição da restrição $\operatorname{det} \nabla f=\varepsilon$ em $\Omega_{=}$.

\subsection{O PROBLEMA DA ESFERA COM RESTRIÇÃO}

Lembra-se da Seção 3.7.3 que a solução do problema da esfera anisotrópica apresenta um campo de deformação que não é localmente injetivo em uma região próxima ao centro quando o parâmetro material $\kappa$ é menor que 1 , onde $\kappa$ é dado por $(3.24 b)$ juntamente com (3.21). Aguiar (2006b) utiliza as condições necessárias de primeira ordem (5.9 - 5.14) para obter um campo de deslocamento admissível em $\mathrm{A}_{\varepsilon}$ para $\kappa \in(0,1)$. Esta solução é apresentada no presente capítulo.

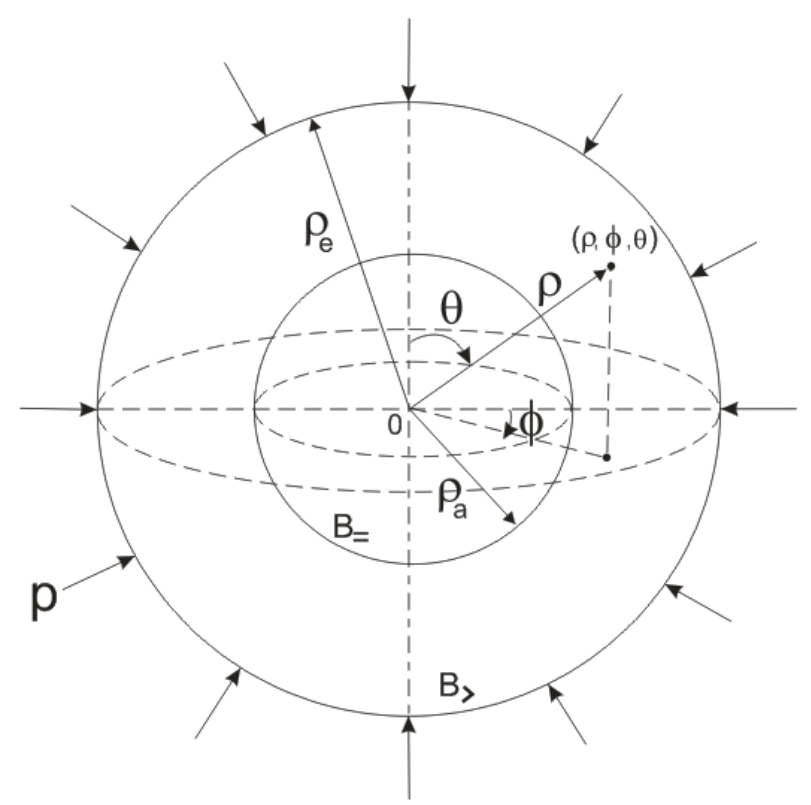

Figura 5.1. Problema da esfera com regiões onde a restrição da injetividade está ativa, ou, não-ativa.

Seja o gradiente da função mudança de configuração, definido por $\nabla f \equiv 1+\nabla u$, e sua 
inversa dados, respectivamente, por:

$$
\begin{aligned}
& \nabla f=\left(1+u^{\prime}\right) e_{\rho} \otimes e_{\rho}+(1+u / \rho) e_{\theta} \otimes e_{\theta}+(1+u / \rho) e_{\phi} \otimes e_{\phi} \\
& \nabla f^{-1}=\left(1+u^{\prime}\right)^{-1} e_{\rho} \otimes e_{\rho}+(1+u / \rho)^{-1} e_{\theta} \otimes e_{\theta}+(1+u / \rho)^{-1} e_{\phi} \otimes e_{\phi} .
\end{aligned}
$$

Seja $\Omega=\Omega_{=} \cup \Omega_{>} \subset \mathrm{R}^{3}$ a região ocupada pela esfera no seu estado natural dada por

$$
\begin{aligned}
& \Omega_{=}=\left\{\mathrm{x}=\rho \mathrm{e}_{\rho} \in \Omega: 0<\rho<\rho_{\mathrm{a}}\right\}, \\
& \Omega_{>}=\left\{\mathrm{x}=\rho \mathrm{e}_{\rho} \in \Omega: \rho_{\mathrm{a}}<\rho<\rho_{\mathrm{e}}\right\},
\end{aligned}
$$

onde $\rho_{\mathrm{a}} \in\left[0, \rho_{\mathrm{e}}\right]$ é um valor a ser determinado da solução do problema (Fig. 5.1).

As equações de Euler-Lagrange para as sub-regiões nas quais a restrição da injetividade local está ativa e não-ativa para o problema da esfera são dadas, respectivamente, por:

$$
\begin{aligned}
& \frac{d \sigma_{\rho \rho}}{d \rho}+\frac{2}{\rho}\left(\sigma_{\rho \rho}-\sigma_{\theta \theta}\right)=0 \quad \text { em } \quad\left(\rho_{\mathrm{a}}, \rho_{\mathrm{e}}\right), \\
& \frac{\mathrm{d}\left[\sigma_{\rho \rho}-\varepsilon \lambda\left(1+\mathrm{u}^{\prime}\right)^{-1}\right]}{\mathrm{d} \rho}+\frac{2}{\rho}\left\{\sigma_{\rho \rho}-\sigma_{\theta \theta}-\varepsilon \lambda\left[\left(1+\mathrm{u}^{\prime}\right)^{-1}-(1+\mathrm{u} / \rho)^{-1}\right]\right\}=0 \text { em }\left(0, \rho_{\mathrm{a}}\right) .
\end{aligned}
$$

No sub-intervalo $\left(0, \rho_{\mathrm{a}}\right)$, onde a restrição da injetividade está ativa, utiliza-se $\operatorname{det} \nabla f=\left(1+u^{\prime}\right)(1+u / \rho)^{2}=\varepsilon$ para obter a equação diferencial de primeira ordem

$$
\varepsilon=\frac{1}{3 \rho^{2}} \frac{d(\rho+u)^{3}}{d \rho} \text { em }\left(0, \rho_{\mathrm{a}}\right)
$$


Uma vez que a solução desta equação deve satisfazer a condição de compatibilidade $\mathrm{u}(0)=0$, obtém-se de (5.21) que

$$
u(\rho)=-\left(1-\varepsilon^{1 / 3}\right) \rho \text { em }\left(0, \rho_{a}\right) .
$$

Esta expressão fornece um campo de deslocamento que satisfaz a restrição da injetividade e elimina o fenômeno da auto-intersecção na região $\Omega_{=}$delimitada pelo raio $\rho_{\mathrm{a}}$.

Determina-se agora o campo de deslocamento em $\left(\rho_{\mathrm{a}}, \rho_{\mathrm{e}}\right)$. Substituindo-se as expressões (3.18) em (5.19), tem-se

$$
\frac{d^{2} u}{d \rho^{2}}+\frac{2}{\rho} \frac{d u}{d \rho}-2 \gamma \frac{u}{\rho^{2}}=0 \quad \text { em } \quad\left(\rho_{a}, \rho_{e}\right)
$$

onde $\gamma$ é dado por (3.21).

A solução geral de (5.23) é da forma (3.23), onde $\lambda^{ \pm}$e $\kappa$ são dados por (3.24) e as constantes $\alpha^{ \pm} \in \mathrm{R}$ são determinadas a partir da condição de contorno em $\rho_{\mathrm{e}}$ e condição de continuidade em $\rho=\rho_{\mathrm{a}}$. Para o problema da esfera, estas condições são dadas por

$$
\begin{aligned}
& u\left(\rho_{a}^{-}\right)=u\left(\rho_{a}^{+}\right), \\
& \sigma_{\rho \rho}\left(\rho_{a}^{-}\right)-\varepsilon \lambda\left(\rho_{a}^{-}\right)\left[1+u^{\prime}\left(\rho_{a}^{-}\right)\right]^{-1}=\sigma_{\rho \rho}\left(\rho_{a}^{+}\right), \\
& \sigma_{\rho \rho}\left(\rho_{e}\right)=-p,
\end{aligned}
$$

onde $\rho_{\mathrm{a}}^{ \pm} \equiv \lim _{\tau \rightarrow 0}\left(\rho_{\mathrm{a}} \pm \tau\right)$ para $\tau>0$ e $\sigma_{\rho \rho}$ é dado por (3.18a).

Substituindo-se (3.18) e (5.21) em (5.20), obtém-se a equação diferencial de primeira 
ordem que deve ser satisfeita na região $\Omega_{=}$,

$$
-\lambda^{\prime}(\rho)+\frac{\alpha}{\rho}=0
$$

onde $\alpha=2 \mathrm{C}_{11} \varepsilon^{-2 / 3}\left(-1-\gamma+\varepsilon^{1 / 3}-\gamma \varepsilon^{1 / 3}\right)$.

A solução geral de (5.27) é dada por

$$
\lambda(\rho)=\alpha \log \left(\frac{\rho}{\hat{\rho}}\right)
$$

para algum $\hat{\rho} \in \mathrm{R}$. Aguiar (2006) mostra que para $\lambda(\rho) \geq 0$ em $\Omega_{=}, \hat{\rho}=\rho_{\mathrm{a}}$.

Observa-se de (5.28) e (3.18) que $\lambda$ tem uma singularidade logarítmica na origem, que é uma singularidade mais fraca que a singularidade de tensões do problema sem restrição para o mesmo intervalo de $\kappa$, ou seja, para $0<\kappa<1$.

Utilizando-se as condições (5.24) e (5.25) juntamente com a expressão (5.22) determinam-se as constantes $\alpha^{ \pm}$, que são dadas por:

$$
\alpha^{ \pm}=\frac{(\mp 1-\kappa)}{2 \kappa}\left(1-\varepsilon^{1 / 3}\right) \rho_{\mathrm{a}}^{3(1 \mp \kappa) / 2}
$$

Para determinar $\rho_{\mathrm{a}}$ em (5.29), utiliza-se a condição de contorno (5.26). Substituindose (3.18a), (3.24) e (5.29) em (5.26), tem-se:

$$
\left(\lambda^{+}+2 \eta\right)\left(\frac{1+\kappa}{2 \kappa}\right) \zeta^{3(\kappa-1) / 2}-\left(\lambda^{-}+2 \eta\right)\left(\frac{1-\kappa}{2 \kappa}\right) \zeta^{-3(\kappa+1) / 2}-\frac{p}{C_{11}\left(1-\varepsilon^{1 / 3}\right)} \equiv g(\zeta)=0
$$


onde $\zeta \equiv \rho_{\mathrm{e}} / \rho_{\mathrm{a}}$ e $\eta \equiv \mathrm{C}_{12} / \mathrm{C}_{11}$. Aguiar (2006b) mostra que existe um único $\rho_{\mathrm{a}} \in\left[0, \rho_{\mathrm{e}}\right]$ que satisfaz a equação algébrica $\mathrm{g}\left(\rho_{\mathrm{e}} / \rho_{\mathrm{a}}\right)=0$, onde $\mathrm{g}$ é dado por (5.30), contanto que $\mathrm{p} \leq \mathrm{p}_{0} \equiv \mathrm{C}_{11}(1+2 \eta)\left(1-\varepsilon^{1 / 3}\right)$. Se $\mathrm{p}>\mathrm{p}_{0}$, então $\Omega_{>}=\varnothing$ e $\Omega_{=}=\Omega$

Utilizando-se (3.24), (3.25), (5.22) e (5.29), chega-se à expressão final para o campo de deslocamento $u=u(\rho)$, dada por

$$
u(\rho)=\frac{\left(1-\varepsilon^{1 / 3}\right) \rho_{a}}{2 \kappa}\left[-(1+\kappa)\left(\frac{\rho}{\rho_{a}}\right)^{-(1-3 \kappa) / 2}+(1+\kappa)\left(\frac{\rho}{\rho_{a}}\right)^{-(1+3 \kappa) / 2}\right] \text { em }\left(\rho_{a}, \rho_{e}\right),
$$

onde $\rho_{\mathrm{a}}$ é a solução única da equação algébrica (5.30).

Substituindo-se (5.31) em (5.15), chega-se à expressão:

$$
\operatorname{det} \nabla f(\rho)=\left[1+\chi_{1}\left(\frac{\rho}{\rho_{\mathrm{a}}}\right)\right]\left[1-\chi_{2}\left(\frac{\rho}{\rho_{\mathrm{a}}}\right)\right]^{2} \quad \text { em } \Omega_{>},
$$

onde $f(\rho)=\rho+u(\rho)$ e

$$
\begin{aligned}
& \chi_{1}(\xi) \equiv \frac{\left(1-\varepsilon^{1 / 3}\right)}{4 \kappa \xi^{3 / 2}}\left[(1+\kappa)(1-3 \kappa) \xi^{3 \mathrm{k} / 2}-(1-\kappa)(1+3 \kappa) \xi^{-3 \kappa / 2}\right], \\
& \chi_{2}(\xi) \equiv \frac{\left(1-\varepsilon^{1 / 3}\right)}{2 \kappa \xi^{3 / 2}}\left[(1+\kappa) \xi^{3 \mathrm{k} / 2}-(1-\kappa) \xi^{-3 \kappa / 2}\right], \quad \xi \equiv\left(\frac{\rho}{\rho_{\mathrm{a}}}\right) \geq 1 .
\end{aligned}
$$

Aguiar (2006b) mostra que $\operatorname{det} \nabla \mathrm{f} \geq \varepsilon$ em $\Omega_{>}$para $0<\kappa<1$ e que $\operatorname{det} \nabla f$ é contínuo e tem derivada contínua em $\rho=\rho_{\mathrm{a}}$. Além disso, det $\nabla \mathrm{f} \geq \varepsilon$ em $\Omega$, isto é, que a função 
mudança de configuração f é localmente injetiva em todo o domínio $\Omega$.

O autor mostra também que o campo de deformação da esfera descrito pelas equações (5.22) e (5.31) é globalmente injetivo, ou seja, $-u(\rho) / \rho<1$ para $\rho \in\left(0, \rho_{\mathrm{a}}\right)$. Para $\rho \in\left(\rho_{a}, \rho_{e}\right)$, observa-se de (5.31) e (5.34) que $-u(\rho) / \rho=\chi_{2}\left(\rho / \rho_{a}\right)$. Uma vez que $\chi_{2}(1)=1-\varepsilon^{1 / 3}<1, \quad \chi_{2}^{\prime}(\xi)<0$ para todo $\xi \in[1, \infty], \quad \chi_{2}^{\prime}(1)<0$, e $\lim _{\xi \rightarrow \infty} \chi_{2}(\xi)=\lim _{\xi \rightarrow \infty} \chi_{2}^{\prime}(\xi)=0$ encontra-se que $\chi_{2}(\xi)$ é uma função monotonicamente decrescente de $\xi$ que atinge seu valor máximo que é menor que 1 , em $\xi=1$ e tende assintoticamente a zero de cima quando $\xi \rightarrow \infty$. Portanto $-\mathrm{u}(\rho) / \rho<1$ para $\rho \in\left(\rho_{\mathrm{a}}, \rho_{\mathrm{e}}\right)$. 


\subsection{CONSIDERAÇÕES INICIAIS}

No Cap. 4 foram apresentados princípios que permitem obter diretamente a formulação variacional fraca de um problema quando a correspondente forma forte é desconhecida. A partir desta formulação fraca é possível obter soluções aproximadas para o problema empregando-se, por exemplo, o Método dos Elementos Finitos (MEF).

Neste capítulo, apresentam-se alguns conceitos do MEF, uma formulação que permite obter sequiências de soluções numéricas aproximadas para o problema da esfera sem restrição utilizando-se o Método dos Elementos Finitos de Galerkin Clássico (MEFGC) e duas formulações utilizando-se o Método dos Elementos Finitos de Galerkin Descontínuo (MEFGD).

A formulação variacional fraca para aplicação do MEFGC, descrita na Seção 6.5, é obtida a partir da integração ponderada da forma forte do problema apresentada no Cap. 3. Para a primeira formulação do MEFGD, descrita na Seção 6.6, utiliza-se o procedimento descrito por Castilho (2003). Nesta formulação aproximam-se simultaneamente os campos de deslocamento e de deformação infinitesimal. Considerando-se que o campo adicional de 
deformação na formulação variacional aumenta o número de graus de liberdade associados aos nós dos elementos finitos utilizados para discretizar o domínio e consequentemente o custo computacional, introduz-se uma formulação alternativa do MEFGD com o objetivo de reduzir o número de graus de liberdade. Nesta nova formulação, descrita na Seção 6.7, o campo de deformação infinitesimal não é obtido diretamente da inversão do sistema de equações lineares resultante, mas por pós-processamento, a partir dos valores de deslocamento obtidos.

\subsection{CARACTERÍSTICAS GERAIS DO MÉTODO DOS ELEMENTOS FINITOS DE GALERKIN CLÁSSICO}

Ciarlet (1979) define o Método dos Elementos Finitos de Galerkin Clássico, em sua forma mais simples, como um processo específico de construção dos subespaços $V_{h}$ chamados espaços de elementos finitos. A construção destes subespaços é caracterizada por três aspectos básicos:

1) A partição $T_{h}$ de um conjunto fechado $\bar{\Omega}$, ou seja, a subdivisão de $\bar{\Omega}$ em um número finito de subconjuntos I deve ser construída de forma que as seguintes propriedades sejam satisfeitas:

a) $\bar{\Omega}=\underset{\mathrm{I} \in \mathrm{T}_{\mathrm{h}}}{\cup} \mathrm{I}$, ou seja, o conjunto $\bar{\Omega}$ é descrito pela união finita dos subconjuntos $\mathrm{I} \in \mathrm{T}_{\mathrm{h}}$;

b) Para cada $I \in T_{h}$, o conjunto I é fechado e o interior I é não vazio;

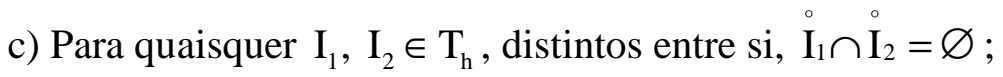

d) Para cada $I \in T_{h}$, o contorno $\partial \mathrm{I}$ é suficientemente suave; 
e) Uma interface ${ }^{2}$ qualquer de um subconjunto $I_{1}$ é também uma interface de um subconjunto $I_{2}$. Neste caso os subconjuntos $I_{1}$ e $I_{2}$ são adjacentes. Quando uma interface qualquer de um subconjunto não é interface de um subconjunto adjacente, ela é uma parte do contorno $\partial \Omega$ do conjunto $\Omega$.

2) Em geral, o espaço de funções reais $P_{I}$ associado a $I, I \in T_{h}$, contêm polinômios.

3) Existe pelo menos uma base canônica no espaço $V_{h}$, cujas correspondentes funções base têm suporte tão pequeno quanto possível, sendo que estas funções base podem ser facilmente descritas.

Um elemento finito $(\mathrm{I}, \Sigma, \mathrm{P})$ pode então ser definido como um subconjunto fechado I com interior não-vazio e contorno suficientemente suave, pertencente à partição $T_{h}$ do domínio $\bar{\Omega}$ com um conjunto finito $\Sigma$ de graus de liberdade $\alpha_{\mathrm{i}}, 1 \leq \mathrm{i} \leq \mathrm{N}$ e um espaço $\mathrm{P}_{\mathrm{I}}$ de funções reais associado.

\subsection{MÉTODO DOS ELEMENTOS FINITOS CONFORMES}

Seja V um espaço vetorial normado, $V_{h}$ um subespaço do espaço V, $\mathrm{a}[\cdot, \cdot]: \mathrm{V} \times \mathrm{V} \rightarrow \mathrm{R}$ uma forma bilinear contínua e $\mathrm{F}: \mathrm{V} \rightarrow \mathrm{R}$ uma forma linear contínua. Ciarlet (1979) reserva a terminologia Método dos Elementos Finitos Conforme para os Métodos de Elementos Finitos nos quais o espaço $V_{h}$ é um subespaço do espaço $V$, e as formas linear e bilinear do problema discreto são idênticas às formas linear e bilinear do problema original.

Neste sentido, o autor descreve três formas de violar a conformidade dos Métodos dos

\footnotetext{
${ }^{2}$ Por interface entende-se o dispositivo físico que faz a conexão entre dois elementos ou ainda a fronteira que define a forma de comunicação entre dois elementos finitos adjacentes, ou seja, a interface dos elementos finitos unidimensionais aqui estudados será o nó.
} 
Elementos finitos que ocorrem quando:

i) se tem problemas variacionais mais gerais ou problemas não-lineares;

ii) quando é necessário utilizar esquemas de quadratura para calcular as formas bilinear e linear do problema discreto, sendo que estas formas resultantes podem não ser idênticas a do problema original, ou não pertencer ao subespaço $\mathrm{V}_{\mathrm{h}}$;

iii) quando se utiliza elementos finitos que não são da classe das funções contínuas, $\mathrm{C}^{0}$, ou seja, do uso de funções admissíveis que não são contínuas entre elementos adjacentes.

\subsection{MÉTODO DOS ELEMENTOS FINITOS DE GALERKIN DESCONTÍNUO}

Basicamente, a diferença entre o Método de Galerkin Descontínuo e o Método de Galerkin Clássico reside na utilização de aproximações descontínuas $\mathrm{u}_{\mathrm{h}}$ entre elementos adjacentes e na construção da formulação variacional em que se aproxima além do campo de deslocamentos o campo de deformação diretamente. Além disto, na definição do Método de Galerkin Descontínuo surge o conceito de fluxo numérico cuja finalidade é impor de forma fraca a continuidade da solução na formulação variacional e introduzir ao elemento estudado as contribuições dos elementos adjacentes, uma vez que as aproximações descontínuas têm suporte apenas sobre o elemento estudado.

Desta forma, o Método de Galerkin Descontínuo pode ser definido com uma variação do Método de Galerkin Clássico em que a continuidade da solução entre elementos do domínio não é imposta através do espaço de interpolação, mas de forma fraca, através de fluxos numéricos sobre o contorno dos elementos, na formulação variacional (FORTI, 2005).

Com base nas descrições feitas na Seção 6.3, observa-se que o Método de Galerkin Descontínuo é um método não-conforme devido à utilização de funções admissíveis 
descontínuas entre elementos adjacentes.

\subsection{FORMULAÇÃO DISCRETA PARA O PROBLEMA DA ESFERA SEM RESTRIÇÃO UTILIZANDO-SE O MEFGC}

Seja uma esfera, homogênea, esfericamente ortotrópica, de raio $\rho_{\mathrm{e}}=1$, radialmente comprimida ao longo do seu contorno externo por uma força normal uniformemente distribuída por unidade de superfície $\mathrm{p}$. A formulação forte deste problema foi apresentada na Seção 3.7 e consiste em achar o deslocamento radial $\mathrm{u}:\left(0, \rho_{\mathrm{e}}\right) \rightarrow \mathrm{R}$ em um sistema de coordenadas esféricas $(\rho, \phi, \theta)$ com origem no centro da esfera $\Omega$, tal que a equação diferencial (3.20) juntamente com (3.21) e as condições de contorno (3.22) sejam satisfeitas.

A partir desta formulação forte constrói-se, nesta Seção, a formulação fraca e aplica-se o MEFGC para gerar seqüências de soluções aproximadas para o problema da esfera.

Seja $\mathrm{T}_{\mathrm{h}}=\left\{\rho_{\mathrm{i}}\right\}_{\mathrm{i}=1}^{\mathrm{n}+1}$ a partição de $\bar{\Omega}: 0=\rho_{1}<\rho_{2} \ldots<\rho_{\mathrm{n}+1}=1, \mathrm{I}_{\mathrm{i}}=\left(\rho_{\mathrm{i}}, \rho_{\mathrm{i}+1}\right), \bar{\Omega}=\bigcup_{\mathrm{i}=1}^{\mathrm{n}} \mathrm{I}_{\mathrm{i}}$.

Primeiramente, constrói-se a formulação variacional. Multiplicando-se ambos os lados da Eq. (3.20) por uma função $v \in V$ e lembrando de que ambos, u e v, são funções de $\rho$ somente, obtém-se

$$
4 \pi \int_{0}^{\rho_{\mathrm{e}}}\left(\frac{\mathrm{d}^{2} \mathrm{u}}{\mathrm{d} \rho^{2}}+\frac{2}{\rho} \frac{\mathrm{du}}{\mathrm{d} \rho}-2 \gamma \frac{\mathrm{u}}{\rho^{2}}\right) \mathrm{v} \rho^{2} \mathrm{~d} \rho=0, \quad \forall \mathrm{v} \in \mathrm{V},
$$

onde $\mathrm{V}=\left\{\mathrm{v} \in \mathrm{C}^{0}\left(0, \rho_{\mathrm{e}}\right): \mathrm{v}(0)=0\right\}, \gamma$ é dado por (3.21) e as condições de contorno são dadas por (3.22).

Integrando-se a Eq. (6.1) por partes e utilizando-se as condições de contorno (3.22), 
chega-se à formulação variacional fraca do problema da esfera, que consiste em achar $u \in U$, tal que

$$
\int_{0}^{\rho_{\mathrm{e}}} \frac{\mathrm{du}}{\mathrm{d} \rho} \frac{\mathrm{dv}}{\mathrm{d} \rho} \rho^{2} \mathrm{~d} \rho+\int_{0}^{\rho_{\mathrm{e}}} 2 \gamma u v d \rho+2 \frac{\mathrm{C}_{12}}{\mathrm{C}_{11}} \mathrm{u}\left(\rho_{\mathrm{e}}\right) \mathrm{v}\left(\rho_{\mathrm{e}}\right) \rho_{\mathrm{e}}+\frac{\mathrm{p}}{\mathrm{C}_{11}} \rho_{\mathrm{e}}^{2} \mathrm{v}\left(\rho_{\mathrm{e}}\right)=0, \quad \forall \mathrm{v} \in \mathrm{V} .
$$

Seja agora $\mathrm{V}_{\mathrm{h}}$ o conjunto das funções $\mathrm{v}_{\mathrm{h}}$, tal que

$$
\mathrm{V}_{\mathrm{h}}=\left\{\mathrm{v}_{\mathrm{h}} \in \mathrm{C}^{0}\left(0, \rho_{\mathrm{e}}\right): \mathrm{v}_{\mathrm{h}}(0)=0\right\}
$$

onde $\mathrm{v}_{\mathrm{h}}$ é linear em cada sub-intervalo $\mathrm{I}_{\mathrm{i}}, \mathrm{v}_{\mathrm{h}}$ é contínua em $\Omega=(0,1)$ e portanto, $\mathrm{V}_{\mathrm{h}} \subset \mathrm{V}$. Seja também $\mathrm{U}_{\mathrm{h}}=\mathrm{V}_{\mathrm{h}}$, de modo que $\mathrm{U}_{\mathrm{h}} \subset \mathrm{U}$.

O problema variacional discreto correspondente a (6.2) consiste em achar $\mathrm{u}_{\mathrm{h}} \in \mathrm{U}_{\mathrm{h}}$, tal que:

$$
\int_{0}^{\rho_{\mathrm{e}}} \frac{d u_{\mathrm{h}}}{\mathrm{d} \rho} \frac{\mathrm{d} \mathrm{v}_{\mathrm{h}}}{\mathrm{d} \rho} \rho^{2} \mathrm{~d} \rho+\int_{0}^{\rho_{\mathrm{e}}} 2 \gamma \mathrm{u}_{\mathrm{h}} \mathrm{v}_{\mathrm{h}} \mathrm{d} \rho+2 \frac{\mathrm{C}_{12}}{\mathrm{C}_{11}} \mathrm{u}_{\mathrm{h}}\left(\rho_{\mathrm{e}}\right) \mathrm{v}_{\mathrm{h}}\left(\rho_{\mathrm{e}}\right) \rho_{\mathrm{e}}+\frac{\mathrm{p}}{\mathrm{C}_{11}} \rho_{\mathrm{e}}^{2} \mathrm{v}_{\mathrm{h}}\left(\rho_{\mathrm{e}}\right)=0, \quad \forall \mathrm{v}_{\mathrm{h}} \in \mathrm{V}_{\mathrm{h}},
$$

onde $\gamma$ é dado por (3.21).

Uma função $\mathrm{v}_{\mathrm{h}} \in \mathrm{V}_{\mathrm{h}}$ pode ser escrita na forma

$$
\mathrm{v}_{\mathrm{h}}(\rho)=\sum_{\mathrm{i}=2}^{\mathrm{n}+1} \mathrm{v}_{\mathrm{i}} \phi_{\mathrm{i}}(\rho), \quad \rho \in(0,1),
$$

onde considera-se que o conjunto $\left\{\phi_{i}:(0,1) \rightarrow R, i=1, \ldots, n+1\right\}$ é uma base para o espaço 
finito dimensional $V_{h}$ e que os elementos $\phi_{i}$ desta base satisfazem as condições de normalidade dadas pela expressão (6.6) e ilustrada na Fig. 6.1.

$$
\phi_{\mathrm{i}}\left(\rho_{\mathrm{j}}\right)=\phi_{\mathrm{j}}\left(\rho_{\mathrm{i}}\right)=\left\{\begin{array}{lll}
1 & \text { se } & \mathrm{i}=\mathrm{j} \\
0 & \text { se } & \mathrm{i} \neq \mathrm{j}
\end{array} \quad \mathrm{i}, \mathrm{j}=1, \ldots \mathrm{n}+1 .\right.
$$

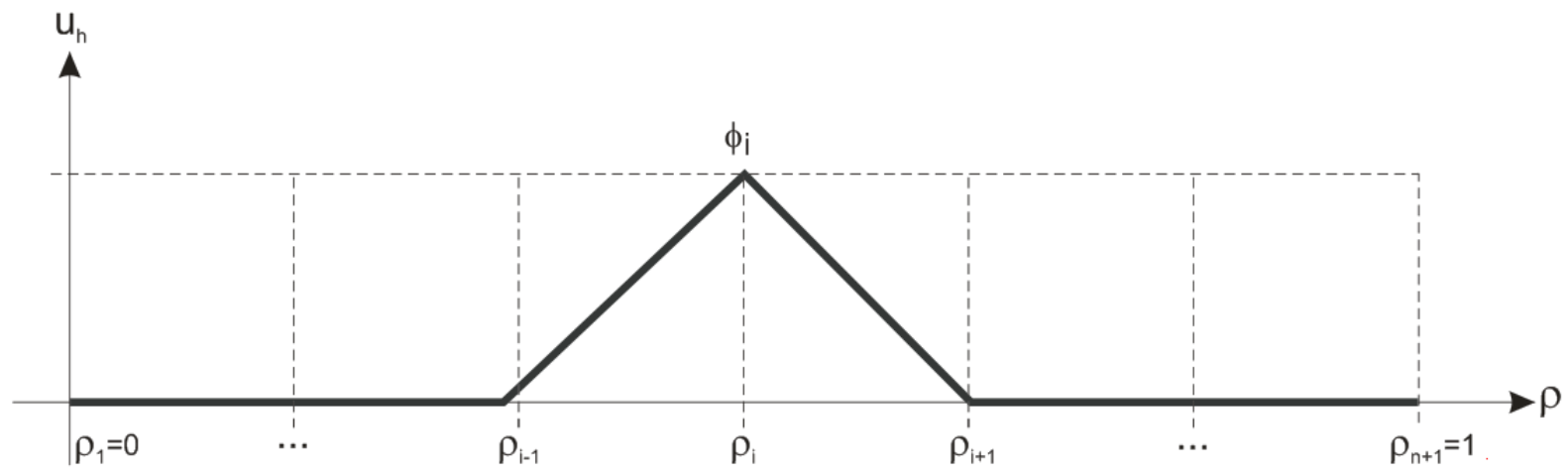

Figura 6.1. Função tenda.

Observa-se de (6.5) juntamente com (6.6) que os coeficientes $v_{i}, i=1, \ldots, n+1$ satisfazem a relação $v_{i}=v_{h}\left(\rho_{i}\right)$.

A expressão (6.5) permite escrever $\mathrm{V}_{\mathrm{h}} \in \mathrm{V}_{\mathrm{h}}$ de forma única como uma combinação linear das funções base $\phi_{\mathrm{i}}$.

As funções base adotadas são lineares por partes e contínuas. Para os elementos intermediários do intervalo, ou seja, para $\mathrm{i}=2, \ldots, \mathrm{n}$, as funções base são dadas por

$$
\phi_{i}(\rho)=\left\{\begin{array}{cc}
\frac{\rho-\rho_{i-1}}{\rho_{i}-\rho_{i-1}}, & \rho_{i-1} \leq \rho \leq \rho_{i}, \\
\frac{\rho_{i+1}-\rho}{\rho_{i+1}-\rho_{i}}, & \rho_{i} \leq \rho \leq \rho_{i+1}, \\
0, & \text { de outra forma. }
\end{array} .\right.
$$


Para os elementos próximos às extremidades do intervalo, tem-se que

$\phi_{1}(\rho)=\left\{\begin{array}{cc}\frac{\rho_{2}-\rho}{\rho_{2}-\rho_{i}}, & 0=\rho_{1} \leq \rho \leq \rho_{2}, \\ 0, & \text { de outra forma. }\end{array}, \quad \phi_{n+1}(\rho)=\left\{\begin{array}{cc}\frac{\rho-\rho_{n}}{\rho_{n+1}-\rho_{n}}, & \rho_{n} \leq \rho \leq \rho_{n+1}=1, \\ 0, & \text { de outra forma. }\end{array}\right.\right.$

Substituindo-se (6.5) em (6.4), levando-se em consideração as expressões (6.7) e (6.8) e uma vez que $\mathrm{v}_{\mathrm{h}}(0)=0$, tem-se que

$$
\sum_{i=2}^{n+1} v_{i}\left[\int_{0}^{\rho_{e}} \frac{d u_{h}}{d \rho} \phi_{i}^{\prime}(\rho) \rho^{2} d \rho+\int_{0}^{\rho_{e}} 2 \gamma u_{h} \phi_{i}(\rho) d \rho+2 \frac{C_{12}}{C_{11}} u_{h}\left(\rho_{e}\right) \delta_{i(n+1)} \rho_{e}+\frac{p}{C_{11}} \rho_{e}^{2} \delta_{i(n+1)}\right]=0
$$

onde $\delta_{\mathrm{ij}}$ é o Delta de Kronecker. A expressão (6.9) é válida $\forall \mathrm{v}_{\mathrm{i}} \in \mathrm{R}$.

Como $\mathrm{v}_{\mathrm{i}}$ é arbitrário, tem-se que

$$
\int_{0}^{\rho_{e}} \frac{d u_{h}}{d \rho} \phi_{i}^{\prime}(\rho) \rho^{2} d \rho+\int_{0}^{\rho_{e}} 2 \gamma u_{h} \phi_{i}(\rho) d \rho+2 \frac{C_{12}}{C_{11}} u_{h}\left(\rho_{e}\right) \delta_{i(n+1)} \rho_{e}+\frac{p}{C_{11}} \rho_{e}^{2} \delta_{i(n+1)}=0
$$

onde $\mathrm{i}=2, \ldots, \mathrm{n}+1$.

Substituindo-se $u_{h}=\sum_{j=1}^{n+1} u_{j} \phi_{j}(\rho)$ em (6.10) e uma vez que $u_{h}(0)=0$, tem-se que

$$
\sum_{j=2}^{n+1} u_{j}\left[\int_{0}^{\rho_{e}} \phi_{j}^{\prime}(\rho) \phi_{i}^{\prime}(\rho) \rho^{2} d \rho+\int_{0}^{\rho_{e}} 2 \gamma \phi_{j}(\rho) \phi_{i}(\rho) d \rho+2 \frac{C_{12}}{C_{11}} \phi_{j}\left(\rho_{e}\right) \delta_{i(n+1)} \rho_{e}\right]+\frac{p}{C_{11}} \rho_{e}^{2} \delta_{i(n+1)}=0,
$$

onde $i=2, \ldots, n+1$. 
Substituindo-se (6.7)-(6.8) em (6.11) e considerando-se uma discretização com n elementos tem-se um sistema com $n$ equações e $n$ incógnitas $u_{j}$.

\subsection{FORMULAÇÃO DISCRETA PARA O PROBLEMA DA ESFERA SEM RESTRIÇÃO UTILIZANDO-SE O MEFGD}

Considera-se novamente o problema descrito pelas expressões (3.20)-(3.22).

Introduzindo-se a variável $\mathrm{q}=\frac{\mathrm{du}}{\mathrm{d} \rho}$, a equação (3.20) pode ser escrita como um sistema de equações de primeira ordem da forma

$$
\begin{array}{ll}
\frac{d u}{d \rho}=q & \text { em }(0,1), \\
\frac{d q}{d \rho}+2 q \frac{1}{\rho}-2 \gamma \frac{u}{\rho^{2}}=0 & \text { em }(0,1),
\end{array}
$$

onde $\gamma$ é dado por (3.21) e as condições de contorno essencial e natural são dadas por (3.22).

Seja $U=V=\prod_{I_{i}} H^{1}\left(I_{i}\right)$, onde $H^{1}\left(I_{i}\right)$ é o espaço das funções quadrado integráveis com derivadas também quadrado integráveis em $I_{i}$, e seja novamente $T_{h}=\left\{\rho_{i}\right\}_{i=1}^{n+1}$ a partição de $\bar{\Omega}, 0=\rho_{1}<\rho_{2} \ldots<\rho_{n+1}=1, I_{i}=\left(\rho_{i}, \rho_{i+1}\right), \bar{\Omega}=\bigcup_{i=1}^{n} I_{i}$

Sejam (t,v) em VxU funções diferenciáveis por partes com suporte em $I_{i}$. Multiplicando-se as equações (6.12) e (6.13), em cada elemento $I_{i}$, pelas funções teste t e v, respectivamente, e integrando-se sobre $I_{i}$, obtêm-se as equações 


$$
\begin{aligned}
& \int_{\rho_{i}}^{\rho_{i+1}} \frac{d u}{d \rho} t \rho^{2} d \rho=\int_{\rho_{i}}^{\rho_{i+1}} q t \rho^{2} d \rho, \\
& \int_{\rho_{i}}^{\rho_{i+i}}\left(\frac{d q}{d \rho} v+2 q \frac{1}{\rho} v-2 \gamma \frac{u_{\rho}}{\rho^{2}} v\right) \rho^{2} d \rho=0 .
\end{aligned}
$$

Integrando-se as equações (6.14) e (6.15) por partes, chega-se às equações:

$$
\begin{aligned}
& \left(u \operatorname{ut} \rho^{2}\right)\left(\rho_{i+1}^{-}\right)-\left(u \rho^{2}\right)\left(\rho_{i}^{+}\right)-\int_{\rho_{i}}^{\rho_{i+1}}\left(u \frac{d t}{d \rho} \rho^{2}+2 u t \rho\right) d \rho=\int_{\rho_{i}}^{\rho_{i+1}} q t \rho^{2} d \rho, \\
& \left(q v \rho^{2}\right)\left(\rho_{i+1}^{-}\right)-\left(q v \rho^{2}\right)\left(\rho_{i}^{+}\right)=\int_{\rho_{i}}^{\rho_{i+1}}\left(q \frac{d v}{d \rho} \rho^{2}+2 \gamma u v\right) d \rho .
\end{aligned}
$$

As equações (6.16) e (6.17) são bem definidas para quaisquer funções (u,q) e (t,v) em VxU.

Aproxima-se a solução exata $(\mathrm{u}, \mathrm{q})$ com funções $\left(\mathrm{u}_{\mathrm{h}}, \mathrm{q}_{\mathrm{h}}\right)$ no espaço de elementos finitos $U_{h} x V_{h} \subset U x V$ onde $U_{h}=V_{h}=\prod_{I_{i}} P_{p}\left(I_{i}\right)$. O espaço de elementos finitos local $P_{p}\left(I_{i}\right)$ é o conjunto dos polinômios de grau menor ou igual a $\mathrm{p}$ definidos sobre $\mathrm{I}_{\mathrm{i}}$.

Desta forma, a formulação variacional fraca do problema (3.20)-(3.22) consiste em $\operatorname{achar}\left(\mathrm{u}_{\mathrm{h}}, \mathrm{q}_{\mathrm{h}}\right) \in \mathrm{U}_{\mathrm{h}} \mathrm{x} \mathrm{V}_{\mathrm{h}}$ para qualquer $(\mathrm{t}, \mathrm{v}) \in \mathrm{U}_{\mathrm{h}} \mathrm{xV}_{\mathrm{h}}$ que satisfaça

$$
\begin{aligned}
& \int_{\rho_{i}}^{\rho_{i+1}} q_{h} t \rho^{2} d \rho=\hat{u}_{h} t \rho^{2}\left(\rho_{i+1}^{-}\right)-\hat{u}_{h} t \rho^{2}\left(\rho_{i}^{+}\right)-\int_{\rho_{i}}^{\rho_{i+1}}\left(u_{h} \frac{d t}{d \rho} \rho^{2}+2 u_{h} t \rho\right) d \rho, \\
& \int_{\rho_{i}}^{\rho_{i+1}}\left(q_{h} \frac{d v}{d \rho} \rho^{2}+2 \gamma u_{h} v\right) d \rho=\hat{q}_{h} v \rho^{2}\left(\rho_{i+1}^{-}\right)-\hat{q}_{h} v \rho^{2}\left(\rho_{i}^{+}\right),
\end{aligned}
$$

onde $\hat{\mathrm{u}}_{\mathrm{h}}$ e $\hat{\mathrm{q}}_{\mathrm{h}}$ são chamados fluxos numéricos e podem ser entendidos como aproximações para os traços das funções $\mathrm{u}$ e q , respectivamente. A escolha dos fluxos numéricos 
determina as propriedades numéricas do método, tais como estabilidade, ordem de convergência, simetria, etc., (CASTILLO, 2003).

Para introduzir a definição de fluxo numérico, define-se, primeiramente a média $\{\{\cdot\}\}$ e salto $\langle\cdot\rangle$ para $\mathrm{v} \in \mathrm{V}=\mathrm{U}$, como

$$
\begin{aligned}
& \{\{\mathrm{v}\}\}=\frac{1}{2}\left(\mathrm{v}\left(\rho_{\mathrm{i}}^{-}\right)+\mathrm{v}\left(\rho_{\mathrm{i}}^{+}\right)\right), \\
& \langle\mathrm{v}\rangle=\mathrm{v}\left(\rho_{\mathrm{i}}^{-}\right)-\mathrm{v}\left(\rho_{\mathrm{i}}^{+}\right)
\end{aligned}
$$

Assim, definem-se os fluxos numéricos $\hat{\mathrm{u}}_{\mathrm{h}}$ e $\hat{\mathrm{q}}_{\mathrm{h}}$ como

$$
\begin{aligned}
& \hat{\mathrm{u}}_{\mathrm{h}}=\left\{\left\{\mathrm{u}_{\mathrm{h}}\right\}\right\}, \\
& \hat{\mathrm{q}}_{\mathrm{h}}=\left\{\left\{\mathrm{u}_{\mathrm{h}}^{\prime}\right\}\right\}-\left\langle\mathrm{u}_{\mathrm{h}}\right\rangle \eta_{\mathrm{i}} / \mathrm{h}_{\mathrm{i}},
\end{aligned}
$$

onde $\eta_{\mathrm{i}}=1$ e $\mathrm{h}_{\mathrm{i}}=\rho_{\mathrm{i}+1}-\rho_{\mathrm{i}}$.

Os fluxos numéricos $\hat{\mathrm{u}}_{\mathrm{h}}$ e $\hat{\mathrm{q}}_{\mathrm{h}}$ utilizados neste trabalho para os nós intermediários do intervalo $\left(0, \rho_{\mathrm{e}}\right)$, são dados, respectivamente, por:

$$
\begin{aligned}
& \hat{\mathrm{u}}_{\mathrm{h}}\left(\rho_{\mathrm{i}}\right)=\frac{\mathrm{u}_{\mathrm{h}}\left(\rho_{\mathrm{i}}^{-}\right)+\mathrm{u}_{\mathrm{h}}\left(\rho_{\mathrm{i}}^{+}\right)}{2}, \\
& \hat{\mathrm{q}}_{\mathrm{h}}\left(\rho_{\mathrm{i}}\right)=\frac{\mathrm{q}_{\mathrm{h}}\left(\rho_{\mathrm{i}}^{-}\right)+\mathrm{q}_{\mathrm{h}}\left(\rho_{\mathrm{i}}^{+}\right)}{2}-\frac{\mathrm{u}_{\mathrm{h}}\left(\rho_{\mathrm{i}}^{-}\right)-\mathrm{u}_{\mathrm{h}}\left(\rho_{\mathrm{i}}^{+}\right)}{\mathrm{h}_{\mathrm{i}}} .
\end{aligned}
$$

onde $\rho_{i}, i=2, \ldots, n$. 
O fluxo $\hat{\mathrm{u}}_{\mathrm{h}}(0)$ é dado pela condição de contorno de Dirichlet, ou seja, $\hat{\mathrm{u}}_{\mathrm{h}}(0)=\mathrm{u}_{0}$. $\mathrm{O}$ fluxo $\hat{\mathrm{q}}_{\mathrm{h}}(0)$ pode ser obtido substituindo-se na definição (6.23) o salto $\left\langle\mathrm{u}_{\mathrm{h}}\right\rangle$, por $-\mathrm{u}_{\mathrm{h}}\left(0^{+}\right)$e a média $\left\{\left\{\mathrm{u}_{\mathrm{h}}^{\prime}\right\}\right\}$ por $\mathrm{u}_{\mathrm{h}}^{\prime}\left(0^{+}\right)$. Utilizando-se procedimento similar em $\rho=\rho_{\mathrm{e}}$, obtêm-se as expressões finais dos fluxos nas extremidades do intervalo $\left(0, \rho_{\mathrm{e}}\right)$, que são dadas por

$$
\begin{array}{ll}
\hat{\mathrm{u}}_{\mathrm{h}}(0)=\mathrm{u}_{0}=0, \quad \hat{\mathrm{q}}_{\mathrm{h}}(0)=\mathrm{q}_{\mathrm{h}}\left(0^{+}\right)+\frac{\mathrm{u}_{\mathrm{h}}\left(0^{+}\right)}{\mathrm{h}_{1}}, \\
\hat{\mathrm{u}}_{\mathrm{h}}\left(\rho_{\mathrm{e}}\right)=\mathrm{u}_{\mathrm{h}}\left(\rho_{\mathrm{e}}^{-}\right), \quad \hat{\mathrm{q}}_{\mathrm{h}}\left(\rho_{\mathrm{e}}\right)=-\frac{\mathrm{p}}{\mathrm{C}_{11}}-2 \frac{\mathrm{C}_{12}}{\mathrm{C}_{11}} \frac{\mathrm{u}_{\mathrm{h}}\left(\rho_{\mathrm{e}}^{-}\right)}{\rho_{\mathrm{e}}} .
\end{array}
$$

As soluções $\left(\mathrm{u}_{\mathrm{h}}, \mathrm{q}_{\mathrm{h}}\right)$ foram aproximadas por funções lineares por partes e descontínuas nas interfaces entre elementos. Sendo assim, as soluções aproximadas $\left(\mathrm{u}_{\mathrm{h}}, \mathrm{q}_{\mathrm{h}}\right)$ são dadas, respectivamente, por

$$
\begin{aligned}
& u_{h}(\rho)=u_{h}\left(\rho_{i}^{+}\right)-\frac{u_{h}\left(\rho_{i+1}^{-}\right)-u_{h}\left(\rho_{i}^{+}\right)}{\rho_{i+1}-\rho_{i}} \rho_{i}+\frac{u_{h}\left(\rho_{i+1}^{-}\right)-u_{h}\left(\rho_{i}^{+}\right)}{\rho_{i+1}-\rho_{i}} \rho, \\
& q_{h}(\rho)=q_{h}\left(\rho_{i}^{+}\right)-\frac{q_{h}\left(\rho_{i+1}^{-}\right)-q_{h}\left(\rho_{i}^{+}\right)}{\rho_{i+1}-\rho_{i}} \rho_{i}+\frac{q_{h}\left(\rho_{i+1}^{-}\right)-q_{h}\left(\rho_{i}^{+}\right)}{\rho_{i+1}-\rho_{i}} \rho .
\end{aligned}
$$

Sendo $(\mathrm{t}, \mathrm{v})$ polinômios de primeiro grau pode-se escrever $\mathrm{t}=\mathrm{a}+\mathrm{b} \rho \mathrm{e} \mathrm{v}=\mathrm{c}+\mathrm{d} \rho$, onde a, b, c e d são coeficientes arbitrários.

Constroem-se então três sistemas de equações lineares, sendo um para os elementos intermediários do intervalo $\left(0, \rho_{\mathrm{e}}\right), \mathrm{I}_{\mathrm{i}}, \mathrm{i}=2, \ldots, \mathrm{n}-1$, um para o elemento $\mathrm{I}_{1}$ e um para $\mathrm{o}$ elemento $I_{n}$. 
Para obter o sistema de equações dos elementos intermediários substitui-se $t=a+b \rho$, $\mathrm{v}=\mathrm{c}+\mathrm{d} \rho,(6.24),(6.25),(6.28)$ e (6.29), em (6.18) e (6.19). Por simplicidade, mostram-se abaixo, as expressões (6.18) e (6.19) com a substituição apenas dos valores de t e v, respectivamente

$$
\begin{aligned}
& \int_{\rho_{\mathrm{i}}}^{\rho_{i+1}} \mathrm{q}_{\mathrm{h}}(\mathrm{a}+\mathrm{b} \rho) \rho^{2} \mathrm{~d} \rho=\hat{\mathrm{u}}_{\mathrm{h}}(\mathrm{a}+\mathrm{b} \rho) \rho^{2}\left(\rho_{\mathrm{i}+1}^{-}\right)-\hat{\mathrm{u}}_{\mathrm{h}}(\mathrm{a}+\mathrm{b} \rho) \rho^{2}\left(\rho_{\mathrm{i}}^{+}\right)- \\
& \int_{\rho_{\mathrm{i}}}^{\rho_{i+1}}\left(\mathrm{u}_{\mathrm{h}} \mathrm{b} \rho^{2}+2 \mathrm{u}_{\mathrm{h}}(\mathrm{a}+\mathrm{b} \rho) \rho\right) \mathrm{d} \rho \\
& \int_{\rho_{\mathrm{i}}}^{\rho_{\mathrm{i}+1}}\left(\mathrm{q}_{\mathrm{h}} \mathrm{d} \rho^{2}+2 \gamma \mathrm{u}_{\mathrm{h}}(\mathrm{c}+\mathrm{d} \rho)\right) \mathrm{d} \rho=\hat{\mathrm{q}}_{\mathrm{h}}(\mathrm{c}+\mathrm{d} \rho)^{2}\left(\rho_{\mathrm{i}+1}^{-}\right)-\hat{\mathrm{q}}_{\mathrm{h}}(\mathrm{c}+\mathrm{d} \rho) \rho^{2}\left(\rho_{\mathrm{i}}^{+}\right) .
\end{aligned}
$$

Fazendo-se todas as operações necessárias em (6.30) e agrupando-se os termos que possuem os coeficientes a e b em comum e procedendo-se de maneira similar em (6.31) em relação aos termos associados aos coeficientes $\mathrm{c}$ e d, chega-se a um sistema esquematicamente representado por

$$
\begin{aligned}
& \mathrm{a}(\cdot)_{1}+\mathrm{b}(\cdot)_{2}=0, \\
& \mathrm{c}(\cdot)_{3}+\mathrm{d}(\cdot)_{4}=0 .
\end{aligned}
$$

Como os coeficientes a, b, c e d são arbitrários, implica que os termos (.) em (6.32) são nulos,

$$
(\cdot)_{1}=0, \quad(\cdot)_{2}=0, \quad(\cdot)_{3}=0, \quad(\cdot)_{4}=0,
$$

ou seja, obtêm-se um sistema de quatro equações lineares em cada elemento $I_{i}=\left(\rho_{i}^{+}, \rho_{i+1}^{-}\right)$, 
$\mathrm{i}=2, \ldots, \mathrm{n}-1$ para oito incógnitas, a saber
i) $\mathrm{u}_{\mathrm{h}}\left(\rho_{\mathrm{i}}^{-}\right), \mathrm{q}_{\mathrm{h}}\left(\rho_{\mathrm{i}}^{-}\right)$pela esquerda do nó $\rho_{\mathrm{i}}$;
ii) $\mathrm{u}_{\mathrm{h}}\left(\rho_{\mathrm{i}}^{+}\right), \mathrm{q}_{\mathrm{h}}\left(\rho_{\mathrm{i}}^{+}\right)$pela direita do nó $\rho_{\mathrm{i}}$;
iii) $u_{h}\left(\rho_{i+1}^{-}\right), q_{h}\left(\rho_{i+1}^{-}\right)$pela esquerda do nó $\rho_{i+1}$;
iv) $u_{h}\left(\rho_{i+1}^{+}\right), q_{h}\left(\rho_{i+1}^{+}\right)$pela direita do nó $\rho_{i+1}$.

Observa-se que sobre cada nó do interior do intervalo $\left(0, \rho_{\mathrm{e}}\right)$ associam-se dois graus de liberdade em deslocamento $\mathrm{u}_{\mathrm{h}}$, que correspondem aos valores do deslocamento pela direita e pela esquerda do nó (Fig. 6.2). Similarmente, associam-se dois graus de liberdade para a derivada do deslocamento $\mathrm{q}_{\mathrm{h}}$, resultando em quatro graus de liberdade por nó (Fig. $6.2)$.

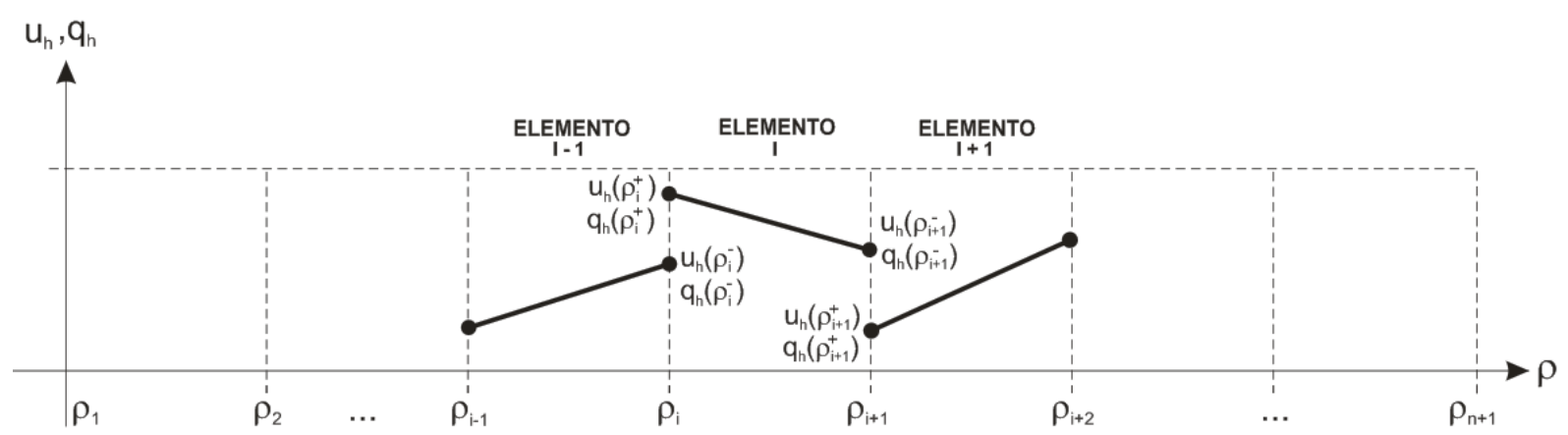

Figura 6.2. Graus de liberdade dos elementos intermediários na formulação original do MEFGD.

Para obter o sistema de equações do elemento $I_{1}$ substitui-se $t=a+b \rho, v=c+d \rho$, (6.24), (6.25) e (6.26), em (6.18) e (6.19). Para o elemento $I_{n}$ substitui-se $t=a+b \rho$, $v=c+d \rho,(6.24),(6.25)$ e (6.27), em (6.18) e (6.19). Procedendo-se de maneira similar ao descrito para os elementos intermediários, obtém-se um sistema de quatro equações e seis incógnitas para o primeiro e para o último elemento. 
Para o primeiro elemento do intervalo, os graus de liberdade estão associados aos nós conforme descrito abaixo:

i) $\mathrm{u}_{\mathrm{h}}\left(\rho_{1}^{+}\right), \mathrm{q}_{\mathrm{h}}\left(\rho_{1}^{+}\right)$pela direita do nó $\rho_{1}$;

ii) $u_{h}\left(\rho_{2}^{-}\right), q_{h}\left(\rho_{2}^{-}\right)$pela esquerda do nó $\rho_{2}$;

iii) $\mathrm{u}_{\mathrm{h}}\left(\rho_{2}^{+}\right), \mathrm{q}_{\mathrm{h}}\left(\rho_{2}^{+}\right)$pela direita do nó $\rho_{2}$.

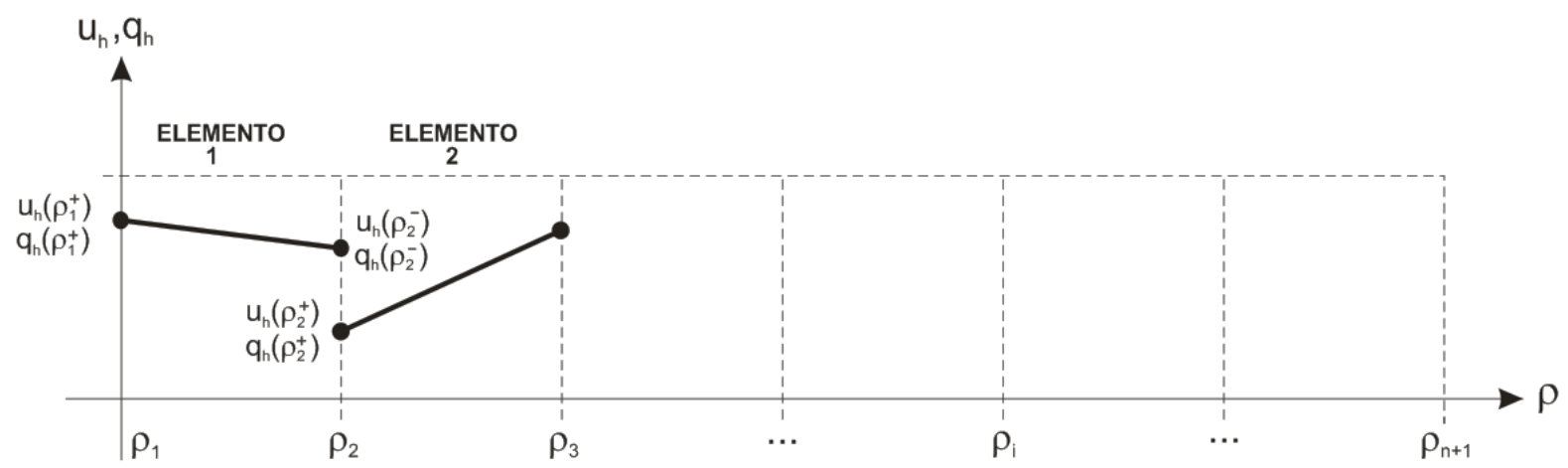

Figura 6.3. Graus de liberdade do elemento 1 na formulação original do MEFGD.

Aos nós do último elemento do intervalo associam-se os graus de liberdade conforme descrito abaixo:

i) $u_{h}\left(\rho_{n}^{-}\right), q_{h}\left(\rho_{n}^{-}\right)$pela esquerda do nó $\rho_{n}$;

ii) $u_{h}\left(\rho_{n}^{+}\right), q_{h}\left(\rho_{n}^{+}\right)$pela direita do nó $\rho_{n}$;

iii) $u_{h}\left(\rho_{n+1}^{-}\right), q_{h}\left(\rho_{n+1}^{-}\right)$pela esquerda do nó $\rho_{n+1}$.

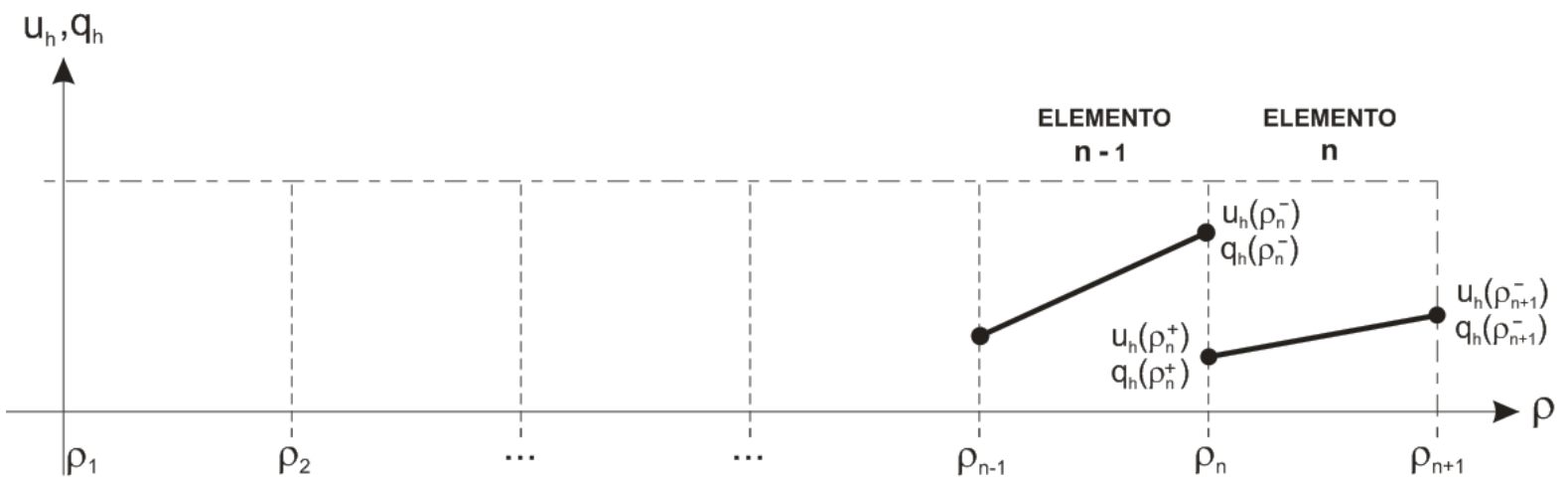

Figura 6.4. Graus de liberdade do elemento n na formulação original do MEFGD. 
Observa-se das Figs. 6.3 e 6.4 que sobre as extremidades do intervalo associam-se dois graus de liberdade que correspondem aos valores do deslocamento e de sua derivada pelo lado interior do intervalo e sobre o nó pertencente ao interior do domínio associam-se os 4 graus de liberdade igualmente aos nós dos elementos intermediários.

Sendo n o número de elementos finitos utilizados para discretizar o domínio, tem-se um sistema de $4 \mathrm{n}$ equações com $4 \mathrm{n}$ incógnitas a serem determinadas. A solução deste sistema são os valores de $\mathrm{u}_{\mathrm{h}}$ e $\mathrm{q}_{\mathrm{h}}$ pela direita e pela esquerda dos nós intermediários e os valores de $\mathrm{u}_{\mathrm{h}}$ e $\mathrm{q}_{\mathrm{h}}$ sobre os nós das extremidades do intervalo.

Uma vez que as aproximações $u_{h}$ e $q_{h}$ obtidas são descontínuas sobre os nós, utilizam-se as definições $\hat{\mathrm{u}}=\mathrm{u}$ e $\hat{\mathrm{q}}=\mathrm{u}^{\prime}$ (CASTILLO, 2003) juntamente com as definições (6.24)-(6.27) para se obter as soluções aproximadas $u_{h}$ e $q_{h}$ sobre os nós.

Por fim, observa-se que com a introdução da variável q esta formulação do MEFGD pode ser classificada como mista, pois aproxima diretamente os campos de deslocamento e deformação.

\subsection{FORMULAÇÃO ALTERNATIVA DO MEFGD}

Considerando-se o elevado número de graus de liberdade associados aos nós dos elementos finitos resultantes da aplicação da formulação original do MEFGD apresentada na Seção 6.6, introduz-se uma formulação variacional alternativa para aplicação do método em que apenas os valores de $u_{h}$ são obtidos diretamente da inversão do sistema de equações resultante, sendo os valores de $\mathrm{q}_{\mathrm{h}}$ obtidos por pós-processamento a partir dos valores de $\mathrm{u}_{\mathrm{h}}$.

Similarmente ao realizado na Seção 6.6 introduz-se a equação diferencial $\frac{d u}{d \rho}=q$. Aqui, no entanto, esta equação não é integrada por partes e o problema (3.20)-(3.22) passa a 
ser descrito pelo sistema de equações de primeira ordem dado por

$$
\begin{aligned}
& \frac{d u}{d \rho}=q \\
& \int_{0}^{\rho_{e}}\left(\frac{d q}{d \rho} v+2 q \frac{1}{\rho} v-2 \gamma \frac{u_{\rho}}{\rho^{2}} v\right) \rho^{2} d \rho=0,
\end{aligned}
$$

Integrando-se apenas a equação (6.35) por partes, chega-se à formulação fraca do problema dada por:

$$
\begin{aligned}
& \frac{d u}{d \rho}=q, \\
& \int_{\rho_{i}}^{\rho_{i+1}}\left(q \frac{d v}{d \rho} \rho^{2}+2 \gamma u v\right) d \rho=q v \rho^{2}\left(\rho_{i+1}^{-}\right)-q v \rho^{2}\left(\rho_{i}^{+}\right) .
\end{aligned}
$$

Aproximando-se a solução exata $(\mathrm{u}, \mathrm{q})$ com funções $\left(\mathrm{u}_{\mathrm{h}}, \mathrm{q}_{\mathrm{h}}\right)$ no espaço de elementos finitos $\mathrm{U}_{\mathrm{h}} \mathrm{xV}_{\mathrm{h}} \subset \mathrm{UxV}$ para qualquer $\mathrm{v} \in \mathrm{V}_{\mathrm{h}}$, tem-se que

$$
\begin{aligned}
& \frac{d u_{h}}{d \rho}=q_{h}, \\
& \int_{\rho_{i}}^{\rho_{i+1}}\left(q_{h} \frac{d v}{d \rho} \rho^{2}+2 \gamma u_{h} v\right) d \rho=\hat{q}_{h} v \rho^{2}\left(\rho_{i+1}^{-}\right)-\hat{q}_{h} v \rho^{2}\left(\rho_{i}^{+}\right),
\end{aligned}
$$

observa-se que nesta formulação aparece apenas o fluxo numérico $\hat{\mathrm{q}}_{\mathrm{h}}$, que são os mesmos adotados na formulação anterior, ou seja, para os elementos pertencentes ao interior do intervalo utiliza-se (6.25) e para os elementos da extremidade $\left(0, \rho_{\mathrm{e}}\right)$ utiliza-se $(6.26 \mathrm{~b})$ e (6.27b), respectivamente.

A solução aproximada $u_{h}$ adotada é dada por (6.28) e a solução aproximada $q_{h}$ é 
determinada a partir de (6.34) e é dada por

$$
\mathrm{q}_{\mathrm{h}}(\rho)=\frac{\mathrm{u}_{\mathrm{h}}\left(\rho_{\mathrm{i}+1}^{-}\right)-\mathrm{u}_{\mathrm{h}}\left(\rho_{\mathrm{i}}^{+}\right)}{\rho_{\mathrm{i}+1}-\rho_{\mathrm{i}}}
$$

De forma similar ao realizado na Seção 6.6, constroem-se três sistemas de equações lineares, sendo um para os elementos intermediários do intervalo $\left(0, \rho_{\mathrm{e}}\right), \mathrm{I}_{\mathrm{i}}, \mathrm{i}=2, \ldots, \mathrm{n}-1$, um para o elemento $I_{1}$ e um para o elemento $I_{n}$.

Para obter o sistema de equações dos elementos intermediários substitui-se $v=a+b \rho$, (6.28), (6.25) e (6.40) em (6.39). Por simplicidade, mostra-se abaixo, o sistema (6.38)-(6.39) com a substituição apenas da expressão $v=a+b \rho$

$$
\begin{aligned}
& \frac{d u_{h}}{d \rho}=q_{h}, \\
& \int_{\rho_{i}}^{\rho_{i+1}}\left(q_{h} b \rho^{2}+2 \gamma u_{h}(a+b \rho)\right) d \rho=\hat{q}_{h}(a+b \rho)^{2}\left(\rho_{i+1}^{-}\right)-\hat{q}_{h}(a+b \rho) \rho^{2}\left(\rho_{i}^{+}\right) .
\end{aligned}
$$

Fazendo-se todas as operações necessárias em (6.42) e agrupando-se os termos que possuem os coeficientes a e b em comum chega-se a uma equação esquematicamente representada por

$$
\mathrm{a}(\cdot)_{1}+\mathrm{b}(\cdot)_{2}=0
$$

Como os coeficientes a e b são arbitrários, implica que os termos (·) em (6.43) são nulos,

$$
(\cdot)_{1}=0, \quad(\cdot)_{2}=0,
$$


ou seja, obtém-se um sistema de duas equações lineares em cada elemento $I_{i}=\left(\rho_{i}^{+}, \rho_{i+1}^{-}\right)$, $\mathrm{i}=2, \ldots, \mathrm{n}-1$ para seis incógnitas (Fig. 6.5), a saber

i) $u_{h}\left(\rho_{i}^{-}\right), u_{h}\left(\rho_{i}^{+}\right)$pela esquerda e pela direita do nó $\rho_{\mathrm{i}}$, respectivamente;

ii) $\mathrm{u}_{\mathrm{h}}\left(\rho_{\mathrm{i}+1}^{-}\right), \mathrm{u}_{\mathrm{h}}\left(\rho_{\mathrm{i}+1}^{+}\right)$pela esquerda e pela direita do nó $\rho_{\mathrm{i}+1}$, respectivamente;

iii) $\mathrm{u}_{\mathrm{h}}\left(\rho_{\mathrm{i}-1}^{+}\right)$pela direta do nó $\rho_{\mathrm{i}-1}$;

iv) $u_{h}\left(\rho_{i+2}^{-}\right)$pela esquerda do nó $\rho_{i+2}$.

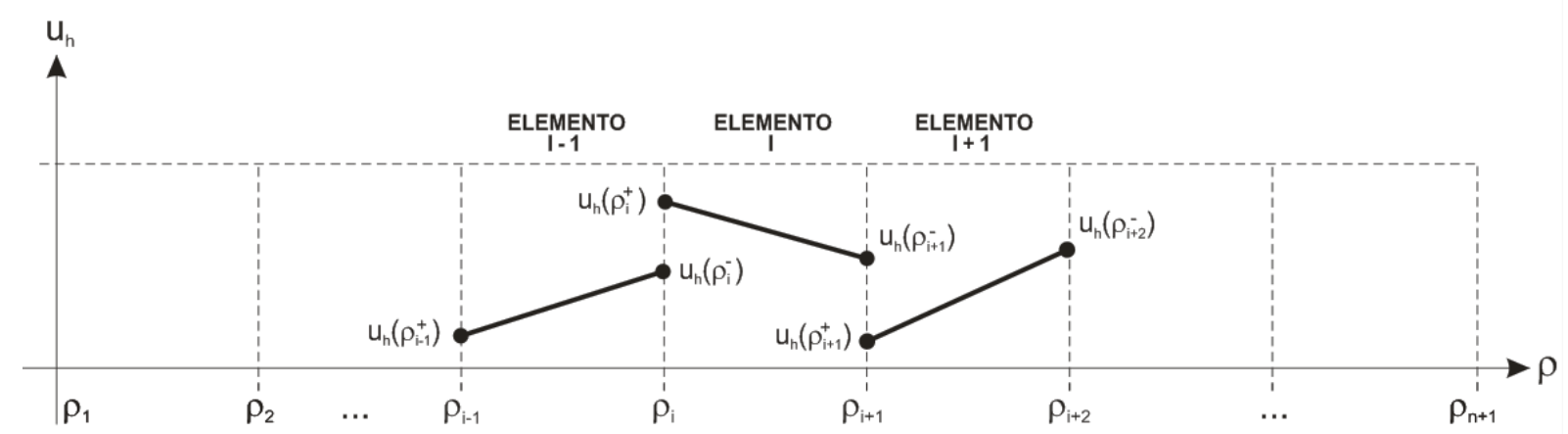

Figura 6.5. Graus de liberdade dos elementos intermediários na formulação alternativa do MEFGD.

Para obter o sistema de equações do elemento $I_{1}$ substitui-se $v=a+b \rho$, (6.28), (6.26b) e (6.40), em (6.39). Procedendo-se de maneira semelhante ao descrito para os elementos intermediários, obtém-se um sistema com duas equações e quatro incógnitas (Fig. 6.6) para o primeiro elemento, a saber

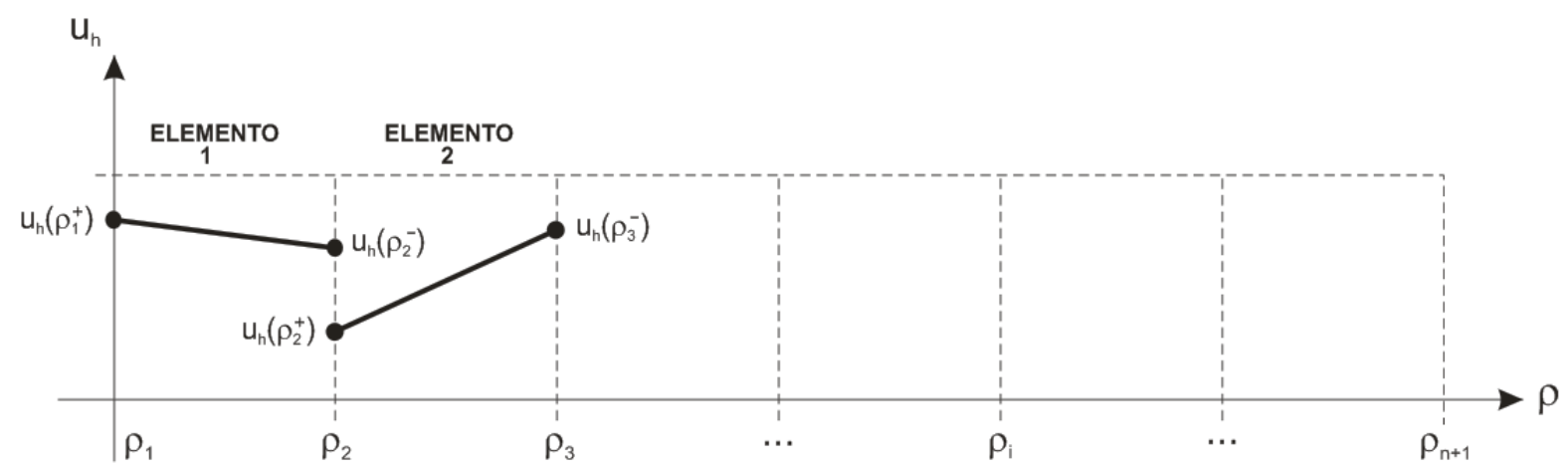

Figura 6.6. Graus de liberdade do elemento 1 na formulação alternativa do MEFGD.

i) $\mathrm{u}_{\mathrm{h}}\left(\rho_{1}^{+}\right)$pela direita do nó $\rho_{1}$; 
ii) $u_{h}\left(\rho_{2}^{-}\right)$pela esquerda do nó $\rho_{2}$;

iii) $\mathrm{u}_{\mathrm{h}}\left(\rho_{2}^{+}\right)$pela direita do nó $\rho_{2}$;

iv) $\mathrm{u}_{\mathrm{h}}\left(\rho_{3}^{-}\right)$pela esquerda do nó $\rho_{3}$.

Para obter o sistema de equações do elemento $I_{n}$ substitui-se $v=a+b \rho,(6.28)$, (6.27b) e (6.40), em (6.39). Semelhantemente ao descrito para os elementos intermediários, obtém-se um sistema com duas equações e quatro incógnitas (Fig. 6.7) para o último elemento, a saber

i) $u_{h}\left(\rho_{n-1}^{+}\right)$pela direita do nó $\rho_{n-1}$;

ii) $u_{h}\left(\rho_{n}^{-}\right)$pela esquerda do nó $\rho_{n}$;

iii) $\mathrm{u}_{\mathrm{h}}\left(\rho_{\mathrm{n}}^{+}\right)$pela direita do nó $\rho_{\mathrm{n}}$;

iv) $u_{h}\left(\rho_{n+1}^{-}\right)$pela esquerda do nó $\rho_{n+1}$.

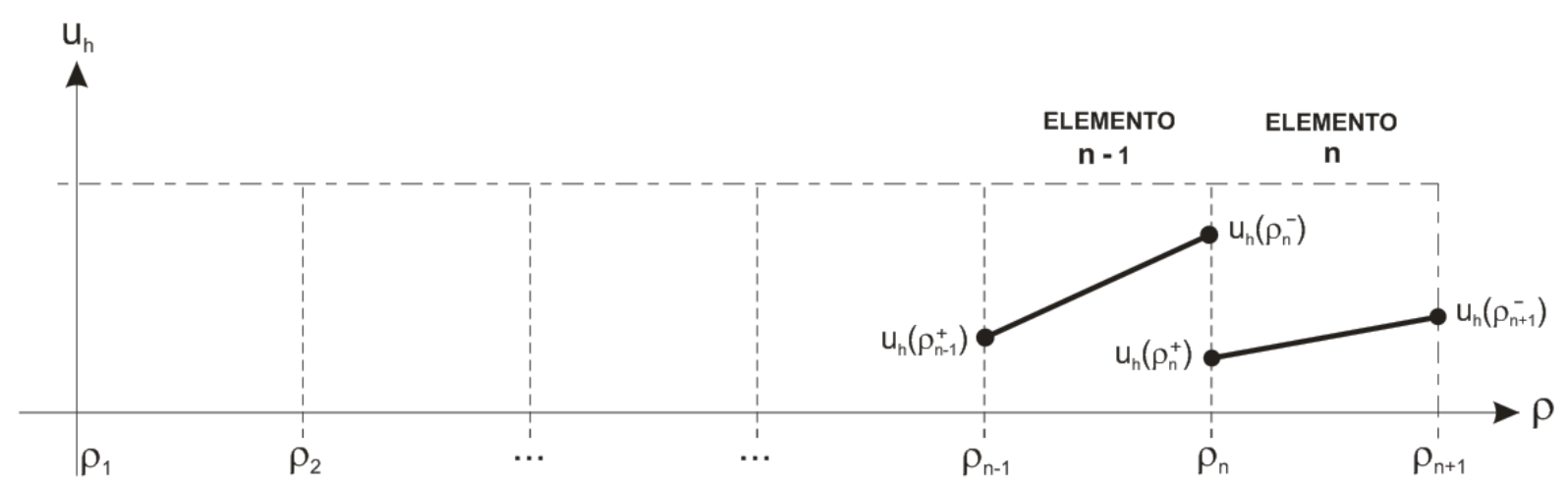

Figura 6.7. Graus de liberdade do elemento $n$ na formulação alternativa do MEFGD.

Observa-se das Figs. (6.5)-(6.7) que nesta formulação, associam-se a cada nó do interior do intervalo apenas os dois graus de liberdade em deslocamento, que correspondem aos valores do deslocamento pela direita e pela esquerda do nó. Sobre as extremidades do intervalo associa-se apenas um grau de liberdade que corresponde ao valor do deslocamento pelo lado interior do intervalo.

Sendo n o número de elementos utilizados na discretização do domínio, tem-se um sistema de $2 \mathrm{n}$ equações lineares com $2 \mathrm{n}$ incógnitas a serem determinadas. A solução deste 
sistema são os valores de $u_{h}$ à direita e à esquerda dos nós da malha utilizada. Os valores de $\mathrm{q}_{\mathrm{h}}$ são obtidos de (6.40).

Igualmente à formulação anterior, as aproximações $\mathrm{u}_{\mathrm{h}}$ e $\mathrm{q}_{\mathrm{h}}$ obtidas são descontínuas sobre os nós. Utilizam-se as definições $\hat{\mathrm{u}}=\mathrm{u}$ e $\hat{\mathrm{q}}=\mathrm{u}^{\prime}$ (CASTILLO, 2003) juntamente com as definições (6.24)-(6.27) para se obter as soluções aproximadas $u_{h}$ e $q_{h}$ sobre os nós. 


\section{$\mathbf{7}_{\text {Rentatate }}$}

\subsection{CONSIDERAÇÕES INICIAIS}

Apresentam-se os resultados numéricos gerados das soluções dos problemas discretos obtidos via MEFGC, MEFGD original e formulação alternativa do MEFGD descrita na Seção 6.7. Os resultados numéricos são comparados com a solução exata do problema.

Lembrando-se do Cap. 3 que o campo de deslocamento que satisfaz a condição de compatibilidade $u(0)=0$ do problema da esfera só existe para o parâmetro material $\kappa>1 / 3$, utiliza-se para a obtenção das soluções aproximadas os seguintes valores iniciais: $\kappa=0,4$ que fornece $\gamma=0,055$ de $(3.21), \quad p=1000, \quad \eta \equiv C_{12} / C_{11}=1 / 2, C_{11}=10^{5}, C_{22}=5 \times 10^{4}$ e $\mathrm{C}_{23}=5,5 \times 10^{3}$, em unidades adimensionais. Estes valores foram escolhidos de forma a satisfazer as restrições das constantes elásticas do material e dos valores da força compressiva p que podem ser aplicados na superfície externa da esfera, descritas no Cap. 3.

Primeiramente, apresentam-se os gráficos com os resultados numéricos obtidos utilizando-se diferentes malhas de elementos finitos para cada uma das formulações empregadas. Estas soluções aproximadas são comparadas com a solução exata do problema apresentada no Cap. 3. Gráficos comparativos entre as soluções aproximadas obtidas com o 
MEFGC e com ambas as formulações do MEFGD e curvas de erros entre as soluções aproximadas e a solução exata obtidas à medida que se refina a malha de elementos finitos para ambas as formulações são apresentadas. Por fim, analisa-se a convergência das soluções aproximadas de um ponto do domínio pertencente à região onde não ocorre a auto-intersecção da matéria e de outro pertencente à região onde ocorre a auto-intersecção.

\subsection{RESULTADOS OBTIDOS UTILIZANDO-SE O MEFGC}

Lembra-se da Seção 6.5 que a discretização do domínio foi feita com elementos finitos lineares por partes, contínuos sobre os nós. Nesta discretização cada elemento do domínio possui dois nós e um grau de liberdade por nó, resultando no total de dois graus de liberdade por elemento, sendo estes graus de liberdade de deslocamento.

\subsubsection{CAMPO DE DESLOCAMENTO}

Na Fig. 7.1 mostram-se os resultados para o campo de deslocamentos u versus o raio $\rho$ no intervalo $(0,1)$.

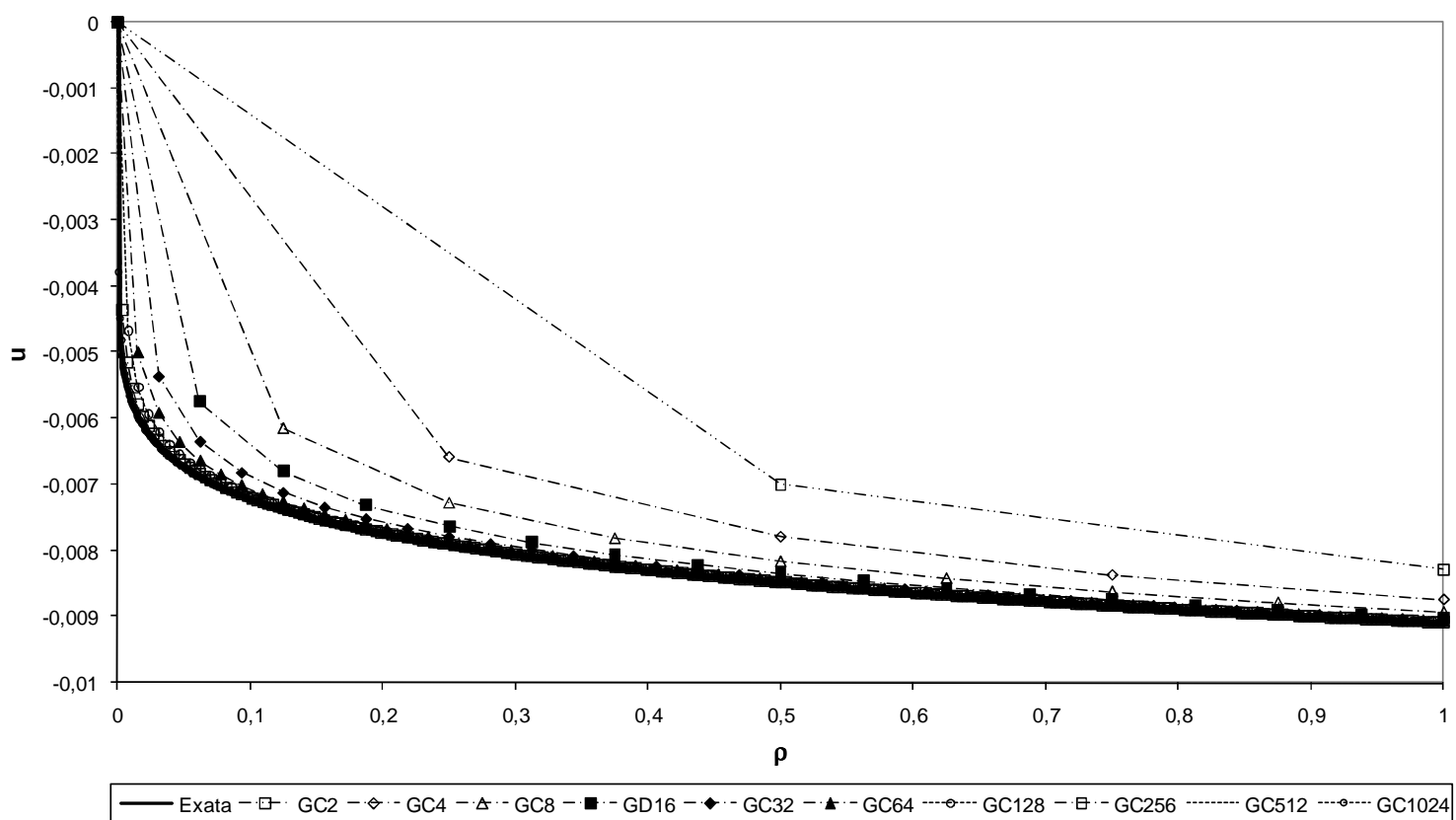

Figura 7.1. Solução exata u x solução $u_{h}$ com MEFGC para diferentes malhas. 
A linha cheia representa a solução exata dada por (3.26), (3.25), (3.24) e (3.21), e as demais linhas, tracejadas, representam soluções numéricas obtidas com o emprego do MEFGC para malhas uniformes com 2, 4, 8, 16, 32, 64, 128, 256, 512 e 1024 elementos. Observa-se deste gráfico que, à medida que se refina a malha de elementos finitos as soluções numéricas obtidas aproximam-se bem à solução exata do problema.

\subsubsection{CAMPO DE DEFORMAÇÃO}

A Fig. 7.2 mostra os resultados para o campo de deformação da esfera sob compressão radial uniformemente distribuída obtidos com o MEFGC para malhas uniformes com 2, 4, 8, $16,32,64,128,256,512$ e 1024 elementos, que são comparados com a solução exata do problema. O campo de deformação é uma grandeza derivada do campo de deslocamento. Uma vez que foram utilizadas funções lineares por partes para aproximar o campo de deslocamento, as aproximações obtidas para o campo de deformação são constantes sobre os elementos.

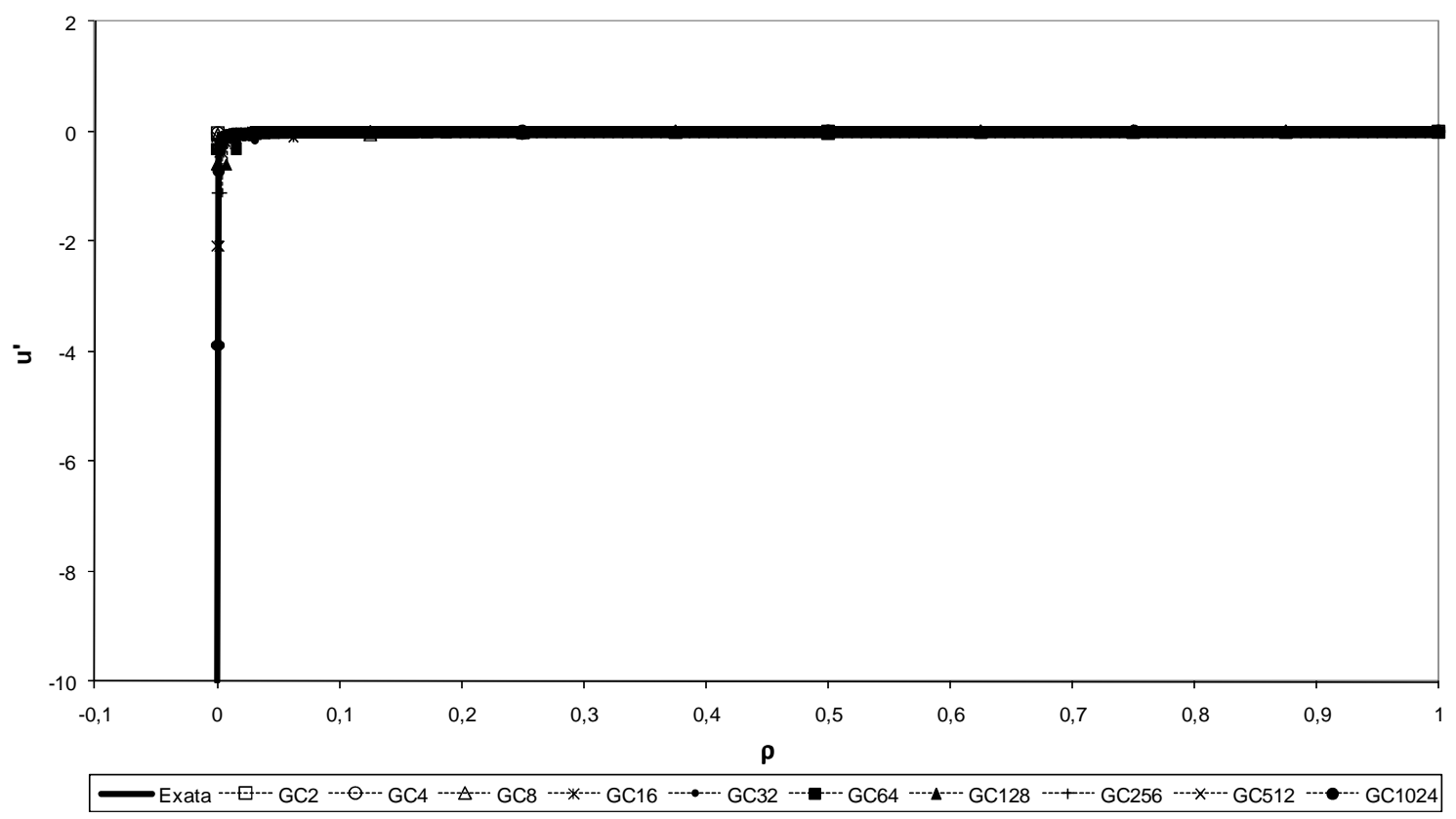

Figura 7.2. Solução exata u' x solução $\mathrm{q}_{\mathrm{h}}$ com MEFGC para diferentes malhas. 


\subsection{RESULTADOS OBTIDOS COM O MEFGD - FORMULAÇÃO ORIGINAL}

Para este estudo, lembra-se da Seção 6.6 que a discretização do domínio foi feita com elementos finitos lineares por partes e descontínuos sobre os nós. Nesta discretização cada elemento possui dois nós. Cada nó pertencente ao interior do intervalo $\left(0, \rho_{\mathrm{e}}\right)$ possui quatro graus de liberdade, sendo dois graus de liberdade de deslocamento, um pela esquerda e outro pela direita do nó, e dois graus de liberdade das derivadas, igualmente associados. Os nós das extremidades do intervalo possuem dois graus de liberdade, sendo um de deslocamento e um da derivada, definidos pelo interior do nó. O sistema resultante possui 4 n equações e $4 n$ incógnitas.

\subsubsection{CAMPO DE DESLOCAMENTO}

Na Fig. 7.3 mostram-se os resultados para o campo de deslocamentos u versus o raio $\rho$ no intervalo $(0,1)$.

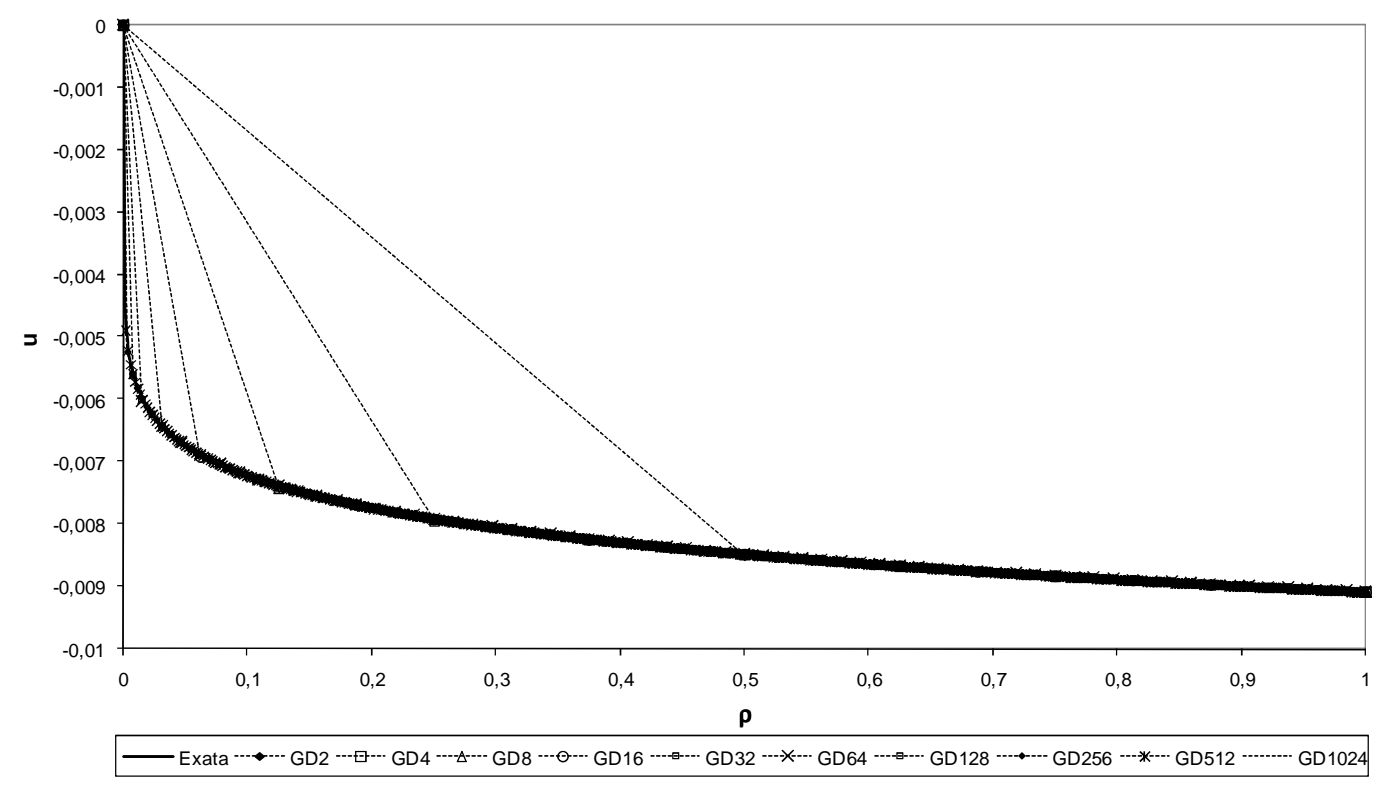

Figura 7.3. Solução exata u x solução $u_{h}$ com MEFGD original para diferentes malhas.

A linha cheia representa a solução exata dada por (3.26), (3.25), (3.24) e (3.21), e as 
demais linhas, tracejadas, representam soluções numéricas obtidas com o emprego da formulação original do MEFGD para malhas uniformes com 2, 4, 8, 16, 32, 64, 128, 256, 512 e 1024 elementos. Os resultados discretos obtidos à medida que se refina a malha de elementos finitos, fornecem boas aproximações para a solução exata do problema. Comparando-se a Fig. 7.1 com a Fig. 7.3 observa-se que na região do raio que varia de 0,5 a 1,0, o MEFGD fornece melhores aproximações para a solução exata do que o MEFGC.

\subsubsection{CAMPO DE DEFORMAÇÃO}

A Fig. 7.4 mostra os resultados para o campo de deformação da esfera obtido com o MEFGD para malhas uniformes com 2, 4, 8, 16, 32, 64, 128, 256, 512 e 1024 elementos, que são comparados com o resultado obtido da solução analítica do problema. Observa-se que esta formulação fornece boas aproximações para o campo de deformação da esfera.

Lembra-se da solução analítica do problema apresentada no Cap. 3 que quando $\rho=0$ o campo de deformação tende ao infinito. Observa-se da Fig. 7.4, na região próxima a $\rho=0$, que à medida que se refina a malha de elementos finitos os resultados numéricos obtidos com o MEFGD tendem gradativamente ao infinito.

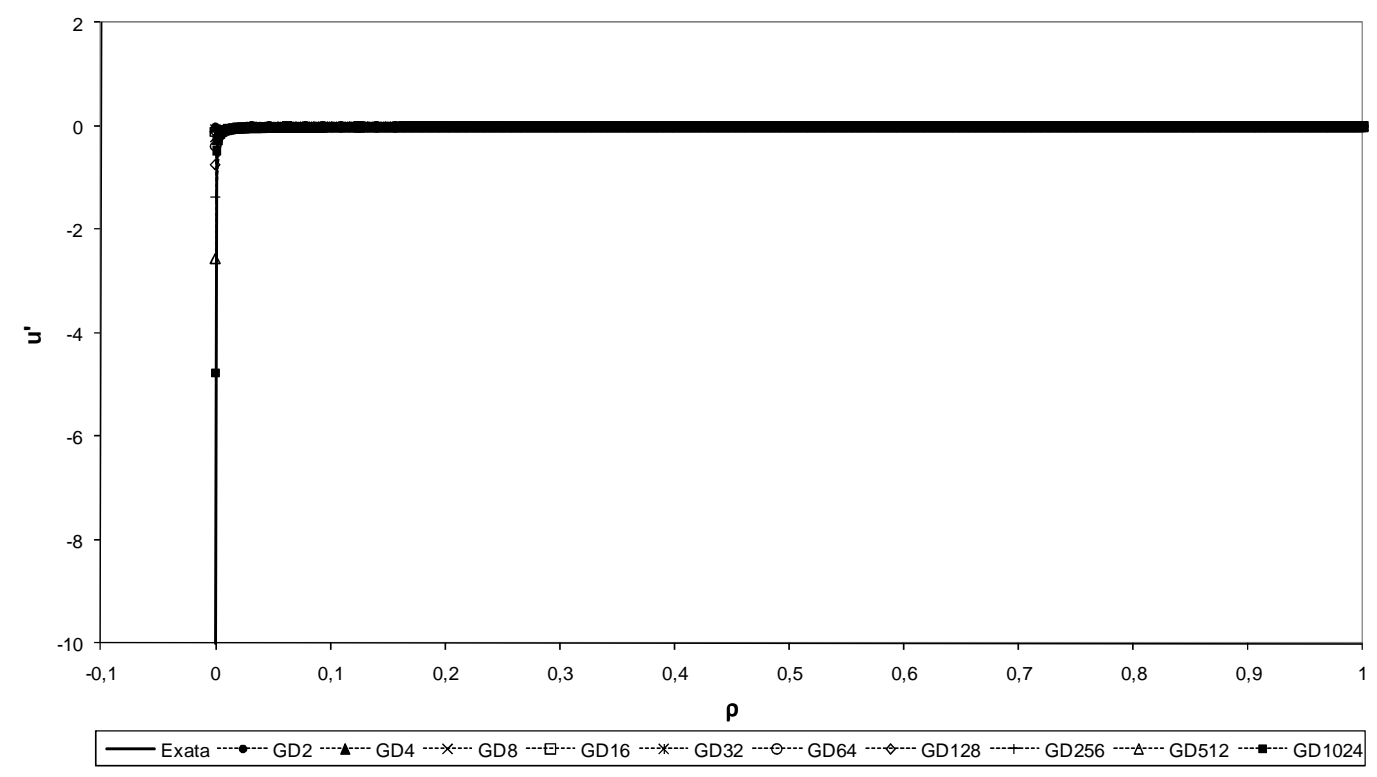

Figura 7.4. Solução exata u' x solução $\mathrm{q}_{\mathrm{h}}$ com MEFGD original para diferentes malhas. 


\subsection{RESULTADOS OBTIDOS COM O MEFGD - FORMULAÇÃO ALTERNATIVA}

Lembra-se da Seção 6.7 que nesta formulação, cada nó pertencente ao interior do intervalo $\left(0, \rho_{\mathrm{e}}\right)$ possui dois graus de liberdade, sendo dois graus de liberdade de deslocamento. Os nós da extremidade do intervalo possuem um grau de liberdade também de deslocamento. O sistema linear resultante possui 2 n equações e $2 n$ incógnitas.

\subsubsection{CAMPO DE DESLOCAMENTO}

Na Fig. 7.5 mostram-se os resultados para o campo de deslocamentos u versus o raio $\rho$ no intervalo $(0,1)$. A linha cheia representa a solução exata dada por (3.26), (3.25), (3.24) e (3.21), e as demais linhas, tracejadas, representam soluções numéricas obtidas com o emprego da formulação alternativa do MEFGD para malhas uniformes com 2, 4, 8, 16, 32, 64, 128, 256, 512 e 1024 elementos. A formulação fornece boas aproximações para a solução exata do problema à medida que se refina a malha de elementos finitos.

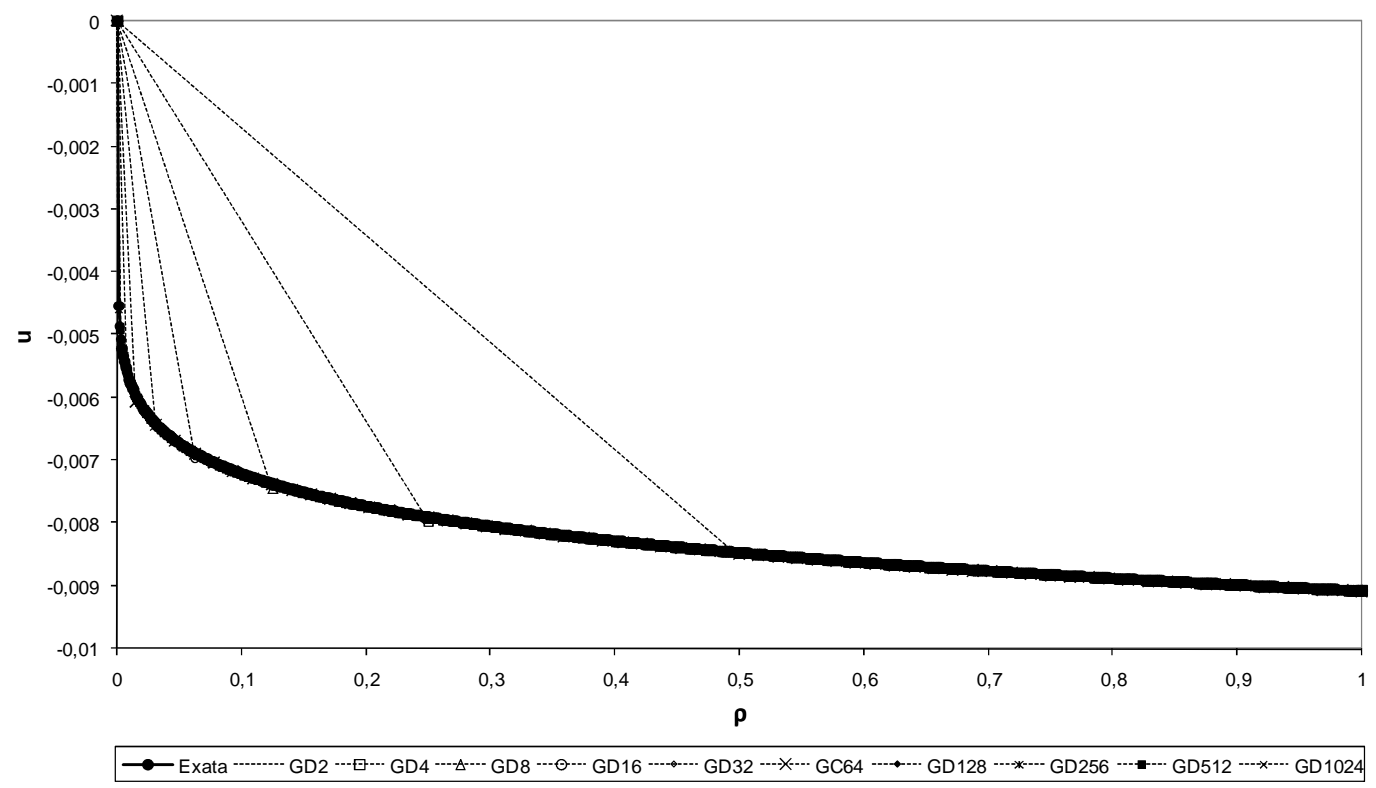

Figura 7.5. Solução exata u x solução $\mathrm{u}_{\mathrm{h}}$ com MEFGD alternativo para diferentes malhas. 


\subsubsection{CAMPO DE DEFORMAÇÃO}

A Fig. 7.6 mostra os resultados para o campo de deformação da esfera obtidos com a formulação alternativa do MEFGD para malhas uniformes com 2, 4, 8, 16, 32, 64, 128, 256, 512 e 1024 elementos, comparados com a solução exata do problema. Nesta formulação o campo de deformação é derivado do campo de deslocamento $\mathrm{u}_{\mathrm{h}} \quad$ (Seção 6.7) semelhantemente ao MEFGC e não diretamente da inversão do sistema de equações resultante como na formulação original do MEFGD, onde a aproximação é linear. Desta forma, as aproximações obtidas são constantes no interior de cada elemento. Observa-se que no limite do refinamento, as aproximações constantes sobre os elementos próximos à origem tendem ao infinito.

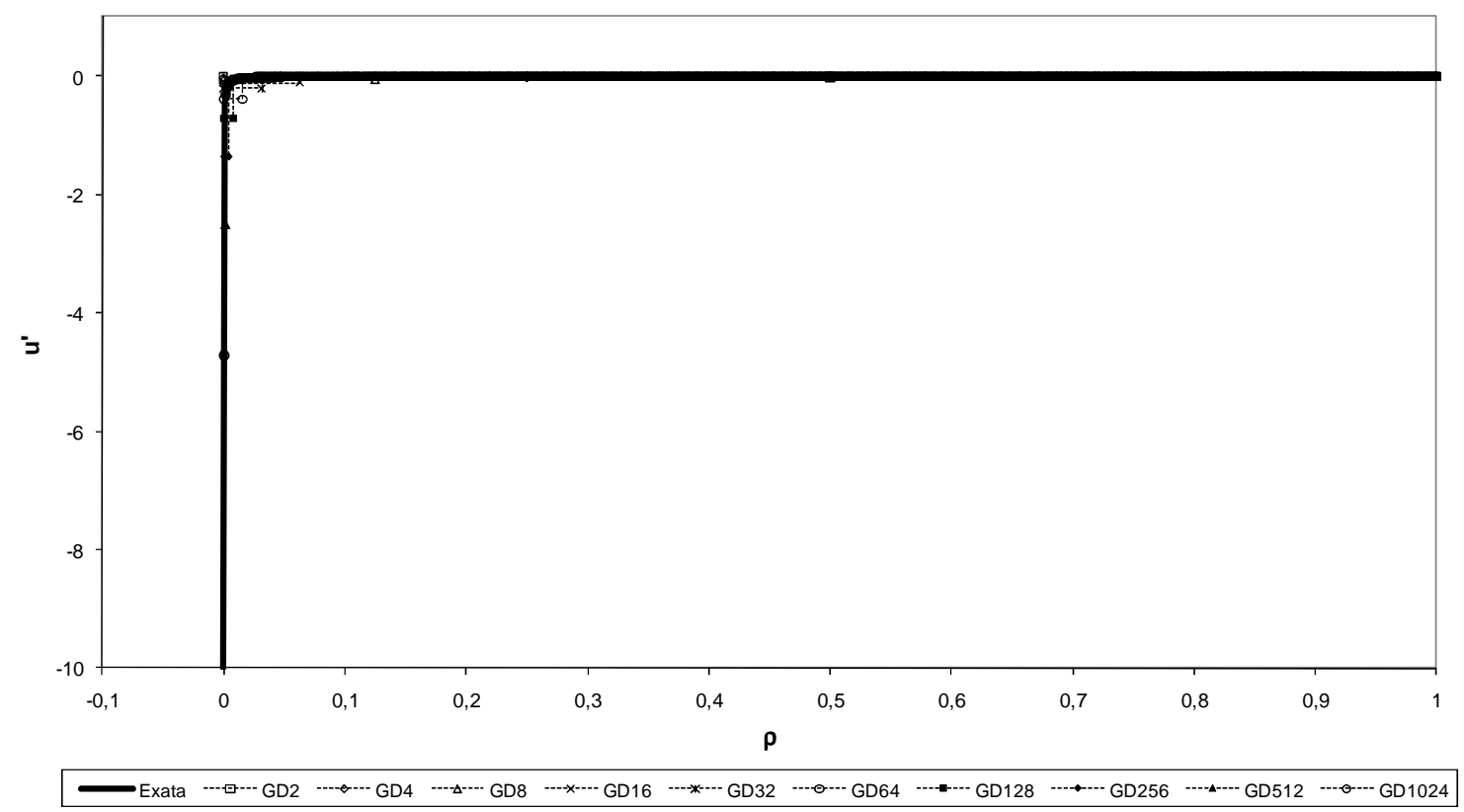

Figura 7.6. Solução exata u' x solução $\mathrm{q}_{\mathrm{h}}$ com MEFGD alternativo para diferentes malhas. 


\subsection{COMPARAÇÃO DOS RESULTADOS}

\subsubsection{CAMPO DE DESLOCAMENTO}

Na Fig. 7.7 mostra-se o gráfico do campo de deslocamento u versus o raio $\rho$ no intervalo $(0,1)$. A linha cheia representa a solução exata dada por (3.26), (3.25), (3.24) e (3.21), e as demais linhas, tracejadas, representam soluções numéricas obtidas com o emprego do MEFGC, MEFGD original e MEFGD alternativo para uma malha uniforme com 1024 elementos. Observa-se deste gráfico que todas as soluções numéricas aproximam bem a solução exata.

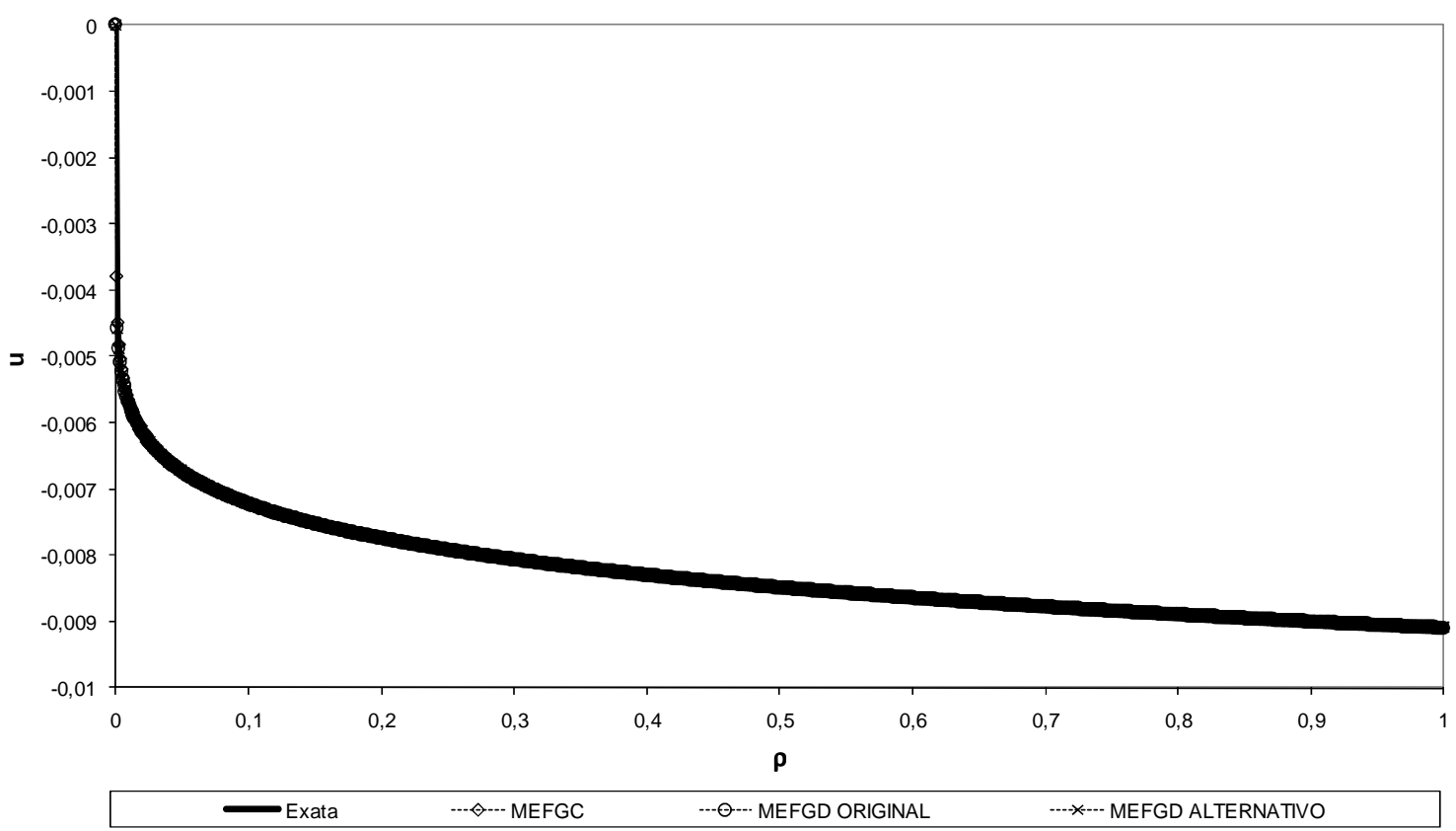

Figura 7.7. Curvas de deslocamento $\mathrm{u}$ e $\mathrm{u}_{\mathrm{h}} \mathrm{x}$ raio $\rho$ no intervalo $(0,1)$.

\subsubsection{CAMPO DE DEFORMAÇÃO}

Na Fig. 7.8 mostra-se o campo de deformação $\varepsilon_{\rho \rho}=u^{\prime}$ versus o raio $\rho \in(0,1)$.

Similarmente ao exposto acima, a linha cheia representa a expressão exata de $\mathrm{u}^{\prime}$ e as demais 
linhas representam soluções numéricas obtidas com o emprego do MEFGC, MEFGD original e MEFGD alternativo para uma malha uniforme com 1024 elementos. Observa-se do exposto nas Seções 6.5 e 6.7 que as aproximações de $u^{\prime}$ obtidas de ambos, MEFGC e MEFGD alternativo, são constantes sobre cada elemento e que as aproximações de $\mathrm{u}^{\prime}$ obtidas do MEFGD original são lineares sobre cada elemento. Observa-se deste gráfico que todas as expressões numéricas aproximam bem a expressão exata de $\mathrm{u}^{\prime}$. Observa-se ainda que as aproximações obtidas com todas as formulações tendem ao infinito à medida que $\rho$ tende a zero.

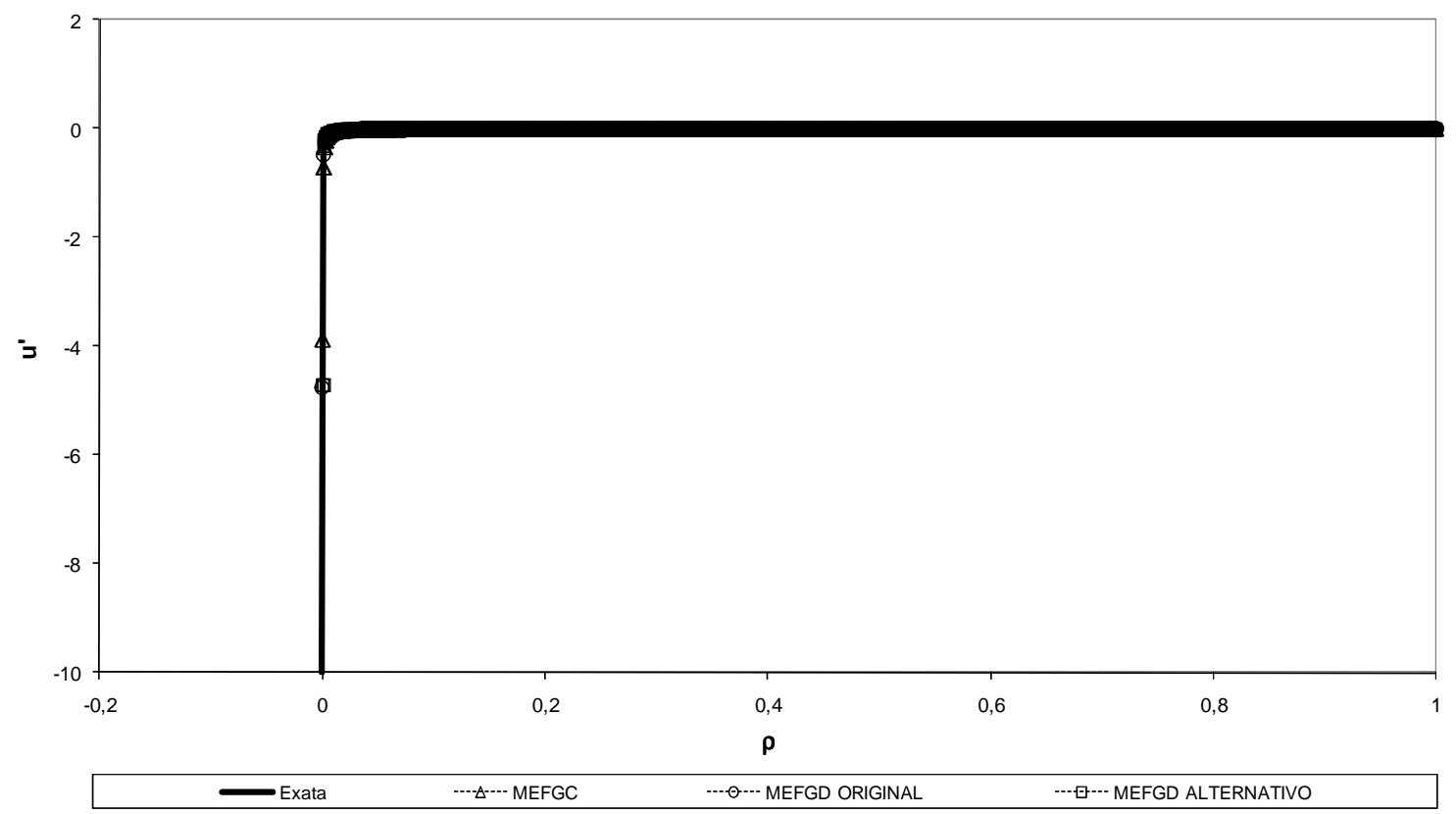

Figura 7.8. Curvas de deformação u' e $\mathrm{q}_{\mathrm{h}} \mathrm{x}$ raio $\rho$ no intervalo $(0,1)$.

\subsection{ANÁLISE DE ERROS}

\subsubsection{CAMPO DE DESLOCAMENTO}

A Fig. 7.9 mostra curvas de erros entre a solução exata u e as soluções aproximadas $\mathrm{u}_{\mathrm{h}}$ geradas pelas formulações do MEFGC, MEFGD original e MEFGD alternativo, utilizando-se malhas uniformes com 2, 4, 8, 16, 32, 64, 128, 256, 512 e 1024 elementos. Para 
o cálculo destes erros utilizou-se a norma Euclidiana

$$
\|\mathrm{e}\|_{2}=\left(\sum_{\mathrm{i}=1}^{\mathrm{n}}\left|\mathrm{e}_{\mathrm{i}}\right|^{2}\right)^{1 / 2},
$$

onde $e_{i}$ é o erro da aproximação por elementos finitos definido por

$$
\mathrm{e}_{\mathrm{i}}=\mathrm{u}\left(\rho_{\mathrm{i}}\right)-\hat{\mathrm{u}}_{\mathrm{h}}\left(\rho_{\mathrm{i}}\right)
$$

Para obtenção dos erros com o MEFGC, os valores nodais $u_{h}$ obtidos da inversão do sistema de equações resultante da substituição de (6.7)-(6.8) em (6.11) foram substituídos em (7.2) e estes, em (7.1) para cada uma das discretizações utilizadas. Para obtenção dos erros com ambas as formulações do MEFGD, uma vez que as aproximações $\mathrm{u}_{\mathrm{h}}$ obtidas nestas formulações são descontínuas sobre os nós, utilizou-se a definição $\mathrm{u}=u$ (CASTILLO, 2003) juntamente com as definições (6.24), (6.26a) e (6.27a) para se obter as soluções aproximadas $\mathrm{u}_{\mathrm{h}}$ sobre os nós. Com estas aproximações aplicou-se a definição (7.2) em (7.1) para obter os erros para cada uma das discretizações utilizadas.

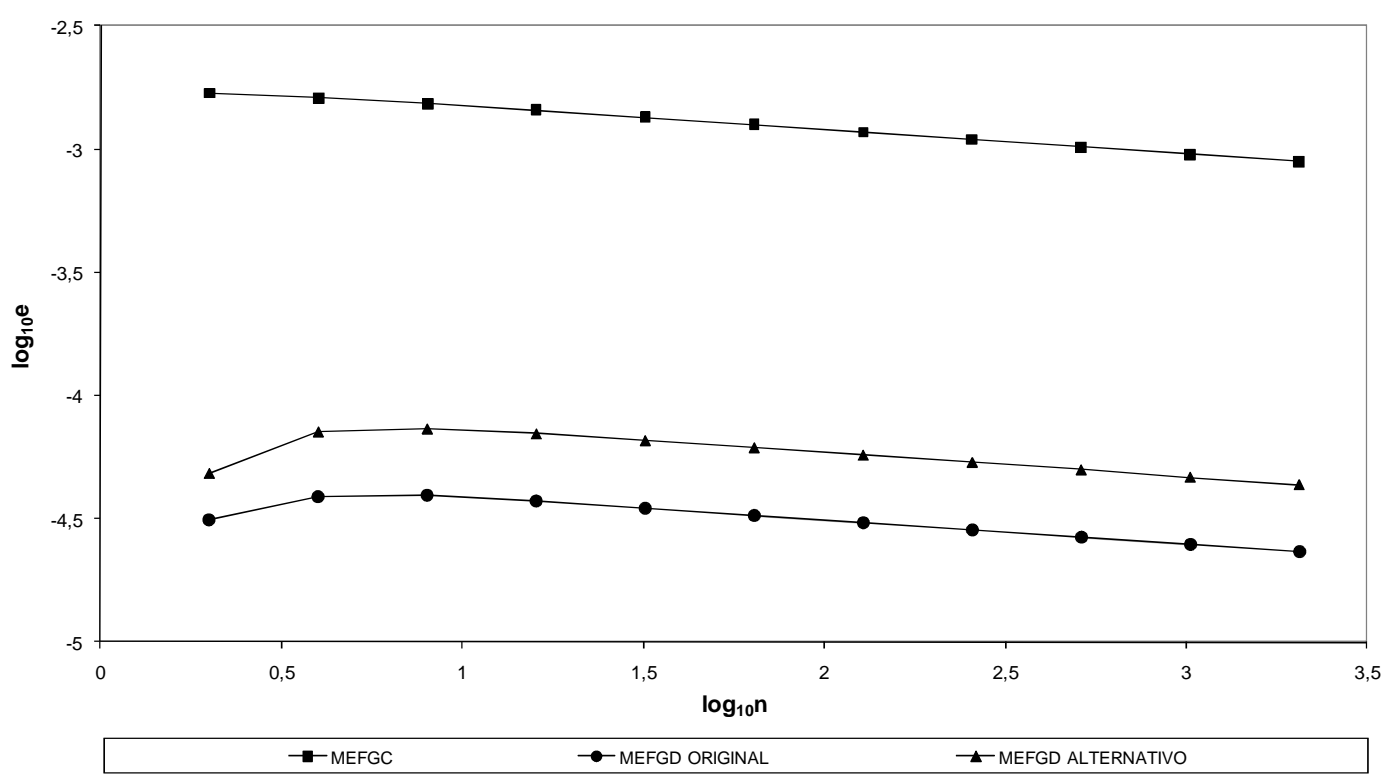

Figura 7.9. Curvas de erro obtidas de resultados gerados pelo MEFGC, MEFGD original e MEFGD alternativo. 
As curvas mostradas na Fig. 7.9 referem-se ao logaritmo na base dez do erro e dado por (7.1) versus o logaritmo na base dez do número de elementos finitos utilizados para discretizar o domínio em todos os métodos. Observa-se destas curvas que os resultados gerados pelas formulações original e alternativa do MEFGD são bem mais precisos que os resultados gerados pelo MEFGC. Observa-se ainda que os erros obtidos com a formulação alternativa do MEFGD são um pouco maiores que os erros obtidos com a formulação original do MEFGD.

\subsubsection{CAMPO DE DEFORMAÇÃO}

A Fig. 7.10 mostra curvas de erros entre a solução exata $u^{\prime}$ e as soluções aproximadas $\mathrm{q}_{\mathrm{h}}$ geradas pelas formulações do MEFGC, MEFGD original e MEFGD alternativo, utilizando-se malhas uniformes com 2, 4, 8, 16, 32, 64, 128, 256, 512 e 1024 elementos. Para o cálculo destes erros utilizou-se a definição de erro médio

$$
\mathrm{e}_{\mathrm{m}}=\frac{1}{\mathrm{n}+1} \sum_{\mathrm{j}=1}^{\mathrm{n}+1}\left|\mathrm{e}_{\mathrm{j}}\right|
$$

onde $n$ é o número de elementos finitos e $e_{j}=u^{\prime}\left(\rho_{j}\right)-\hat{q}_{h}\left(\rho_{j}\right)$.

Para obtenção dos erros com o MEFGC, os valores nodais $\mathrm{q}_{\mathrm{h}}$ obtidos a partir dos valores de $u_{h}$ foram substituídos em $e_{j}=u^{\prime}\left(\rho_{j}\right)-\hat{q}_{h}\left(\rho_{j}\right)$ e estes, em (7.3) para cada uma das discretizações utilizadas. Para obtenção dos erros com ambas as formulações do MEFGD, uma vez que as aproximações $\mathrm{q}_{\mathrm{h}}$ obtidas nestas formulações são descontínuas sobre os nós, utilizou-se a definição $\hat{\mathrm{q}}=\mathrm{u}^{\prime}$ (CASTILLO, 2003) juntamente com as definições (6.25), (6.26b) e (6.27b) para se obter as soluções aproximadas $\mathrm{q}_{\mathrm{h}}$ sobre os nós e aplicou-se a 
definição (7.2) em (7.3) para cada uma das discretizações utilizadas.

As curvas mostradas na Fig. 7.10 referem-se ao logaritmo na base dez do erro médio dado por (7.3) versus o logaritmo na base dez do número de elementos finitos utilizados para discretizar o domínio. Observa-se destas curvas que, à medida que se refina a malha de elementos finitos, o erro médio diminui para todas as formulações. Para uma dada malha, os resultados gerados pela formulação original do MEFGD são mais precisos que os resultados gerados pela formulação alternativa do MEFGD. Além disso, os erros obtidos com ambas as formulações do MEFGD são bem inferiores àqueles obtidos com o MEFGC.

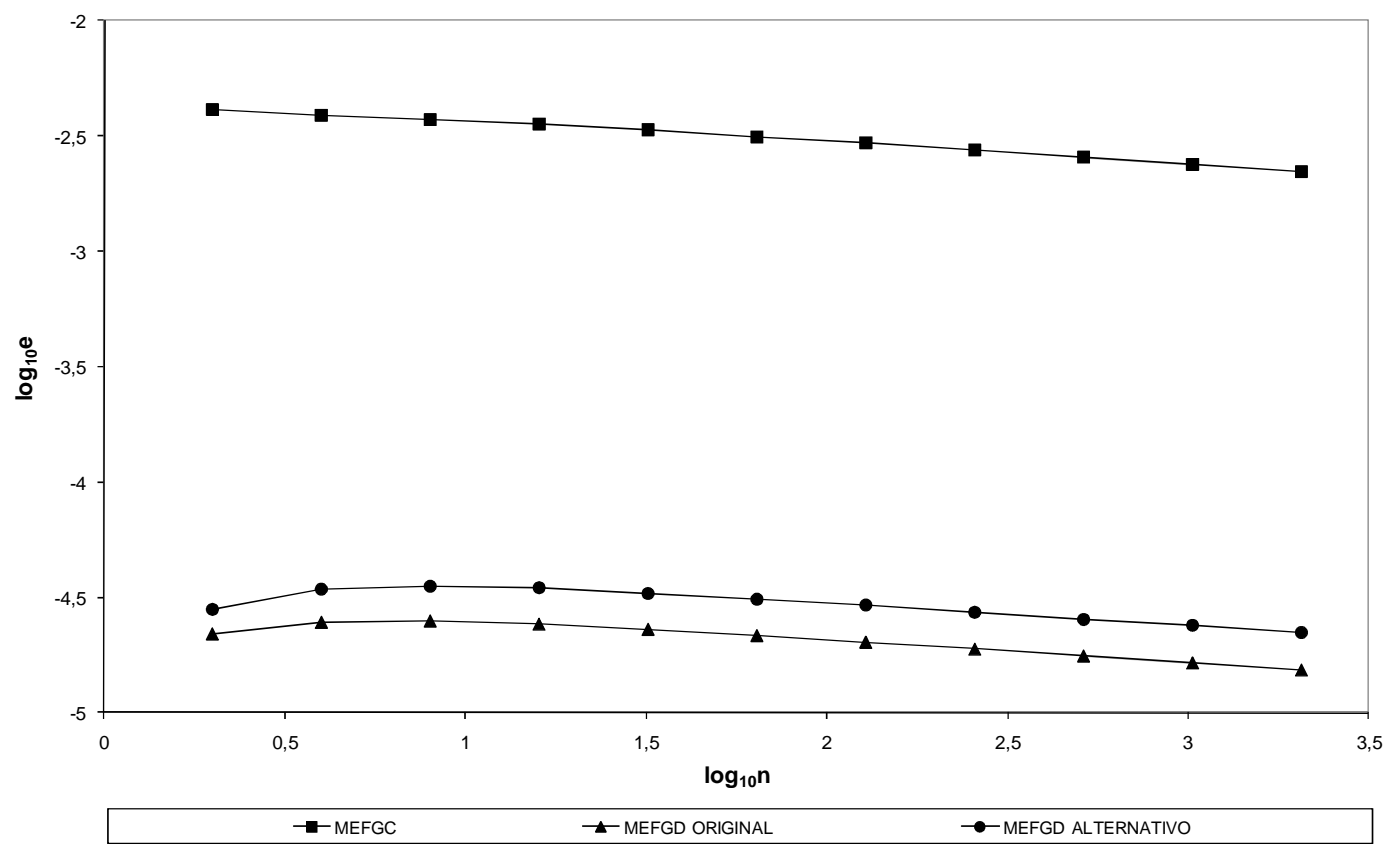

Figura 7.10. Curvas de erro obtidas de resultados gerados pelo MEFGC, MEFGD original e MEFGD alternativo.

\subsection{ANÁLISE DE CONVERGÊNCIA DOS MÉTODOS EM PONTOS SITUADOS DENTRO E FORA DA REGIÃO DE AUTO-INTERSECÇÃO}

Considerando-se que o problema da esfera possui duas regiões, uma onde ocorre e outra onde não ocorre o fenômeno da auto-intersecção, com características bem diferentes, analisa-se a convergência das soluções aproximadas obtidas para um ponto pertencente a cada 
uma destas regiões com ambas as formulações dos métodos dos elementos finitos empregadas.

As soluções aproximadas podem convergir para a solução exata de um problema de forma monótona ou não monótona (SORIANO, 2003).

Seja a função erro definida por (7.2), diz-se que as soluções aproximadas obtidas à medida que se refina a malha de elementos finitos, convergem de forma monótona para a solução exata do problema quando a variação da função erro tende a zero monotonicamente, caso contrário, a convergência é não monótona (Fig. 7.11).

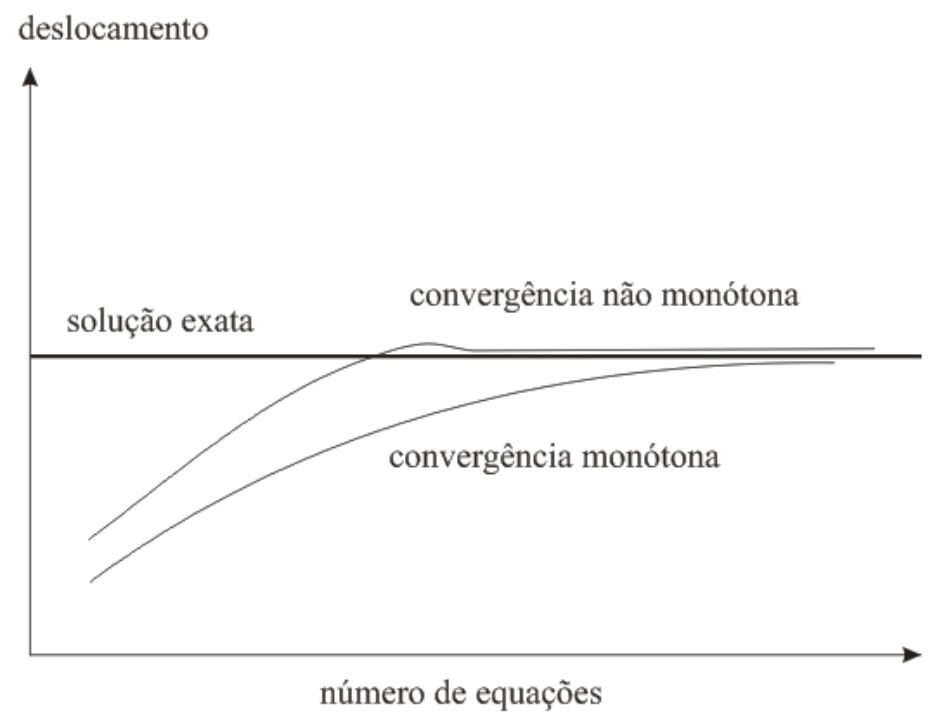

Figura 7.11. Curvas de convergência monótona e não monótona (Fonte: Soriano, 2003).

\subsubsection{CAMPO DE DESLOCAMENTO}

Mostra-se na Fig. 7.12 um gráfico do deslocamento calculado em $\rho=1$ versus o logaritmo na base 2 do número de elementos finitos. A linha cheia horizontal corresponde ao valor exato deste deslocamento e as outras linhas correspondem aos valores aproximados obtidos com o MEFGC, MEFGD original e MEFGD alternativo. Observa-se desta figura que os deslocamentos nodais obtidos com o MEFGC convergem assintoticamente para o 
deslocamento nodal obtido da solução exata à medida que se refina a malha de elementos finitos utilizada para discretizar o domínio. Os deslocamentos nodais obtidos com ambas as formulações do MEFGD aproximam melhor os deslocamentos nodais obtidos da solução exata do que os deslocamentos nodais obtidos com o MEFGC. Em particular, observa-se que para uma discretização com apenas dois elementos ambas as formulações do MEFGD produzem aproximações à solução exata que são sensivelmente melhores do que a aproximação produzida com dois elementos do MEFGC. Observa-se ainda que a convergência do MEFGC é monótona conforme predito na literatura para métodos de elementos finitos conformes (SORIANO, 2003).

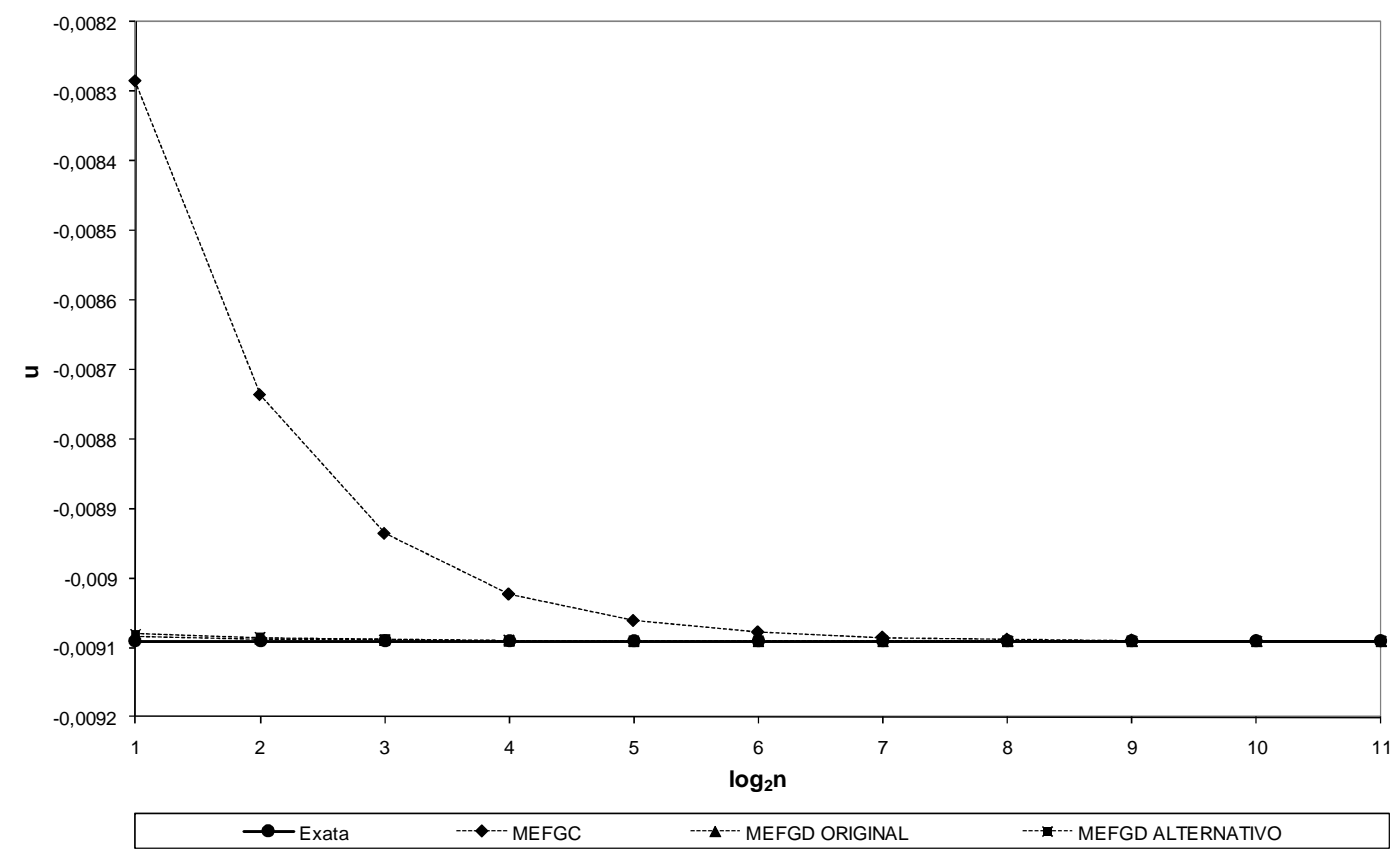

Figura 7.12. Curvas deslocamento $\mathrm{x} \log _{2} \mathrm{n}$ para ponto na região sem auto-intersecção.

Mostra-se na Fig. 7.13 um gráfico do deslocamento calculado em $\rho=1 / 256$ versus o logaritmo na base 2 do número de elementos finitos. A linha cheia horizontal corresponde ao valor exato deste deslocamento e as outras linhas correspondem aos valores aproximados obtidos com o MEFGC, MEFGD original e MEFGD alternativo. Observa-se da Fig. 7.13 que a curva obtida com o MEFGC indica tendência de convergência assintótica para a solução exata. Os deslocamentos nodais obtidos para ambas as formulações do MEFGD convergem 
para os deslocamentos nodais obtidos da solução exata. Os erros obtidos com ambas as formulações do MEFGD são bem menores do que os erros obtidos com o MEFGC.

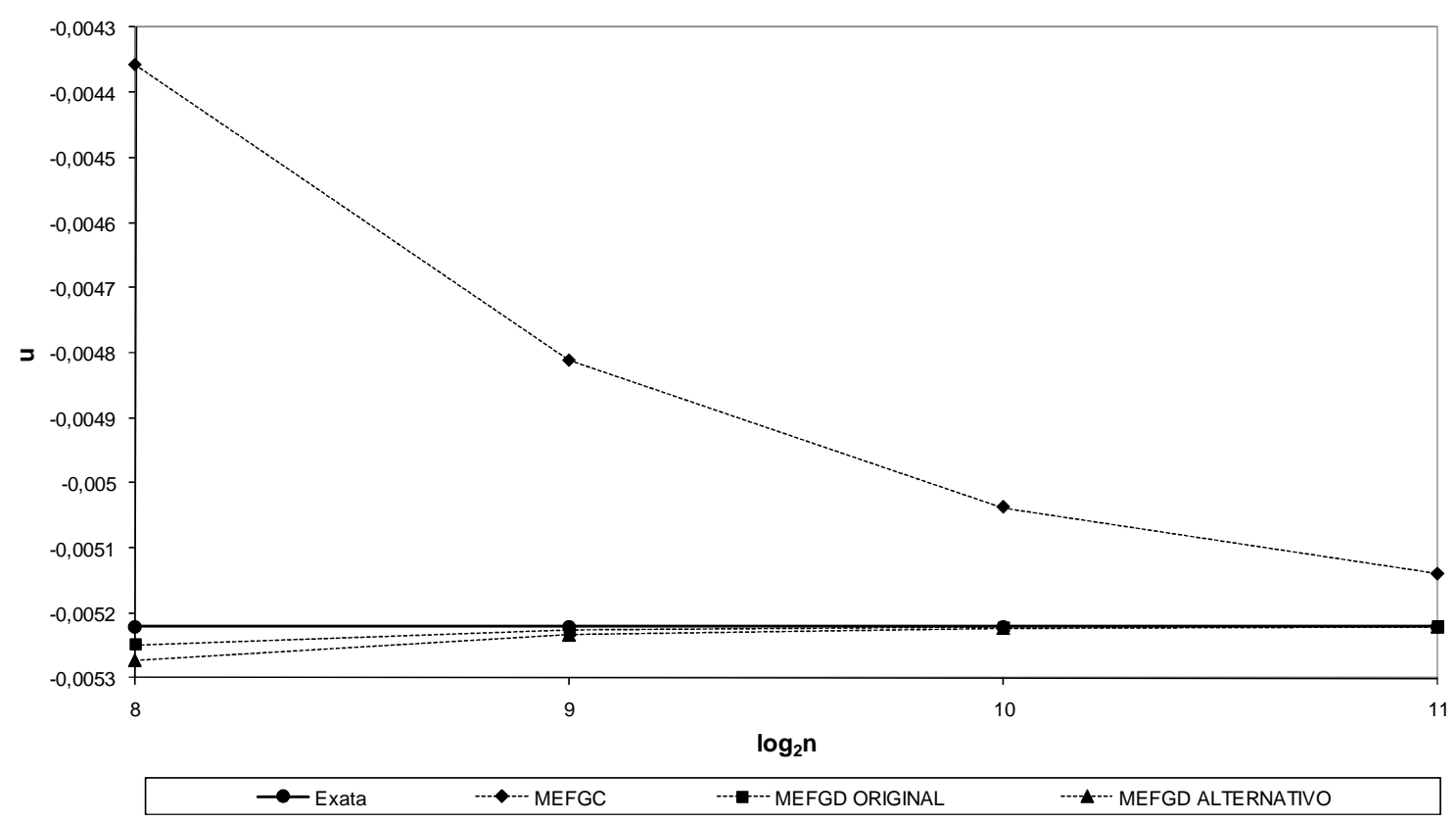

Figura 7.13. Curvas deslocamento $\mathrm{x} \log _{2} \mathrm{n}$ para ponto na região com auto-intersecção.

A Fig. 7.14 mostra uma ampliação da Fig. 7.13 na vizinhança do valor exato de $\mathrm{u}(1 / 256)$ para as soluções aproximadas obtidas com ambas as formulações do MEFGD. Observa-se que ambas convergem de forma não monótona para a solução exata do problema.

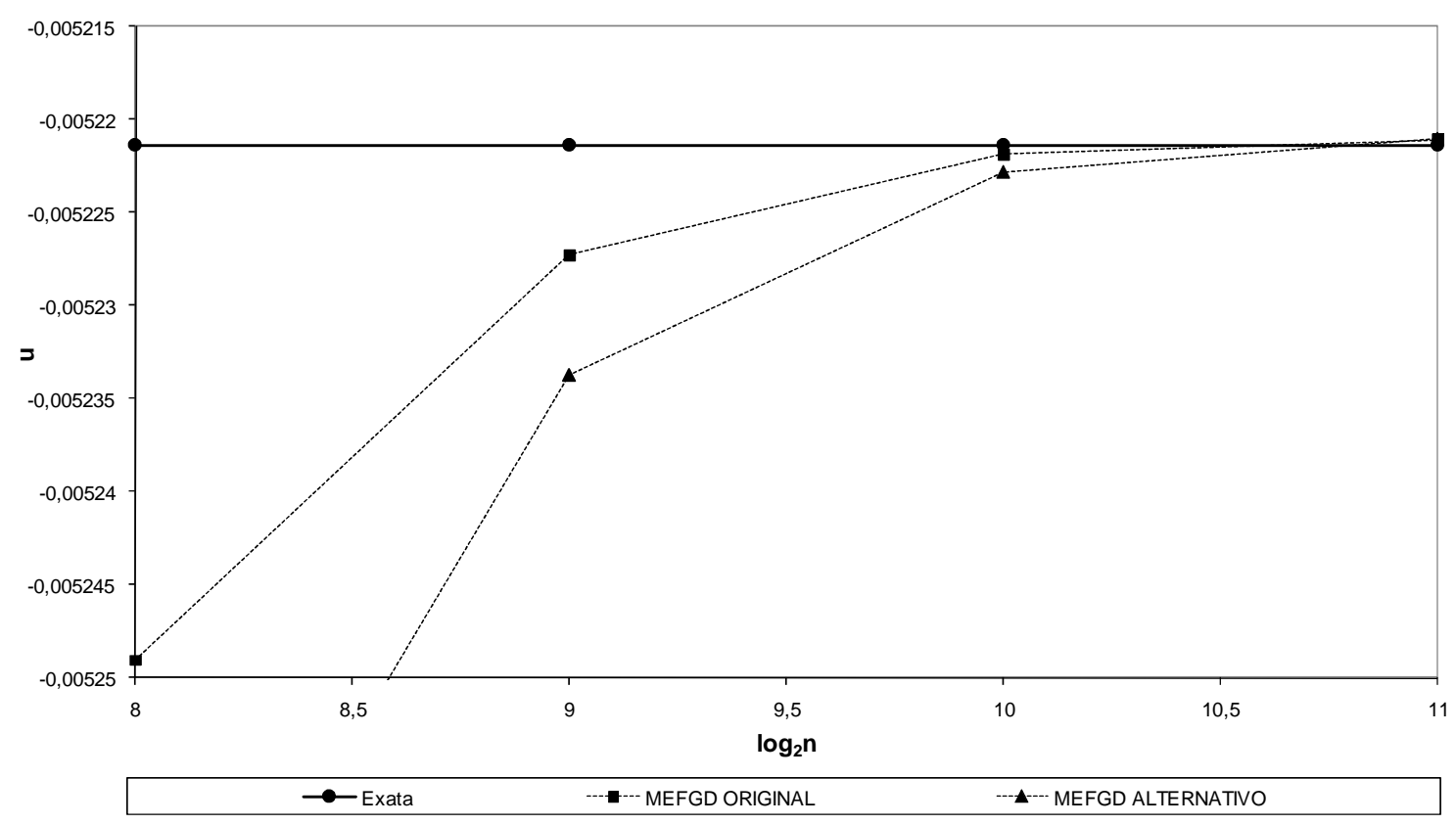

Figura 7.14. Curvas de deslocamento obtidas com MEFGD original e MEFGD alternativo. 


\subsubsection{CAMPO DE DEFORMAÇÃO}

Mostra-se na Fig. 7.15 um gráfico da deformação calculada em $\rho=1$ versus o logaritmo na base 2 do número de elementos finitos. A linha cheia horizontal corresponde ao valor exato desta deformação e as outras linhas, tracejadas, correspondem aos valores aproximados obtidos com o MEFGC, MEFGD original e MEFGD alternativo.

Observa-se desta figura que as deformações obtidas das soluções aproximadas pelo MEFGC convergem assintoticamente para o valor exato de deformação e que ambas as formulações do MEFGD fornecem aproximações mais precisas do que as aproximações obtidas com o MEFGC.

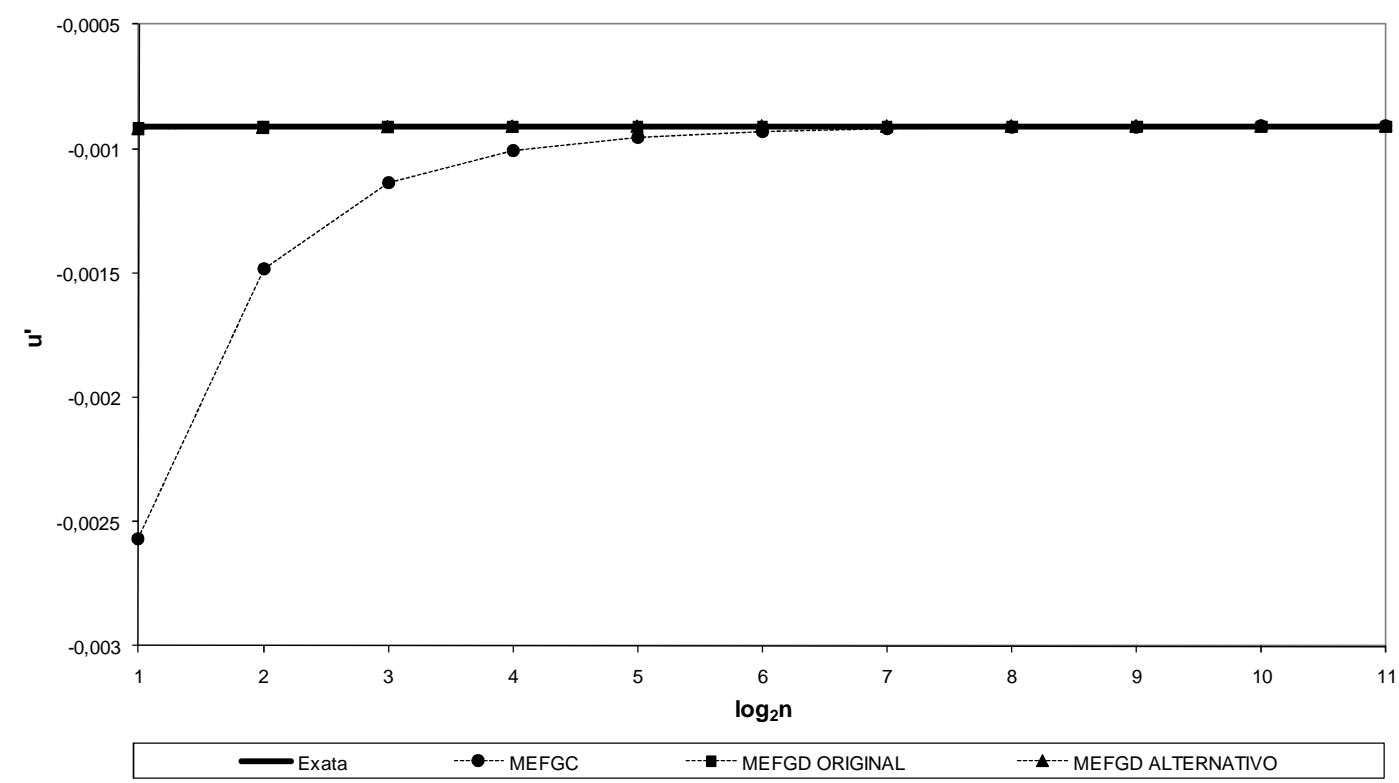

Figura 7.15. Curvas deformação $\mathrm{x} \log _{2} \mathrm{n}$ para ponto na região sem auto-intersecção.

A Fig. 7.16 mostra uma ampliação da Fig. 7.15 na vizinhança do valor exato de u'(1) para destacar as curvas das deformações aproximadas obtidas com ambas as formulações do MEFGD. Observa-se que as deformações nodais obtidas com ambas as formulações do MEFGD convergem assintoticamente para o deslocamento nodal obtido da solução exata do problema à medida que se refina a malha de elementos finitos. As aproximações obtidas com 
a formulação original do MEFGD são melhores do que as aproximações obtidas com a formulação alternativa do MEFGD.

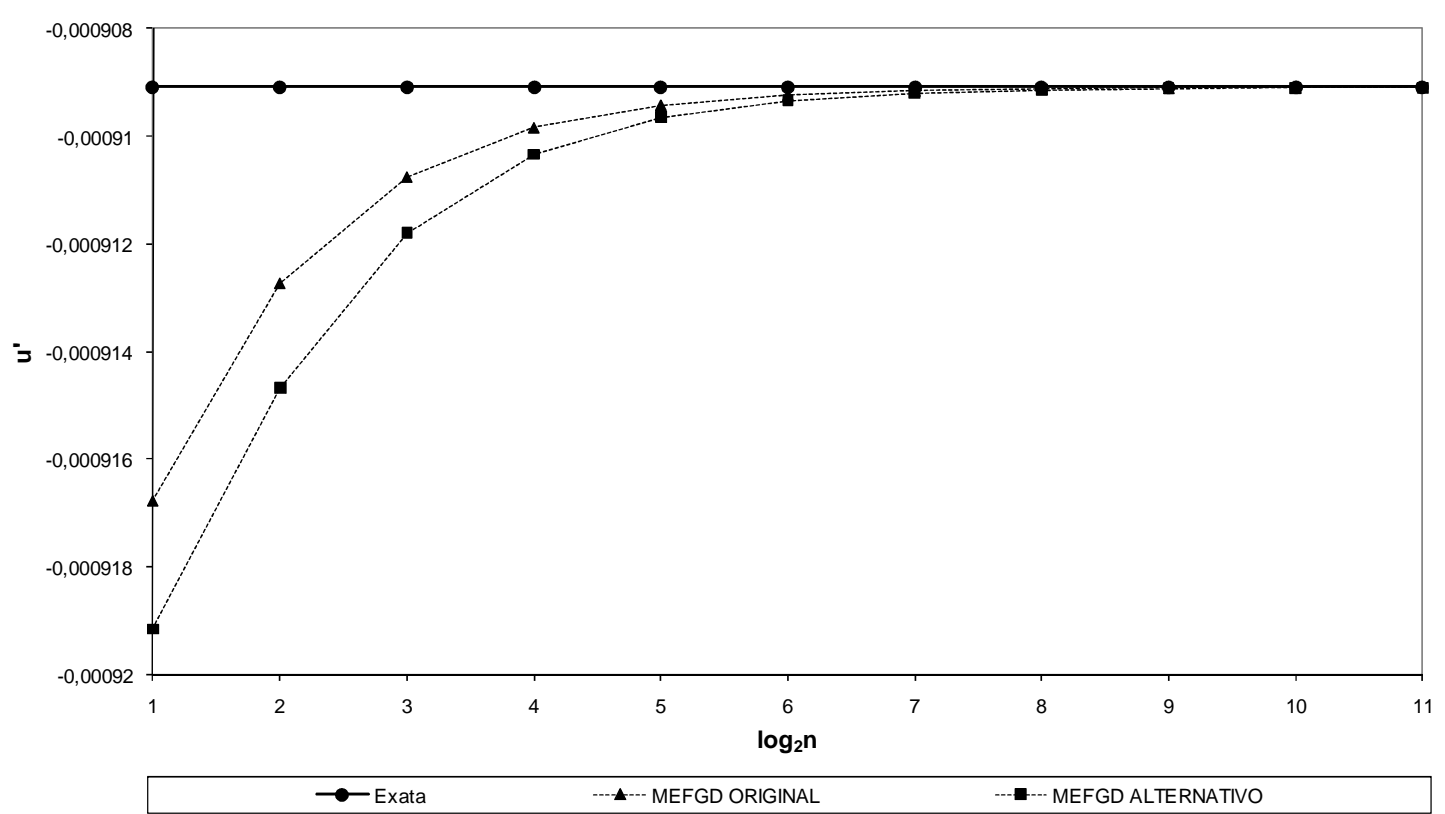

Figura 7.16. Curvas deformação $\mathrm{x} \log _{2} \mathrm{n}$ ampliadas obtidas com MEFGD original e MEFGD alternativo.

Mostra-se na Fig. 7.17 um gráfico da deformação calculada em $\rho=1 / 256$ versus o logaritmo na base 2 do número de elementos finitos.

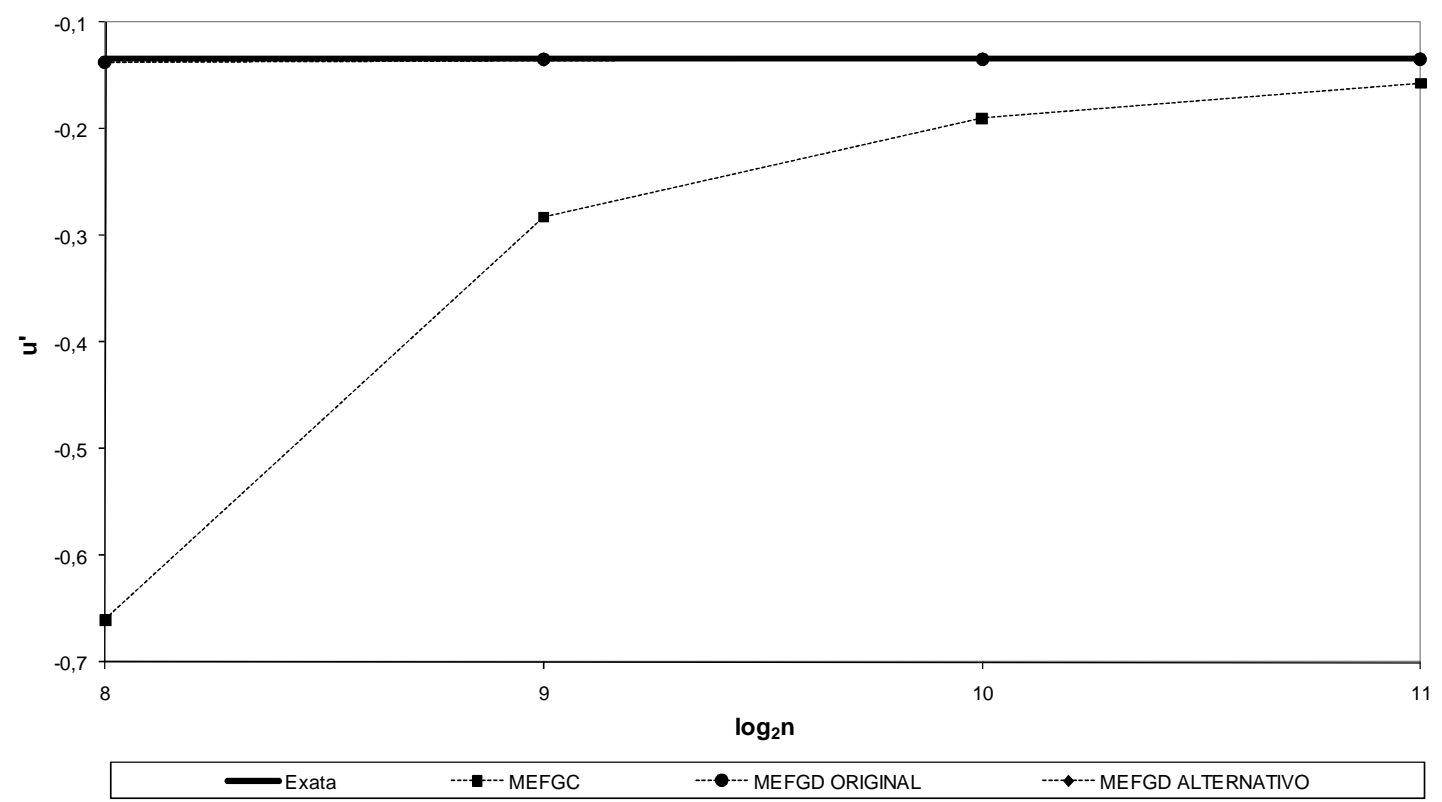

Figura 7.17. Curvas deformação $\mathrm{x} \log _{2} \mathrm{n}$ para ponto na região com auto-intersecção.

A linha cheia horizontal na Fig. 7.17 corresponde ao valor exato desta deformação e as 
outras linhas correspondem aos valores aproximados obtidos com o MEFGC, MEFGD original e MEFGD alternativo. Observa-se da Fig. 7.17 que a curva obtida com a formulação do MEFGC indica tendência de convergência assintótica para o valor exato de deformação. Observa-se também que ambas as formulações do MEFGD fornecem resultados numéricos que estão de muito bom acordo com o valor exato de deformação e que estes resultados são mais precisos do que os resultados obtidos com o MEFGC. Com o objetivo de melhor visualizar as soluções aproximadas obtidas com ambas as formulações do MEFGD mostra-se na Fig. 7.18 uma ampliação da Fig. 7.17 na vizinhança do valor exato de u'(1/256) .

Observa-se da Fig. 7.18 que aqui também os valores nodais de deformação obtidos com ambas as formulações do MEFGD indicam tendência de convergência assintótica para o valor exato de u'(1/256). Os erros obtidos com a formulação original do MEFGD são menores do que os erros obtidos com a formulação alternativa do MEFGD. Observa-se também que ambas as formulações do MEFGD fornecem resultados numéricos que estão de muito bom acordo com o valor exato de deformação e que estes resultados são mais precisos do que os resultados obtidos com o MEFGC.

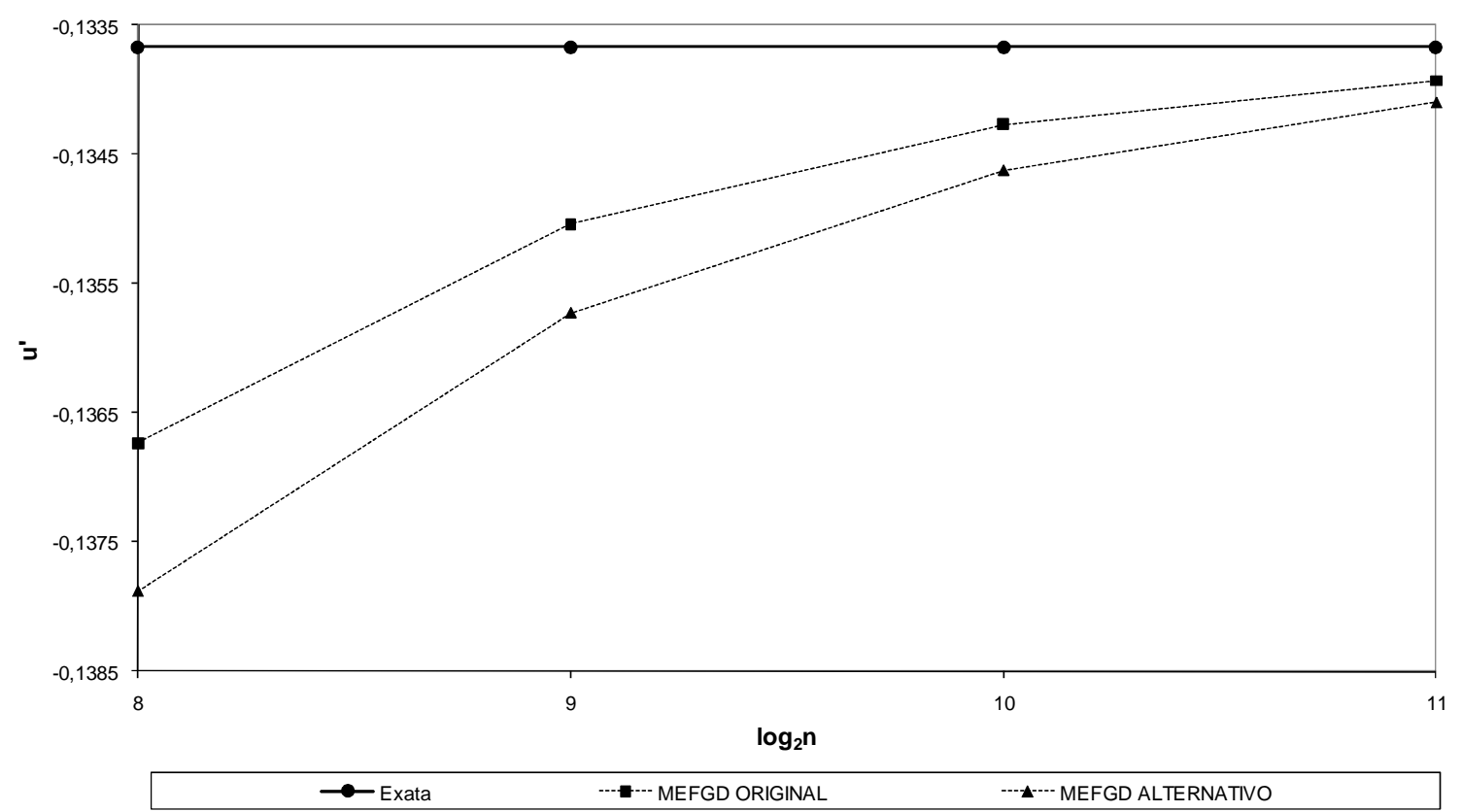

Figura 7.18. Curvas deformação $\mathrm{x} \log _{2} \mathrm{n}$ ampliadas obtidas com MEFGD original e MEFGD alternativo. 


\subsection{OBSERVAÇÕES FINAIS}

Estudou-se o problema da esfera anisotrópica radialmente simétrica submetida à compressão radial uniformemente distribuída sobre a sua superfície externa no contexto da teoria da elasticidade linear clássica. A solução deste problema prediz o fenômeno da autointersecção em uma região próxima ao centro da esfera No contexto de uma teoria de minimização com restrição, este fenômeno é eliminado.

Aplicou-se o Método dos Elementos Finitos de Galerkin Clássico (MEFGC) e duas formulações do Método dos Elementos Finitos de Galerkin Descontínuo (MEFGD) para obter soluções aproximadas para o problema da esfera sem restrição.

Embora o problema seja tridimensional, a consideração de deformações radialmente simétricas reduz o mesmo a um problema unidimensional, cujo domínio é o intervalo $\left(0, \rho_{\mathrm{e}}\right)$. Para a aplicação do MEFGC a discretização deste intervalo foi feita com elementos finitos lineares por partes e contínuos nos nós. Para cada nó associa-se um grau de liberdade de deslocamento. Sendo o intervalo discretizado em $\mathrm{n}$ elementos, o sistema linear resultante possui $n$ equações e $n$ incógnitas.

Para a primeira formulação do MEFGD, em que se aproximam o campo de 
deslocamento e de deformação simultaneamente, a discretização do intervalo $\left(0, \rho_{\mathrm{e}}\right)$ foi feita com elementos finitos lineares por partes e descontínuos nos nós. Sobre cada nó do interior do intervalo associam-se dois graus de liberdade em deslocamento, que correspondem aos valores do deslocamento pela direita e pela esquerda do nó. Similarmente, associam-se dois graus de liberdade para a derivada do deslocamento, resultando em quatro graus de liberdade por nó no interior do intervalo $\left(0, \rho_{\mathrm{e}}\right)$. Sobre as extremidades do intervalo associam-se dois graus de liberdade que correspondem aos valores do deslocamento e de sua derivada pelo lado interior do intervalo. Sendo o intervalo discretizado em $\mathrm{n}$ elementos, o sistema linear resultantes possui $4 n$ equações e $4 n$ incógnitas.

A partir da análise dos erros obtidos para malhas uniformes com 2, 4, 8, 16, 32, 64, 128, 256, 512, 1024 e 2048 elementos para ambos os métodos, MEFGC e formulação original do MEFGD, pode-se observar que à medida que se refina a malha de elementos finitos, os erros diminuem, sendo que os erros relacionados à formulação original do MEFGD são menores que os erros relacionados ao MEFGC. Desta forma, embora a formulação original do MEFGD seja mais custosa do ponto de vista computacional, pois envolve um número maior de incógnitas, os melhores resultados obtidos em comparação ao MEFGC justificam a sua aplicação.

A segunda formulação do MEFGD aplicada ao problema da esfera consiste, igualmente a primeira formulação, em introduzir a equação diferencial $q=u^{\prime}$ que neste caso, não é integrada por partes. O sistema de equações resultante fornece, após a inversão, apenas os valores de $\mathrm{u}_{\mathrm{h}}$. Os valores de $\mathrm{q}_{\mathrm{h}}$ são obtidos por pós-processamento a partir dos valores de $\mathrm{u}_{\mathrm{h}} \cdot$

Na discretização do intervalo $\left(0, \rho_{\mathrm{e}}\right)$ para aplicação desta formulação associa-se a cada nó do interior do intervalo apenas os dois graus de liberdade em deslocamento, que 
correspondem aos valores do deslocamento pela direita e pela esquerda do nó. Sobre as extremidades do intervalo associa-se apenas um grau de liberdade que corresponde ao valor do deslocamento pelo lado interior do intervalo. Para uma discretização com n elementos, o sistema linear resultantes possui $2 \mathrm{n}$ equações e $2 \mathrm{n}$ incógnitas.

Observa-se que esta formulação alternativa reduz o número de equações do sistema em comparação à formulação original do MEFGD e, consequentemente, o custo computacional. A partir da análise dos erros obtidos para malhas uniformes com 2, 4, 8, 16, 32, 64, 128, 256, 512, 1024 e 2048 elementos para ambas as formulação do MEFGD, pode-se observar que a formulação alternativa apresenta erros um pouco maiores do que a formulação original do MEFGD, mas ainda assim muito inferiores aos erros obtidos com o MEFGC.

Analisou-se a convergência dos resultados numéricos obtidos para um ponto pertencente à região onde ocorre a auto-intersecção e para um ponto pertencente à região onde não ocorre a auto-interseção da matéria. A partir dos resultados obtidos para o ponto onde não ocorre a auto-intersecção pode-se observar que, para o problema da esfera anisotrópica radialmente simétrica sob compressão radial uniformemente distribuída, o MEFGC fornece resultados que apresentam convergência monótona conforme predito pela literatura (SORIANO, 2003). Os resultados obtidos com o MEFGD permitem observar que com apenas dois elementos finitos a solução numérica correspondente está muito próxima da solução exata do problema. A análise de convergência de um ponto pertencente à região onde ocorre a auto-interseção permite observar, que para problema da esfera aqui estudado, o MEFGC apresenta convergência monótona e o MEFGD convergência não-monótona.

As aproximações obtidas para o campo de deslocamento e para o campo de deformação infinitesimal com ambas as formulações do MEFGD são muito melhores que as aproximações obtidas com o MEFGC.

Por fim, destaca-se a vantagem adicional do MEFGD em relação ao MEFGC que 
possibilita obter diretamente o campo de deformação do problema estudado sem perder a ordem de aproximação como no caso do MEFGC. 


\section{REFERÊNCIAS BIBLIOGRÁFICAS}

AGUIAR, A. R. A Numerical Treatment of Material Overlapping in Elasticity. In: XXV Iberian Latin American Congress on Computational Methods in Engineering, CILAMCE 2004, Recife, 2004.

AGUIAR, A. R. An exterior penalty method to prevent material overlapping in plane elasticity. In: XXVII Iberian Latin American Congress on Computational Methods in Engineering CILAMCE 2006, Belém, 2006a.

AGUIAR, A. R. Local and global injective solutions of the rotationally symmetric sphere problem. Journal of Elasticity. Vol. 84: 99-129, $2006 \mathrm{~b}$.

AGUIAR, A.; FOSDICK, R. A singular problem in incompressible nonlinear elastostatics. Mathematical Models and Methods in Applied Sciences. Vol. 10, No. 8: 11811297, 2000.

AGUIAR, A.; FOSDICK, R. Self-intersection in elasticity. International Journal of Solids and Structures. Vol. 38: 4797-4823, 2001.

AGUIAR, A. R.; SÁNCHEZ, J. A. Investigação da convergência de soluções aproximadas de problemas singulares em elasticidade anisotrópica. In: $13^{\circ}$ Simpósio Internacional de Iniciação Cientifica da Universidade de São Paulo (SICUSP). São Carlos, 2005.

AGUIAR, A. R.; SILVA, W. C. The singular behavior of a rotating disk under external pressure. In: $19^{\text {th }}$ International Congress of Mechanical - COBEM, 2007, Brasília. Proceedings of COBEM, 2007.

AGUIAR, A. R.; FOSDICK, R. L. Self-intersection in an anisotropic solid in the absence of singularity - Part I: Analytical results. In tenth Pan-American Congress of Applied Mechanics - PACAM X, 2008, Cancún. Proceedings of PACAM X, 2008.

AGUIAR, A. R.; FOSDICK, R. L.; SÁNCHEZ, J. A. G. Anisotropic solid in the absence of singularity - Part II: Computational Results. In: Tenth Pan-American Congress of Applied Mechanics - PACAM X, 2008, Cancún. Proceedings of PACAM X, 2008.

ANTMAN, S. S.; NEGRÓN-MARRERO, P. V. The remarkable nature of radially symmetric equilibrium states of aelotropic nonlinearly elastic bodies. Journal of Elasticity. Vol. 18: 131-164, 1987. 
ARAVAS, N.; SHARMA, M. An elastoplastic analysis of the interface crack with contact zones. Journal of Mechanics and Physical Solids. Vol. 39, No. 3: 311-344,1991.

ARNOLD, D. N. An interior penalty procedures for elliptic and parabolic Galerkin method, Lectures Notes in Physics 58, Springer-Verlag, Berlin, 1976.

ARNOLD, D. N.; BREZZI, F.; COCKBURN, B.; MARINI, D. Discontinuous Galerkin methods for elliptic problems. In Discontinuous Galerkin Methods. Theory, Computation and Applications, B. Cockburn, G. E. Karniadakis, and C-W. Shu, eds., Lecture Notes in Comput. Sci. Engrg. Springer Verlag, New York. Vol. 11: 89-101, 2000.

ARNOLD, D. N.; BREZZI, F.; COCKBURN, B.; MARINI, D. Unified analysis of discontinuous Galerkin methods for elliptic problems. SIAM, J. Numer. Anal. Vol. 39, No. 5: 1749-1779, 2002.

ATKINSON, C. On stress singularities and interfaces in linear elastic fracture mechanics. Internacional Journal of Fracture. Vol. 13, No. 6: 807-820, 1977.

BABUSKA, I.; BAUMANN, C. E.; ODEN, J. T. A discontinuous hp finite element method for diffusion problems: 1-D analysis. An international Journal Computer \& mathematics with applications. Vol. 37: 103-122, 1999.

BAKER, G. A. Finite element methods for elliptic equations using nonconforming elements. SIAM J. Numer. Anal. Vol. 19: 45-59, 1977.

BASSI, F.; REBAY, S. A high-order accurate discontinuous finite element method for the numerical solution of the compressible Navier-Stokes equations. J. Comput. Phys. Vol. 131: 267-279, 1997.

BAUMAN, C. E; ODEN, J. T. A discontinuous hp finite element method for convectiondiffusion problems. Comput. Methods Appl. Mech. Engrg. Vol. 175: 311-341, 1999.

BÖSING, P. R. Método de Galerkin Descontínuo para equações elípticas de alta ordem. 2006. 119 f. Tese (Doutorado) - Instituto de Matemática e Estatística, Universidade de São Paulo, São Paulo, 2006.

BREZZI, F.; MANZINI, G.; MARINI, D.; RUSSO, A. Discontinuous Galerkin approximations for elliptic problems. Numerical Methods Partial Differential Eq. Vol. 16: 365-378, 2000. 
CASTILLO, P.; COCKBURN, B.; PERUGIA, I.; SCHÖTZAU, D. An a priori error analysis of the local discontinuous galerkin method for elliptic problems. SIAM J. Numer. Anal. Vol. 38, No. 5: 1676-1706, 2000.

CASTILLO, P.; COCKBURN, B.; PERUGIA, I.; SCHÖTZAU, D. Local discontinuous Galerkin methods for elliptic problems. Communications in Numerical Methods in Engineering. Vol. 18: 69-75, 2002.

CASTILLO, P. A superconvergence result for discontinuous Galerkin methods applied to elliptic problems. Computer Methods in Applied Mechanics and Engineering. Vol. 192: 4675-4685, 2003.

CASTILLO, P. A review of the Local Discontinuous Galerkin (LDG) method applied to elliptic problems. Applied Numerical Mathematics. Vol. 56: 1307-1313, 2006.

CIARLET, P. G. The finite element method for elliptic problems. New York: North Holland Publishing Company, 1979. 530 p.

COCKBURN, B. Discontinuous Galerkin Methods. ZAMM - Z. Angew. Math. Mech. Vol. 83, No. 11: 731-754, 2003.

COCKBURN, B.; KANSCHAT, G.; PERUGIA, I.; SCHÖTZAU, D. Superconvergence of the local discontinuous Galerkin method for elliptic problems on cartesian grids. SIAM J. Numer. Anal. Vol. 39, No. 1: 264-285, 2001.

COCKBURN, B.; KANSCHAT, G.; SCHÖTZAU, D. A locally conservative LDG method for the incompressible Navier-Stokes equations. American Mathematical Society, 2002.

COCKBURN, B.; KARNIADAKIS, G. E.; SHU, C. The development of discontinuous Galerkin methods. In Discontinuous Galerkin Methods. Theory, Computation and Applications, B. Cockburn, G. E. Karniadakis, and C-W. Shu, eds., Lecture Notes in Comput. Sci. Engrg. Springer Verlag, New York. Vol. 11: 1-48, 2000.

COCKBURN, B.; SHU, C-W. The local discontinuous Galerkin method for timedependent convection-diffusion systems. SIAM J. Numer. Anal. Vol. 35, No. 6: 2440-2463, 1998.

COMNINOU, M. The interface crack. Journal of Applied Mechanics. Vol. 44, No. 4: 631636, 1977. 
DE GIORGI, E. Un esempio di estremali discontinue per un problema variazionale di tipo ellittico. Boll. Unione Mat. Ital. Vol. 4, No. 6: 135-137, 1968.

DELVES, L. M.; HALL, C. A. An implicit matching principle for global element calculations. J. Inst. Math Appl. Vol. 23: 223-234, 1979.

DOUGLAS, J.; DUPONT, T. Interior penalty for elliptic and parabolic Galerkin methods. Lectures Notes in Phys. 58, Springer-Verlag, Berlin, 1976.

DUARTE, A. V. C.; ROCHINHA, F. A.; DO CARMO, E. G. D. Discontinuous finite element formulations applied to cracked elastic domains. Computer methods in applied mechanics and engineering. Vol. 185: 21-36, 2000.

ENGLAND, A. H. A crack between dissimilar media. Journal of Applied Mechanics. Vol. 32, No. 2: 400-402, 1965.

FORTI, T. L. D. O método de Galerkin Descontínuo aplicado a problemas de convecçãodifusão. 2005. 94 f. Dissertação (Mestrado) - Faculdade de Engenharia Civil, Arquitetura e Urbanismo, Universidade Estadual de Campinas, Campinas, 2005.

FOSDICK, R.; ROYER-CARFAGNI, G. The constraint of local injectivity in linear elasticity theory. Proceedings of the Royal Society of London. Vol. 457: 2167-2187, 2001.

FOSDICK, R.; FREDDI F.; ROYER-CAFAGNI, G. Bifurcation instability in linear elasticity with the constraint of local injectivity. Journal of Elasticity. Vol. 90: 99-126, 2008.

GUDI, T.; NATARAJ, N.; PANI, A. K. hp-Discontinuous Galerkin Method for strongly nonlinear elliptic boundary value problems. Numerische Mathematik, 2008.

GURTIN, M. E. An introduction to continuum mechanics. New York: Academic Press, 1981.

JOHNSON, C. Numerical solution of partial differential equation by the finite element method. New York: Cambridge University Press, 1987. 279 p.

KNOWLES, J. K. A nonlinear effect in mode II crack problems. Engng Fracture Mech. Vol. 15, No. 3-4: 469-476, 1981. 
KRINDGES, A. Método de Galerkin Descontínuo de Elementos Finitos para Equações Elípticas. 2004. 83 f. Dissertação (Mestrado) - Centro de Ciências Físicas e Matemáticas, Universidade Federal de Santa Catarina, Florianópolis, 2004.

LEKHNITSKII, S. G. Anisotropic Plates. New York: Gordon \& Breach, 1968.

LeSAINT, P.; RAVIART, P. A. On a finite element method for solving the neutron transport equation. Mathematical aspects of finite elements in partial differential equations (C. de Boor, ed.), Academic Press. 89-145, 1974.

MOZOLEVSKI, I.; BÖSING, P. R. Método de Elementos Finitos de Galerkin descontínuo para equações de Navier-Stokes bidimensionais. TEMA Tend. Mat. Apl. Comput. Vol. 6, No. 1: 101-110, 2005.

MOZOLEVSKI, I.; BÖSING, P. R.; SCHUH, L. I. A. Método de Galerkin Descontínuo com penalização de fluxos para a Equação Reação-difusão. TEMA Tend. Mat. Apl. Comput. Vol. 8, No. 2: 287-297, 2007.

MUSKHELISHVILI, N. I. Some basic problems of the mathematical theory of elasticity. New York: P. Noordoff and Company, 1933.

NASH, J. Continuity of solutions of parabolic and elliptic equations. American Journal of Mathematics. Vol. 80, No. 4: 931-954, 1958.

ODEN, J. T. Mechanics of elastic structures. New York: McGraw-Hill Book Company, 1967. $381 \mathrm{p}$.

PROENÇA, S. P. B. Introdução aos Métodos Numéricos - Notas de Aula. São Carlos, 2007.

REED, W. H.; HILL, T. R. Triangular mesh method for the neutron transport equation. Tech. Report LA-UR-73-479, Los Alamos Scientfic Laboratory, 1973.

RIVIÈRE, B.; WHEELER, M. F.; GIRAULT, V. A priori error estimates for finite element methods based on discontinuous approximation spaces for elliptic problems. SIAM J. Numer. Anal. Vol. 39, No. 3: 902-931, 2001.

SCHNEIDER, R.; XU, Y.; ZHOU, A. An analysis of discontinuous Galerkin methods for elliptic problems. Advances in Computational Mathematics. Vol. 25: 259-286, 2006. 
SILVA, W. C. Relatório de Pesquisa Referente ao Período 06/2007 a 10/2007. Projeto de Iniciação Científica FAPESP, Proc. Nº. 06/50009-0, São Carlos, 2007.

SOKOLNIKOFF, I. S. Mathematical theory of elasticity. $2^{\text {a }}$ edição. New York: McGrawHill, 1956.

SORIANO, H. L. Método de Elementos Finitos em Análise de Estruturas. São Paulo: Editora da Universidade de São Paulo, 2003. 580 p.

TARN, J. Stress singularity in an elastic cylinder of cylindrically anisotropic materials. Journal of Elasticity. Vol. 69: 1-13, 2002.

TAUCHERT, T. R. Energy principles in structural mechanics. New York: McGraw-Hill, 1974.

TIMOSHENKO, S. P; GOODIER, J. N. Teoria da elasticidade. $3^{\text {a }}$ edição, Rio de Janeiro: Ed. Guanabara Dois, 1980.

TING, T.C.T. Anisotropic Elasticity. New York: Oxford University Press, 1996.

TING, T.C.T. The Remarkable Nature of Radially Symmetric Deformation of Spherically Uniform Linear Anisotropic Elastic Solids. Journal of Elasticity Vol. 53: 4764, 1999.

VALLIAPPAN, S. Continuum mechanics fundamentals. Rotterdam: Distributed in USA \& Canada by MBS, 1981.

VILLAÇA, S. F.; GARCIA, L. F. T. Introdução à teoria da elasticidade. $4^{\text {a }}$ edição. Rio de Janeiro: COPPE/UFRJ, 2000.

WEINBERGER, H. F. A first course in partial differential equations with complex variables and transform methods. U.S.A: Blaisdell Publishing Company, 1965. 446 p.

WHEELER, M. F. An elliptic collocation-finite element method with interior penalties. SIAM J. Numer. Anal. Vol. 15, No. 1: 152-161, 1978.

WILLIAMS, M. L. The stresses around a fault or crack in dissimilar media. Bulletin of the Seismological Society of America. Vol. 49, No. 2: 199-204, 1959. 